\title{
NONCOMMUTATIVE PRÜFER RINGS AND
} SOME GENERALIZATIONS

\author{
by \\ YIQIANG ZHOU \\ B.Sc., Hunan Normal University, 1981 \\ M.Sc., Beijing Normal University, 1984 \\ A THESIS SUBMITTED IN PARTIAL FULFILLMENT OF \\ THE REQUIREMENTS FOR THE DEGREE OF \\ DOCTOR OF PHILOSOPHY
}

in

THE FACULTY OF GRADUATE STUDIES

(Department of Mathematics)

We accept this thesis as conforming

to the required standard

THE UNIVERSITY OF BRITISH COLUMBIA

April 1993

(C)Yiqiang Zhou, 1993 
In presenting this thesis in partial fulfilment of the requirements for an advanced degree at the University of British Columbia, I agree that the Library shall make it freely available for reference and study. I further agree that permission for extensive copying of this thesis for scholarly purposes may be granted by the head of my department or by his or her representatives. It is understood that copying or publication of this thesis for financial gain shall not be allowed without my written permission.

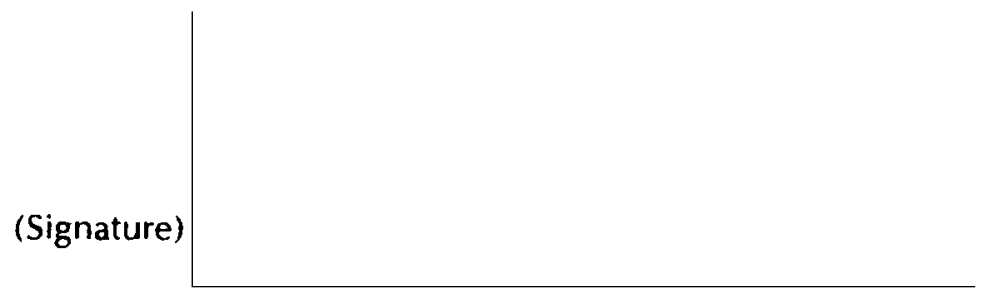

Department of Mathematics

The University of British Columbia

Vancouver, Canada

Date April 30.1993 


\section{Abstract}

Noncommutative Prüfer rings appear naturally when one wants to transfer the known results for rings which arise in algebraic geometry (such as Dedekind, Krull and Prüfer, valuation rings ...) to noncommutative rings. We remove the left-right symmetry condition of the noncommutative Prüfer rings introduced by Alajbegovic and Dubrovin, and introduce three natural generalizations, semi-Prüfer rings, right $w$-semi-Prüfer rings, and right $w$ Prüfer rings. We study the relations between the four concepts, and present the various properties that characterize them. We formulate and prove the basic facts for those rings (decompositions of such rings; Morita invariants of these notions; relations with some other notions). A new module-theoretic characterization of semiprime right Goldie rings is achieved by using the newly-defined concept of strongly compressible modules. The result is used to provide new characterizations of semiprime Goldie (prime right Goldie, or prime Goldie) rings, and right $w$-semi-Prüfer (semi-Prüfer, right $w$-Prüfer, or Prüfer) rings. In particular, the characterization of semiprime Goldie rings of Lopez-Permouth, Rizvi, and Yousif using weakly-injective modules is an easy corollary of our results. We also study modules over noncommutative Prüfer rings. It is shown that a module over a noncommutative Prüfer ring has projective dimension at most one if and only if it is the union of a well-ordered continuous chain of submodules with each factor of the chain a finitely presented cyclic module. The result is used to present a characterization of divisible modules with projective dimension at most one over noncommutative Prüfer rings, which generalizes a known result of L.Fuchs. 


\section{Contents}

$\begin{array}{ll}\text { Abstract } & \text { ii }\end{array}$

Table of contents

Notations iv

Acknowledgements vii

$\begin{array}{ll}\text { Introduction } & 1\end{array}$

$\begin{array}{lll}1 \text { The Preliminaries } & 10\end{array}$

2 Noncommutative Prüfer rings and some generalizations 10

2.1 Definitions and properties ............ 11

2.2 A structure theorem and further properties of right $w$-semi-Prüfer rings . . . . . . . . . . . 21

2.3 Prüfer rings and semi-Prüfer rings . . . . . . . . 27

3 Strongly compressible modules $\quad 40$

3.1 New characterizations of semiprime right Goldie rings 41

3.2 Some applications . . . . . . . . . . . . . 47

4 Modules over Prüfer rings $\quad 54$

4.1 Modules of projective dimension at most one . . . . . 54

4.2 Divisible modules of projective dimension at most one 67

$\begin{array}{lr}\text { References } & \mathbf{7 9}\end{array}$ 


\section{Notations}

Notations in this manuscript are fairly standard, and may be found in most graduate level texts on Algebra and Ring Theory. To keep the reader on track, we will introduce them as required. The following two books are our main references:

1) Rings and Categories of Modules by F.W. Anderson and K.R. Fuller, and

2) An Introduction to Noncommutative Noetherian Rings by K.R. Goodearl and R.B. Warfield. Jr.

We will feel free to use the results in the two books whenever we have such a demand.

Throughout this manuscript, a ring $R$ will mean a nonzero associative noncommutative ring with an identity. And all modules are unitary. The notation $M_{R}$ (or ${ }_{R} M$ ) indicates that $M$ is a right (or left) module over a ring $R$. Given a module $M_{R}$, we will denote by $E\left(M_{R}\right)$ the injective hull of the module $M_{R}$. For a subset $X$ of a right $R$-module $M$, the annihilator right ideal of $X$ in $R$ is denoted by $X^{\perp}$, i.e., $X^{\perp}=\{r \in R: x r=0$ for all $x \in X\}$. Similarly, for a left $R$-module ${ }_{R} N$ and a subset $Y$ of $N$, we denote the annihilator left ideal of $Y$ in $R$ by ${ }^{\perp} Y$. In particular, we write $x^{\perp}$ (or ${ }^{\perp} y$ ) to indicate $\{x\}^{\perp}$ (or $\left.{ }^{\perp}\{y\}\right)$.

Mod- $R \quad$ the category of all right $R$-modules

$R$-Mod the category of all left $R$-modules

$\subset \quad$ proper inclusion 
$\mathbf{N}$

$\mathbf{Z}$

$\operatorname{End}(M) \quad$ the ring of all module endomorphisms of a module $M$

$Z\left(M_{R}\right) \quad$ the singular submodule of a module $M_{R}$

$\tau(M) \quad$ the torsion submodule of a module $M$

$T(M) \quad$ the trace ideal of a module $M$

$M^{*} \quad$ the dual module of a module $M$

$\operatorname{dim}(M) \quad$ the Goldie dimension of a module $M$

$P d(M) \quad$ the projective dimension of a module $M$

$M^{(I)} \quad$ the direct sum of $I$ copies of $M$

$M^{(n)} \quad$ the direct sum of $n$ copies of $M$

$M_{n}(R) \quad$ the $n$ by $n$ matrix ring over a ring $R$

$\operatorname{Rad}(R) \quad$ the Jacobson radical of a ring $R$

$\mathcal{C}_{R}(0) \quad$ the set of all regular elements of $R$

$Q_{c l}^{r}(R) \quad$ the classical right quotient ring of a ring $R$ (if it exists)

$Q_{c l}^{l}(R) \quad$ the classical left quotient ring of a ring $R$ (if it exists)

$Q_{c l}(R) \quad$ the classical quotient ring of a ring $R$ (if it exists) 
ACC the ascending chain condition

$\otimes$

tensor product

Ext the extension functor 


\section{Acknowledgements}

I would like to express my deep thanks to my thesis supervisor, Dr. Stanley S.Page, for his invaluable advice and kindly encouragement throughout past few years. The many many hours of discussion with him on ring theory made my stay at the University of British Columbia a most pleasurable experience of study of my life.

I would especially like to thank my wife, Hongwa, for her support, love, and understanding. 


\section{Introduction}

Prüfer domains form an important and much-studied class of integral domains in Commutative Algebra. With Dedekind domains, valuation domains and Krull domains, they constitute the main objects of study in the Multiplicative Theory of Ideals. The importance of the class of Prüfer domains lies mainly in: 1) Prüfer domains have an origin in Algebraic Number Theory. The rings of integers of finite algebraic number fields, which are the main objects of study in Algebraic Number Theory, are Prüfer domains. 2) Prüfer domains have tight connections with Dedekind domains and valuation domains. In fact, the class of Dedekind domains is precisely the class of Noetherian Prüfer domains; and a Prüfer domain can be characterized as an integral domain such that the localization of it at any prime (or maximal) ideal is a valuation domain. 3) The lattice of all ideals of a Prüfer domain possesses many beautiful arithmetics. For example, an integral domain is a Prüfer domain if and only if $A(B \cap C)=A B \cap A C$ for all ideals $A, B, C$ of $R$ if and only if $A \cap(B+C)=A \cap B+A \cap C$ for all ideals $A, B, C$ of $R$.

In the past twenty years, the study of the noncommutative analogues of Dedekind domains, valuation domains and Krull domains has been a fascinating area of study in ring theory. And many results have been obtained on the various generalizations of them to noncommutative cases, e.g., Asano orders, Dedekind prime rings, hereditary Noetherian prime rings, chain rings, Dubrovin valuation rings, Chamerie Krull rings, Marubaynshi Krull rings, $\Omega$ Krull rings, and others. It is from the abundance of the study of these objects and the close relations between Prüfer domains, Dedekind domains, valua- 
tion domains and Krull domains that one sees the need for an introduction of noncommutative analogues of Prüfer domains.

Let $R$ be an integral domain with field of quotients $Q$. An $R$-submodule $I$ of $Q$ is said to be a fractional ideal of $R$ if $d I \subseteq R$ for some $0 \neq d \in R$. For each fractional ideal $I$ of $R$, define $I^{-}=\{q \in Q: q I \subseteq R\}$. Then $I$ is said to be invertible if $I^{-} I=R$. We call an integral domain $R$ a Prüfer domain if every nonzero finitely generated (f.g. for short) ideal of $R$ is invertible. Prüfer domains can be characterized as any commutative rings with the property that each nonzero f.g. ideal is a progenerator, or is projective, or is a generator. This large selection of attributes suggests many possible generalizations to noncommutative cases, and at the same time raises the difficulty of the best choice among such numerous generalizations. In 1990, Alajbegovic and Dubrovin defined a noncommutative (right) Prüfer ring as a prime Goldie ring such that $I^{-1} I=R$ and $I I^{-1}=O_{l}(I)$ for every f.g. fractional right ideal $I$ of $R$, where $O_{l}(I)=\left\{q \in Q_{c l}(I): q I \subseteq I\right\}$ and $I^{-1}=\left\{q \in Q_{c l}(R): I q I \subseteq I\right\}$. Among the properties of noncommutative Prüfer rings, they show that the concept of a noncommutative Prüfer ring is a left-right symmetric concept; the notion is a Morita invariant, and every noncommutative Prüfer ring is Morita equivalent to a (noncommutative) Prüfer domain. They also note that the class of noncommutative Prüfer rings contains the classes of prime Dedekind rings, Dubrovin valuation rings, and commutative Prüfer domains.

The present manuscript is devoted to continuing the study of noncommutative Prüfer rings. We first observe that a noncommutative Prüfer ring can be characterized as a prime Goldie ring $R$ such that every nonzero f.g. 
submodule of a progenerator of Mod- $R$ is a progenerator. The nature of the characterization brought our interests to noncommutative Prüfer rings. We note that the generalized discrete valuation ring of H.H.Brungs (see [5]) and the skew polynomial rings (see the example in $§ 2.1$, of Chapter 2) provide examples of prime right (but not left) Goldie rings satisfying the same property as above. The observation leads us to remove the left-right symmetric condition of noncommutative Prüfer rings and to consider more general definitions where the conditions of being a prime ring, being a Goldie ring are replaced by a semiprime ring, by a right Goldie ring respectively.

The manuscript is organized into four chapters. Chapter 1 summarizes certain basic concepts and theorems in ring theory which are needed in the sequel. Since they are all well-known and easy to find for reference, the proofs of most of them are omitted.

In Chapter 2, we introduce three generalizations of noncommutative Prüfer rings, semi-Prüfer rings, right $w$-semi-Prüfer rings, and right $w$-Prüfer rings. We study the relations between the four concepts, and present the various properties that characterize them. We formulate and prove the basic facts for those rings (decompositions of such rings; Morita invariants of these notions; relations with some other notions).

In Chapter 3, a new module-theoretic characterization of semiprime right Goldie rings is achieved by using the newly-defined concept of strongly compressible modules. The result is used to provide new characterizations of semiprime Goldie (prime right Goldie, or prime Goldie) rings, and right $w$ semi-Prüfer (semi-Prüfer, right $w$-Prüfer, or Prüfer) rings. In particular, the characterization of semiprime Goldie rings of Lopez-Permouth, Rizvi, and 
Yousif using weakly-injective modules is an easy corollary of our results.

Chapter 4 is provided to study modules over noncommutative Prüfer rings. The study is motivated by the work of L.Fuchs on modules over valuation (or Prüfer) domains (see [13]). We give a characterization of modules of projective dimension at most one over noncommutative Prüfer rings, and present a structure theorem of divisible modules with projective dimension at most one over noncommutative Prüfer rings, which generalizes a known result of L.Fuchs. 


\section{The Preliminaries}

This chapter is provided to review a number of basic concepts and some important results from ring theory, which will be used throughout the sequel. The proofs for most results are omitted, since they can be found in the standard texts in ring theory, such as [2] and [18].

\section{Essential extensions and singular submodules}

An essential submodule of a module $M$ is any submodule $N$ which has nonzero intersection with every nonzero submodule of $M$. We write $N \leq_{e} M$ to denote this situation, and we also say that $M$ is an essential extension of $N$.

Proposition 1.1 (a) Let $N$ be a submodule of a module $M$, and let $f$ : $P \longrightarrow M$ be a homomorphism. If $N \leq_{e} M$, then $f^{-1}(N) \leq_{e} P$.

(b) Let $N$ be a submodule of a module $M$, and $P$ a submodule of $M$ which is maximal with respect to the property $P \cap N=0$. Then $N \oplus P \leq_{e} M$ and $(N \oplus P) / P \leq_{e} M / P$.

The singular submodule of a module $M_{R}$ is defined by $Z\left(M_{R}\right)=\{x \in M$ : $\left.x^{\perp} \leq_{e} R_{R}\right\}$. Since $Z\left(M_{R}\right)$ is a fully invariant submodule of $M$, the right singular ideal $Z\left(R_{R}\right)$ is an ideal of $R$. If $Z\left(M_{R}\right)=0$ then $M$ is called a nonsingular module. The ring $R$ is called a right non-singular ring if $Z\left(R_{R}\right)=0$. A right and left non-singular ring is called a non-singular ring. 


\section{Orders and quotient rings}

A regular element in a ring $R$ is any non-zero-divisor, i.e., any element $x \in R$ such that $x^{\perp}={ }^{\perp} x=0$. We will denote by $\mathcal{C}_{R}(0)$ the set of all regular elements of $R$.

Definition 1.1 Let $Q$ be a ring. A right order in $Q$ is any subring $R \subseteq Q$ such that

(a) every regular element of $R$ is invertible in $Q$;

(b) every element of $Q$ has the form $a b^{-1}$ for some $a \in R$ and some $b \in \mathcal{C}_{R}(0)$.

A left order is defined analogously, and a left and right order is called an order.

Definition 1.2 Let $R$ be a ring. A classical right quotient ring, denoted by $Q_{c l}^{r}(R)$ if it exists, is any overring $Q \supseteq R$ such that $R$ is a right order in $Q$. A classical left quotient ring is defined analogously, and a classical left and right quotient ring is called a classical quotient ring.

In Asano [3] it is shown that $Q_{c l}^{r}(R)$ exists if and only if $R$ satisfies the right Ore condition, i.e., for any $a \in R$ and any $c \in \mathcal{C}_{R}(0)$ there exist $b \in R$ and $d \in \mathcal{C}_{R}(0)$ such that $a d=c b$ (a right (or left) Ore ring is any ring satisfying the right (or left) Ore condition). When both $Q_{c l}^{r}(R)$ and $Q_{c l}^{l}(R)$ exist, we have $Q_{c l}^{r}(R) \cong Q_{c l}^{l}(R)$. This occurs only when $R$ is an order. We will denote by $Q_{c l}(R)$ the classical quotient ring of $R$ (if it exists). Another basic fact is that the classical right quotient ring (if it exists) is unique, up to isomorphism (see [18, Cor.9.5, P146]). 
Lemma 1.1 Let $R$ be a right order with $Q=Q_{c l}^{r}(R)$ and let $S$ be an overring of $R$, i.e., $R \subseteq S \subseteq Q$. If $I$ is a right $S$-submodule of $Q$ such that I contains a regular element of $R$, then $\operatorname{Hom}_{S}\left(I_{S}, S_{S}\right)=\left\{\sigma_{q}: q \in Q, q I \subseteq S\right\}$, where $\sigma_{q}: I \longrightarrow S$ is a S-homomorphism defined by $\sigma_{q}(x)=q x$.

Proof. For each $q \in Q$ with $q I \subseteq S$, it is easy to see that $\sigma_{q}$ is a $S$ homomorphism. Suppose $\phi: I \longrightarrow S$ is a $S$-homomorphism. Let $s \in I$ be a regular element of $R$. For each $x \in I$ there exists a regular element $t$ of $R$ such that $x t \in R$. Now, by the right Ore condition, there exist $a \in R$ and $u \in \mathcal{C}_{R}(0)$ such that $s a=x t u$. Then $\phi(x) t u=\phi(x t u)=\phi(s a)=$ $\phi(s) a=\phi(s) s^{-1} s a=\phi(s) s^{-1} x t u$, which implies $\phi(x)=\phi(s) s^{-1} x=\sigma_{q}(x)$ with $q=\phi(s) s^{-1}$ satisfying $q I \subseteq S$.

\section{Goldie rings and Goldie Theorems}

A right annihilator in a ring $R$ is any right ideal $I$ of $R$ such that $I=X^{\perp}$ for some $X \subseteq R$. Left annihilators are defined in a similar way. Note that a right ideal $I$ is a right annihilator if and only if $I=\left({ }^{\perp} I\right)^{\perp}$.

A module $M_{R}$ is called finite-dimensional (or in other words, $M_{R}$ has finite Goldie dimension) if $M$ does not contain an infinite direct sum of nonzero submodules. In this case, there exists a nonnegative integer $n$ such that $M$ contains a direct sum of $n$ nonzero submodules, but no direct sum of $n+1$ nonzero submodules. Such an $n$ is uniquely determined by $M$. We shall call this integer the Goldie dimension of $M_{R}$, and denote it by $\operatorname{dim}\left(M_{R}\right)$.

Definition 1.3 $A$ right Goldie ring is any ring $R$ such that $R_{R}$ is finitedimensional and $R$ has $A C C$ on right annihilators. 
The Preliminaries

Proposition 1.2 [Goldie]. Let $R$ be a semiprime right Goldie ring, and let $I$ be a right ideal of $R$. Then $I$ is an essential right ideal if and only if $I$ contains a regular element.

Theorem 1.1 [Goldie]. Let $R$ be a ring.

(a) $R$ is a right order in a semi-simple ring if and only if $R$ is a semiprime right Goldie ring;

(b) $R$ is a right order in a simple Artinian ring if and only if $R$ is a prime right Goldie ring.

Theorem 1.2 Let $R$ be semiprime. Then $R$ is a right Goldie ring if and only if $Z\left(R_{R}\right)=0$, and $R_{R}$ is finite-dimensional.

\section{Torsion modules and torsionfree modules}

Given a module $M_{R}$, let $\tau(M)=\left\{x \in M: x r=0\right.$ for some $\left.r \in \mathcal{C}_{R}(0)\right\}$. If $R$ is a right order, then $\tau(M)$ is a submodule of $M$. In fact, for $x, y \in \tau(M)$ and $r \in R$, we have $x s=0=y t$ for some $s, t \in \mathcal{C}_{R}(0)$. By the right Ore condition, there exist $c, d \in \mathcal{C}_{R}(0)$, and $a, b \in R$, such that $s c=t a$ and $a d=r b$. Then we have $(x-y) s c=x s c-y t d=0$ and $(x a) d=x r b=0$. When $\tau(M)$ is a submodule, it is called the torsion submodule of $M$. If $\tau(M)=M$, then $M$ is called a torsion module, and if $\tau(M)=0$, then $M$ is called a torsionfree module. Clearly $M / \tau(M)$ is torsionfree for every module $M_{R}$. If $R$ is a semiprime Goldie ring, then, because of Proposition 1.2, $Z\left(M_{R}\right)=\tau\left(M_{R}\right)$ for every module $M_{R}$. 
The Preliminaries

Theorem 1.3 [Gentile, Levy]. If $R$ is a semiprime Goldie ring and $M$ is a f.g. torsionfree right $R$-module, then $M$ can be embedded in a f.g. free right $R$-module.

\section{Morita equivalences}

Given a right $R$-module $M$. We let $M^{*}=\operatorname{Hom}(M, R)$. The trace of $M$, written $T\left(M_{R}\right)$, is defined by $T(M)=\Sigma\left\{f(M): f \in M^{*}\right\}$. It is clear that $T(M)$ is an ideal of $R$. We now call a right $R$-module $X$ a generator of the category Mod- $R$ if the trace ideal $T(M)=R$. The concept of generator plays a central role in the study of equivalences between categories of modules. The following proposition gives a number of important characterizations of generators.

Proposition 1.3 The following are equivalent for a module $X \in \operatorname{Mod}-R$ :

(a) $X$ is a generator;

(b) For every $M \in M o d-R$, there is an index set $I$ such that $M$ is a homomorphism image of $X^{(I)}$, where $X^{(I)}$ is the direct sum of I copies of $X$;

(c) There exists an $n$ such that $R$ is a homomorphism image of $X^{(n)}$.

A module $P$ is called projective if given an epimorphism $p: M \longrightarrow N$, then any homomorphism $f: P \longrightarrow N$ can be factored as $f=p \circ g$ for some $g: P \longrightarrow N$. It is well-known that a module $P_{R}$ is projective if and only if $P$ is a direct summand of a free module if and only if any short exact sequence $0 \rightarrow M \rightarrow N \rightarrow P \rightarrow 0$ splits. A very useful criterion for projectivity is the following proposition which is often called the "dual basis lemma" for projective modules. 
Proposition 1.4 $A R$-module $P_{R}$ is projective if and only if there exist a set $\left\{x_{\alpha}: \alpha \in I\right\}$ of elements in $P$ and a set $\left\{f_{\alpha}: \alpha \in I\right\}$ of elements in $P^{*}=$ Hom $(P, R)$, such that for any $x \in P, f_{\alpha}(x)=0$ for all but finite number of the $f_{\alpha}$, and $x=\Sigma_{\alpha \in I} x_{\alpha} f_{\alpha}(x)$.

A module $X$ is a progenerator of $\operatorname{Mod}-R$ if and only if $X$ is f.g. projective and $X$ is a generator of $\operatorname{Mod}-R$.

Definition 1.4 Let $F, G$ be functors from Mod-R to Mod-S. We say there is a natural isomorphism from $F$ to $G$, written $F \cong G$, if there exists a map that assigns to every module $M \in \operatorname{Mod}-R$ an isomorphism $\phi_{M} \in$ $\operatorname{Hom}_{S}(F(M), G(M))$ such that for any $M, N \in \operatorname{Mod}-R$ and any $f \in$ $\operatorname{Hom}_{R}(M, N)$ the following diagram:

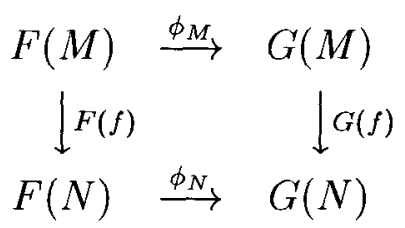

is commutative.

Definition 1.5 Two rings $R$ and $S$ are said to be Morita equivalent, written $R \sim S$, if there exist functors $F:$ Mod- $R \longrightarrow \operatorname{Mod}-S$ and $G: \operatorname{Mod}-S \longrightarrow$ Mod-R such that $G F \cong 1_{M o d-R}, F G \cong 1_{M o d-S}$. In this case, $F$ is called a Morita equivalence and $G$ an inverse equivalence between Mod-R and Mod-S. Any two rings which are isomorphic are of course Morita equivalent. The fact that $R \sim M_{n}(R)$, where $M_{n}(R)$ is the ring of $n$ by $n$ matrices with entries in $R$, shows simply that a noncommutative ring may be Morita equivalent to a commutative ring. 
Theorem 1.4 For two rings $R$ and $S$, then $R \sim S$ if and only if $S \cong$ $\operatorname{End}(M)$ for some progenerator $M$ of $M o d-R$. And in this case, $H_{R} m_{R}(M,-)$ : $N_{R} \longmapsto \operatorname{Hom}_{R}\left({ }_{S} M_{R}, N_{R}\right)$ defines a Morita equivalence between Mod-R and Mod-S with inverse equivalence $-\otimes_{S} M: P_{S} \longmapsto P \otimes_{S} M$.

Theorem 1.5 For two rings $R$ and $S$, then $R \sim S$ if and only if $S \cong$ $e M_{n}(R)$ e for some $n$ and some idempotent e of $M_{n}(R)$ with $M_{n}(R) e M_{n}(R)=$ $M_{n}(R)$.

Any ring property which is preserved under Morita equivalence is called a Morita invariant. For example, being a semiprime right Goldie ring is a Morita invariant because any ring Morita equivalent to a semiprime right Goldie ring is semiprime right Goldie [29, Propo.5.10].

\section{Semihereditary rings}

A ring is right (or left) semihereditary if every f.g. right (or left) ideal is projective. A right and left semihereditary ring is called a semihereditary ring. An example of a ring which is right but not left semihereditary was given by Chase [6]. In the following, we introduce a theorem of Small which presents certain classes of rings for which right semihereditary implies left semihereditary.

Theorem 1.6 [Small]. Let $R$ be a ring in which every principal right ideal is projective and in which there is no infinite set of orthogonal idempotents. Then every right and every left annihilator is generated by an idempotent. In particular, every principal left ideal is projective. 
Proof. Suppose $0 \neq T=S^{\perp}$. If $s \in S$, then $T \subseteq s^{\perp}$. Thus, $T \subseteq h R$ where $h$ is an idempotent. Now let $L$ be an arbitrary (nonzero) left annihilator. $L^{\perp} \subseteq g R$ where $g^{2}=g$. But then $L={ }^{\perp}\left(L^{\perp}\right) \supseteq^{\perp}(g R)=R(1-g)$. Hence, any left annihilator $L$ contains a nontrivial idempotent. By [2, Ex. 10.11], we can choose an idempotent $e \in L$ such that ${ }^{\perp} e$ is minimal amongst the left annihilators of idempotents in $L$. We claim ${ }^{\perp} e \cap L=0$. Suppose not. Then ${ }^{\perp} e \cap L$ is a nonzero left annihilator which contains an idempotent $f \neq 0$. Now $e^{*}=e+f-e f$ is an idempotent in $L$. Since $e^{*} e=e, e^{*} \neq 0$ and ${ }^{\perp} e^{*} \subseteq^{\perp} e$. However, $f e=0$ and $f e^{*}=f \neq 0$. Thus, ${ }^{\perp} e^{*} \mathbb{}^{\perp} e$; which contradicts the minimality of ${ }^{\perp} e$. Hence ${ }^{\perp} e \cap L=0$. Now if $x \in L$, then $x-x e \in L$ and $(x-x e) e=0$. Therefore $x-x e=0$ and $L=R e$. Finally, if $K$ is a right annihilator, then ${ }^{\perp} K=R e$ where $e^{2}=e$. But, $K=\left({ }^{\perp} K\right)^{\perp}=(1-e) R$.

Proposition 1.5 A ring $R$ is right (left) semihereditary if and only if $M_{n}(R)$, for all $n$, has principal right (left) ideals projective.

Proof. It is well known that if $R$ is right (left) semihereditary, then so is $M_{n}(R)$.

In the other direction, we must show that any f.g. right ideal, say $I=$ $a_{1} R+\cdots+a_{n} R$, is projective. In $M_{n}(R)$ let $x$ be the matrix $\left(c_{i j}\right)$ where $c_{1 i}=a_{i}$ and all other entries are zero. Then $x M_{n}(R)$ is projective as a right $M_{n}(R)$-module. But, $x M_{n}(R)$ considered as a right $R$-module ( $R$ embedded in $M_{n}(R)$ in the usual way) is isomorphic to $I \oplus \cdots \oplus I$ ( $n$ times). Thus, since $M_{n}(R)$ is $R$-free, $I \oplus \cdots \oplus I$ is $R$-projective and $I$ is $R$-projective.

Combining Theorem 1.6 and Proposition 1.5, we immediately obtain 
Theorem 1.7 [Small]. Suppose $R$ is a ring which is right semihereditary and such that $M_{n}(R)$, for all $n$, does not possess an infinite set of orthogonal idempotents, then $R$ is left semihereditary. 


\section{Noncommutative Prüfer rings and some generalizations}

(Noncommutative) Prüfer rings were introduced and studied by Alajbegovic and Dubrovin [1]. Examples of Prüfer rings include prime Dedekind rings, commutative Prüfer domains and prime Goldie right (or left) Bezout rings (cf.[1]. Examples 1.13 and 1.15). Some important properties of Prüfer rings have been demonstrated in the paper of Alajbegovic and Dubrovin (cf.[1] for details). An observation is that a ring $R$ is a Prüfer ring if and only if $R$ is a prime Goldie ring with the following property (see Proposition 2.1.1):

(P): Every finitely generated essential right ideal of $R$ is a progenerator of Mod- $R$. Replacing 'prime Goldie' by 'semiprime Goldie', 'prime right Goldie', and 'semiprime right Goldie', respectively, in the above condition, we introduce three natural generalizations of Prüfer ring which are to be called (right) semi-Prüfer ring, right $w$-semi-Prüfer ring, and right $w$-Prüfer ring respectively (see section 1 for the precise definitions). The main object of this chapter is to study the relationship between all these rings and to establish various properties of them.

In section 1, we first give the definitions of three generalizations of Prüfer rings. The four concepts, especially their implication relations, are further explained by using a known example. The rest of section 1 is used to present the various properties and characterizations of all these rings. In section 2 , we will present a structure theorem of right $w$-semi-Prüfer rings. " A ring is a right $w$-semi-Prüfer ring if and only if it is a finite direct sum of right 
$w$-Prüfer rings". Section 3 is devoted to studying Prüfer rings and semiPrüfer rings. We will show that the right semi-Prüfer rings are exactly the left semi-Prüfer rings. A structure theorem states that a ring is a semi-Prüfer ring if and only if it is a finite direct sum of Prüfer rings. We will pay special attention to the cases where the Prüfer ring $R$ is a Noetherian, bounded, semiperfect ring respectively. It was proved in [1] that every Prüfer ring is Morita equivalent to a Prüfer domain. We will give a stronger result here which says that every Prüfer ring $R$ can be decomposed as a finite direct sum of uniform submodules such that the endomorphism ring of each of these uniform submodules is a Prüfer domain which is Morita equivalent to $R$. The last result can be used to give a characterization of f.g. torsionfree modules over a semi-Prüfer ring.

\subsection{Definitions and properties}

Let ring $R$ be a right order with $Q=Q_{c l}^{r}(R)$. Given a subset $I$ of $Q$, we set

$$
\begin{array}{cl}
O_{r}(I)=\{q \in Q: I \supseteq I q\} ; & O_{l}(I)=\{q \in Q: I \supseteq q I\} ; \\
{[R: I]_{r}=\{q \in Q: R \supseteq I q\} ;} & {[R: I]_{l}=\{q \in Q: R \supseteq q I\} ;} \\
\text { and } \quad I^{-1}=\{q \in Q: I \supseteq I q I\} .
\end{array}
$$

A submodule $I$ of $Q_{R}$ is called a fractional right ideal of $R$ if $I$ contains a regular element of $Q$, and there exists a regular element $d$ of $Q$ with $R \supseteq d I$. Definition 2.1.1 A semiprime Goldie (semiprime right Goldie or prime right Goldie or prime Goldie) ring $R$ is called a right semi-Prüfer (right $w$-semi-Prüfer or right $w$-Prüfer, or right Prüfer) ring if every finitely generated (f.g. for short) fractional right ideal $I$ of $R$ satisfies: 


$$
I^{-1} I=R, \quad I I^{-1}=O_{l}(I)
$$

The left-sided versions can be defined in a similar way. Clearly every right Prüfer ring is a right semi-Prüfer (right $w$-semi-Prüfer or right $w$-Prüfer) ring, and every right $w$-Prüfer ring is a right $w$-semi-Prüfer ring.

Remark 2.1.1 The definition of a right Prüfer ring is due to Alajbegovic and Dubrovin [1].

Lemma 2.1.1 [32]. If $I$ is a fractional right ideal of a right order $R$, then the following are equivalent:

(a) $I I^{-1}=O_{l}(I)$;

(b) $I$ is a projective right $O_{r}(I)$-module.

Proof. First we note that given a fractional right ideal $I$ of a right order $R$, $O_{r}(I)$ is an overring of $R$ and $I$ is a right $O_{r}(I)$-module.

$(a) \Leftarrow(b)$. By Lemma 1.1, $\operatorname{Hom}_{O_{r}(I)}\left(I, O_{r}(I)\right)=\left\{\sigma_{q}: q \in Q, q I \subseteq\right.$ $\left.O_{r}(I)\right\}=\left\{\sigma_{q}: q \in I^{-1}\right\}$, where for each $q \in I^{-1}, \sigma_{q}: I \longrightarrow O_{r}(I)$ is the $O_{r}(I)$-homomorphism defined by $\sigma_{q}(a)=q a$. Suppose that $I$ is a projective right $O_{r}(I)$-module. Then, by the dual basis lemma, there exist $\left\{a_{\alpha}: \alpha \in\right.$ $X\} \subseteq I$ and $\left\{\sigma_{q_{\alpha}}: \alpha \in X\right\} \subseteq \operatorname{Hom}_{O_{r}(I)}\left(I, O_{r}(I)\right)$ such that for any $a \in$ $I, \sigma_{q_{\alpha}}(a)=q_{\alpha}(a)=0$ for all but a finite number of the $\sigma_{q_{\alpha}}$, and $a=$ $\Sigma_{\alpha \in X} a_{\alpha} \sigma_{q_{\alpha}}(a)$. Choosing $a$ to be regular shows that $q_{\alpha}=0$ for all but a finite number of $\alpha$. Letting $a$ be arbitrary again, we see that $a=\Sigma_{\alpha} a_{\alpha} q_{\alpha} a=$ $\left(\Sigma_{\alpha} a_{\alpha} q_{\alpha}\right) a$. Thus $\Sigma_{\alpha} a_{\alpha} q_{\alpha}=1 \in I I^{-1}$ and hence $I I^{-1}=O_{l}(I)$.

$(a) \Rightarrow(b)$. Suppose that $O_{l}(I)=I I^{-1}$. Then there exist finite sets $\left\{a_{\alpha}\right\} \subseteq I$ and $\left\{q_{\alpha}\right\} \subseteq I^{-1}$ such that $\Sigma_{\alpha} a_{\alpha} q_{\alpha}=1$. Hence $\Sigma_{\alpha} a_{\alpha} q_{\alpha} a=a$. Then 
$\Sigma_{\alpha} a_{\alpha} \sigma_{q_{\alpha}}(a)=a$ with each $\sigma_{q_{\alpha}} \in \operatorname{Hom}_{O_{\tau}(I)}\left(I, O_{r}(I)\right)$. Therefore, by the dual basis lemma, $I$ is a projective right $O_{r}(I)$-module.

Lemma 2.1.2 [1]. If $I$ is a fractional right ideal of a right order $R$, and $L=[R: I]_{l}$, then the following are equivalent:

(a) $L I=R$;

(b) $I_{R}$ is a generator of Mod-R;

(c) $I^{-1} I=R$.

Any of these conditions implies that $O_{r}(I)=R$.

Proof. $(a) \Leftrightarrow(b)$. By definition, $I_{R}$ is a generator of Mod- $R$ if and only if $R=T\left(I_{R}\right)=\Sigma\left\{f(I): f \in \operatorname{Hom}_{R}(I, R)\right\}$. By Lemma 1.1, $\operatorname{Hom}_{R}(I, R)=$ $\left\{\sigma_{q}: q \in L\right\}$. Therefore we have that $I_{R}$ is a generator of Mod- $R$ if and only if $R=\Sigma\left\{\sigma_{q}(I): q \in L\right\}=\Sigma\{q I: q \in L\}=L I$.

Before proving $(b) \Leftrightarrow(c)$, we note the fact that if $K I=R$ for some subset $K$ of $Q$, then $O_{r}(I)=R$. In fact, for $q \in Q$ with $I q \subseteq I$, we have $K I q \subseteq K I$, i.e., $R q \subseteq R$, and thus $q \in R$. In both cases $(a)$ and $(c)$ we therefore can use the equality $O_{r}(I)=R$.

$(c) \Rightarrow(a)$. Now we can take $I^{-1}$ as $K$. Using $(c)$, the inclusion $O_{r}(I) R \subseteq$ $O_{r}(I)$ can be written in an equivalent form $O_{r}(I) I^{-1} I \subseteq O_{r}(I)$. By the definition of $L$ it follows that $O_{r}(I) I^{-1} \subseteq L$, and thus $O_{r}(I) \subseteq L I$, i.e., $R \subseteq L I$. Consequently $R=L I$, and $(a)$ holds.

$(a) \Rightarrow(c)$. This time we can put $K=L$. Also, from the definitions of $I^{-1}, O_{r}(I)$, and $L$ it follows that $L I \subseteq I^{-1} I \subseteq O_{r}(I)$. Hence $R \subseteq I^{-1} I \subseteq R$, i.e., $(c)$ holds. Finally, the remark above shows that either of $(a),(b)$, or $(c)$ implies $R=O_{r}(I)$. 
Proposition 2.1.1 The following are equivalent for a ring $R$ :

(a) $R$ is a right semi-Prüfer (right w-semi-Prüfer, or right w-Prüfer, or right Prüfer) ring;

(b) $R$ is a semiprime Goldie (semiprime right Goldie, or prime right Goldie, or prime Goldie) ring, and every f.g. fractional right ideal of $R$ is a progenerator of Mod- $R$;

(c) $R$ is a semiprime Goldie (semiprime right Goldie, or prime right Goldie, or prime Goldie) ring, and $R$ has property $(\mathbf{P})$.

Proof. We give a proof only for the case where $R$ is a semiprime right Goldie ring.

$(a) \Leftrightarrow(b)$. By Lemma 2.1.1 and Lemma 2.1.2.

$(b) \Rightarrow(c)$. Since $R$ is a semiprime right Goldie ring, every essential right ideal of $R$ contains a regular element of $R$ by Proposition 1.2. Therefore every f.g. essential right ideal of $R$ is a fractional right ideal.

$(c) \Rightarrow(b)$. Let $I$ be a f.g. fractional right ideal of $R$. From the definition of a fractional right ideal, we know that there exist regular elements $c$ and $d$ of $Q$ such that $c \in I$ and $d I \subseteq R$. Then $d c \in d I$, and $d c$ is a regular element of $R$. Hence $d I$ is a f.g. essential right ideal of $R$ by Proposition 1.2. Then (c) implies that $d I$ is a progenerator of Mod- $R$. But we have $I_{R} \cong(d I)_{R}$, so $I_{R}$ is a progenerator of Mod- $R$.

Example 2.1.1 Let $F$ be a field such that there exists an isomorphism $\lambda$ of $F$ onto a proper subfield of $F$. Let $R$ be the abelian group consisting of all polynomials in $x$ with coefficients from $F$, with coefficients written on the right. Define a multiplication in $R$ by using the rule $a x^{n}=x^{n}\left(\lambda^{n} a\right)$ for all 
$a \in F$ and all $n$. Then the ring $R$ is a principal right ideal domain, and $R$ is right Ore but not left Ore [17, Ex.1, P101]. Hence $R$ is right Goldie but not left Goldie. Therefore we have (a) $R$ is a right w-Prüfer ring; $(b) R$ is not a left w-semi-Prüfer ring; $(c) R$ is not a right semi-Prüfer ring.

The example also tells us that being a $w$-Prüfer ring (or a $w$-semi-Prüfer ring) is not a left-right symmetric concept. Since it will be shown that a Prüfer ring or semi-Prüfer ring is left-right symmetric and a ring is a semiPrüfer ring if and only if it is a finite direct sum of Prüfer rings, we have the following implication diagram:

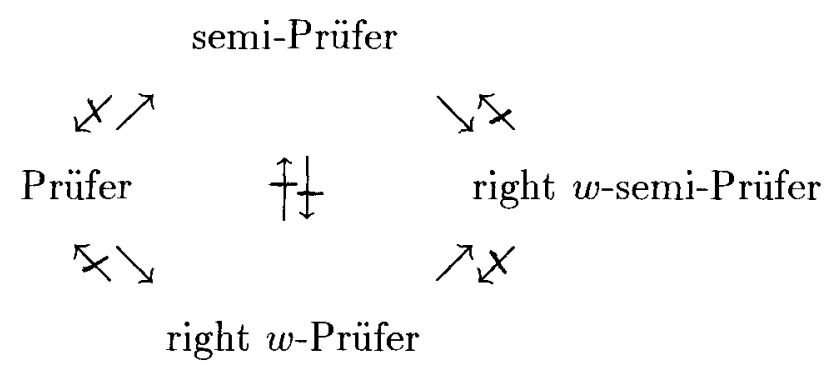

Proposition 2.1.2 Every right w-semi-Prüfer ring is a right and left semihereditary ring.

Proof. Suppose $R$ is a right $w$-semi-Prüfer ring and $I_{R}$ a f.g. right ideal of $R$. We have a right ideal $J$ of $R$ which is maximal with respect to $I \cap J=0$. And $I+J=I \oplus J \leq_{e} R_{R}$. Since $R$ is a semiprime right Goldie ring, $I \oplus J$ contains a regular element $r$ of $R$ by Proposition 1.2. Write $r=a+b, a \in I$ and $b \in J$, and let $K=I \oplus b J$. Then $K$ is a f.g. essential right ideal of $R$. By Proposition 2.1.1 $K_{R}$ is projective, and so is $I_{R}$. We have shown that $R$ is a right semihereditary ring. Because the property of being a semiprime 
right Goldie ring is Morita invariant, $M_{n}(R) \cong \operatorname{End}\left(R^{n}\right)$ is a semiprime right Goldie ring for all $n$, and thus $M_{n}(R)$ does not possess an infinite set of orthogonal idempotents. Hence $R$ is left semihereditary by Theorem 1.7.

Lemma 2.1.3 If a ring $R$ is a right w-semi-Prüfer ring, then $M_{n}(R)$ is a right w-semi-Prüfer ring for every $n$.

Proof. Since the property of being a semiprime right Goldie ring is Morita invariant, $M_{n}(R)$ is a semiprime right Goldie ring. It is also clear that $M_{n}(R)$ is a semihereditary ring because of Proposition 2.1.2 and Theorem 1.7. So it suffices to show that every f.g. essential right ideal $L$ of $M_{n}(R)$ is a generator of $\operatorname{Mod}-M_{n}(R)$. We need some notation: if $A$ is a subset of $R$, set $A[k]=$ $\left\{\left(a_{i j}\right) \in M_{n}(R): a_{i j}=0 \quad \forall i \neq k ; a_{k j} \in A\right\}$. It is easy to see that

$$
L_{M_{n}(R)}=\left(e_{11} L\right)_{M_{n}(R)} \oplus\left(e_{22} L\right)_{M_{n}(R)} \oplus \cdots \oplus\left(e_{n n} L\right)_{M_{n}(R)},
$$

where $e_{k k}$ is the matrix having a lone 1 as its $(k, k)$-entry and all other entries 0 , and for each $k(1 \leq k \leq n)$, there exists a right ideal $I_{k}$ of $R$ such that $\left(e_{k k} L\right)_{M_{n}(R)}=I_{k}[k]$. If $I$ is a nonzero right ideal of $R$, then $I[1]$ is a nonzero right ideal of $M_{n}(R)$, so $L \cap I[1]=\left(I \cap I_{1}\right)[1] \neq 0$. This implies that $I \cap I_{1} \neq 0$. Hence $I_{1}$ is a f.g. essential right ideal of $R$. By Proposition 2.1.1, $I_{1}$ is a generator of Mod- $R$. We know $R$ is Morita equivalent to $M_{n}(R)$ via the Morita equivalence $G=\left(--\otimes_{M_{n}(R)} R^{n}\right)_{R}: \operatorname{Mod}-M_{n}(R) \longrightarrow$ Mod$R$. In particular, $G\left(\left(e_{11} L\right)_{M_{n}(R)}\right)=\left(e_{11} L \otimes_{M_{n}(R)} R^{n}\right)_{R}$. But we have a $R$-homomorphism $\phi:\left(e_{11} L \otimes_{M_{n}(R)} R^{n}\right)_{R} \rightarrow\left(I_{1}\right)_{R}$ which is defined by 


$$
\left(\begin{array}{ccc}
a_{1} & \ldots & a_{n} \\
0 & \ldots & 0 \\
\vdots & \ddots & \vdots \\
0 & \ldots & 0
\end{array}\right) \otimes\left(\begin{array}{c}
x_{1} \\
\vdots \\
x_{n}
\end{array}\right) \mapsto a_{1} x_{1}+\cdots+a_{n} x_{n} .
$$

Obviously $\phi$ is onto. Since $\left(I_{1}\right)_{R}$ is a generator of Mod- $R$, we infer that $\left(e_{11} L \otimes_{M_{n}(R)} R^{n}\right)_{R}$ is a generator of Mod- $R$. Hence $\left(e_{11} L\right)_{M_{n}(R)}$ is a generator of $\operatorname{Mod}-M_{n}(R)$ by [2, Prop.21.6]. Thus we have $L$ is a generator of Mod$M_{n}(R)$ because $e_{11} L$ is an image of $L$ as right $M_{n}(R)$-modules.

Lemma 2.1.4 Let $R$ be a right w-semi-Prüfer ring, $e$ an idempotent of $R$ with $R e R=R$. Then eRe is a right w-semi-Prüfer ring.

Proof. Clearly $e R e$ is a semiprime right Goldie ring. Suppose $L$ is a f.g. essential right ideal of $e R e$, we want to show that $L$ is a generator of Mod$e R e$. Write $L=\sum_{i}\left(e x_{i} e\right) e R e$. Then $L=L e R e=T e$, where $T=L e R$ is a f.g. right ideal of $R$. Clearly $T \subseteq e R$. We claim that $T_{R} \leq_{e}(e R)_{R}$. In fact, if $0 \neq e r \in e R$, then $e r R e \neq 0$ since $R$ is a semiprime ring. Hence $e r R e$ is a nonzero right ideal of $e R e$. Thus $\operatorname{er} \operatorname{Re} \cap L \neq 0$, i.e., $0 \neq$ erxe $\in L$ for some $x \in R$. So $0 \neq \operatorname{erxe} R \subseteq T$. Next we show that $T_{R}$ is a generator of Mod- $R$. We know $((1-e) R)_{R}$ has finite Goldie dimension, and so there exist nonzero uniform right ideals $U_{i}$ of $R$ such that

$$
U_{1}+\cdots+U_{n}=U_{1} \oplus \cdots \oplus U_{n} \leq_{e}((1-e) R)_{R}
$$

We claim $U_{i} e R \neq 0 \forall i$. Otherwise $e R \subseteq U_{i}^{\perp}=f R$ for some idempotent $f \in R$ by Proposition 2.1.2 and Theorem 1.6. Since $f R$ is an ideal, we have $R f \subseteq f R$ and so $(1-f) R f=0$. Since $R=R f+R(1-f)$, it follows 
that $R(1-f)$ is a two-sided ideal, and hence $f R(1-f)$ is a right ideal. Now $[f R(1-f)]^{2}=0$, and $R$ has no nonzero nilpotent right ideals, hence $f R(1-f)=0$. Given any $r \in R$, we thus have $f r(1-f)=0$ as well as $(1-f) r f=0$, whence $f r=f r f=r f$. Then $R=\operatorname{Re} R=R f R=R f$, and this implies that $f=1$. Therefore $U_{i}=U_{i} R=0$. The contradiction shows that $U_{i} e R \neq 0 \forall i$. Thus $e R U_{i} \neq 0 \forall i$ since $R$ is a semiprime ring. Since $T_{R} \leq_{e}(e R)_{R}$, we have $T \cap e R U_{i} \neq 0$. Then $0 \neq\left(T \cap e R U_{i}\right)^{2} \subseteq e R U_{i} T$. So $U_{i} T \neq 0 \forall i$. For each $i$, choose an $a_{i} \in U_{i}$ such that $a_{i} T \neq 0$. Then

$$
a_{1} T+\cdots+a_{n} T=a_{1} T \oplus \cdots \oplus a_{n} T \leq_{e}((1-e) R)_{R}
$$

Therefore

$$
T \oplus a_{1} T \oplus \cdots \oplus a_{n} T \leq_{e} e R \oplus(1-e) R=R_{R}
$$

By Proposition 2.1.1, $T \oplus a_{1} T \oplus \cdots \oplus a_{n} T$ is a generator of Mod- $R$. Since each $a_{i} T$ is an image of $T_{R}$, we conclude that $T$ is a generator of Mod$R$. To see $L$ is a generator of Mod-eRe, we use the Morita equivalence $\operatorname{Hom}_{R}(e R,--): \operatorname{Mod}-R \longrightarrow \operatorname{Mod}-e R e$. Since $T$ is a generator of Mod- $R$, we have $L_{e R e}=(T e)_{e R e} \cong\left(\operatorname{Hom}_{R}\left(e R, T_{R}\right)\right)_{e R e}($ by $[2$, Prop.4.6]) is a generator of Mod-eRe by [2, Prop. 21.6]. Finally, since $R$ is a semihereditary ring, every f.g. submodule of $(e R)_{R}$ is projective. Therefore $e R e=\operatorname{Hom}_{R}(e R, e R)$ is a right semihereditary ring by [2, Prop.21.6; Prop.21.8].

Theorem 2.1.1 The property of being a right w-semi-Prüfer (right semiPrüfer, right w-Prüfer, or right Prüfer) ring is a Morita invariant.

Proof. Suppose $R$ is a right $w$-semi-Prüfer ring which is Morita equivalent to $\operatorname{ring} S$. Then $S \cong e M_{n}(R) \epsilon$ for some $n$ and some idempotent $\epsilon \in M_{n}(R)$ 
with $M_{n}(R) e M_{n}(R)=M_{n}(R)$ by Theorem 1.5. By Lemma 2.1.3 and Lemma 2.1.4, $S$ is a right $w$-semi-Prüfer ring. Since the properties semiprime Goldie, prime right Goldie, and prime Goldie are all Morita invariants, the other parts follow immediately.

Proposition 2.1.3 The ring $R$ is a right semi-Prüfer (right w-semi-Prüfer, or right w-Prüfer, or right Prüfer) ring if and only if $R$ is a semiprime Goldie (semiprime right Goldie, or prime right Goldie, or prime Goldie) ring and every f.g. essential submodule of each progenerator of Mod- $R$ is a progenerator of Mod- $R$.

Proof. One direction is clear by Proposition 2.1.1. Suppose that $R$ is a right $w$-semi-Prüfer ring. Let $P_{R}$ be a progenerator and $N_{R}$ a f.g. essential submodule of $P_{R}$. And set $S=\operatorname{End}\left(P_{R}\right)$. Then we have the Morita equivalence $F=\operatorname{Hom}_{R}\left({ }_{S} P_{R},--\right): \operatorname{Mod}-R \longrightarrow \operatorname{Mod}-S$. By [2, Prop.21.6; Prop.21.8], $F(N)_{S}$ is a f.g. essential submodule of $F(P)_{S}=S_{S}$. We know $S$ is a right $w$-semi-Prüfer ring from Theorem 2.1.1. Hence it follows that $F(N)_{S}$ is a progenerator of Mod- $S$ from Proposition 2.1.1. Therefore $N_{R}$ is a progenerator of Mod- $R$ by [2, Prop.21.6; Prop.21.8].

Proposition 2.1.4 The following are equivalent for a ring $R$ :

(a) $R$ is a right $w$-Prüfer (or right Prüfer) ring;

(b) $R$ is a right Goldie (or Goldie) ring and every f.g. nonzero right ideal of $R$ is a progenerator of Mod-R;

(c) $R$ is a right Goldie (or Goldie) ring and every f.g. nonzero submodule of each progenerator of Mod-R is a progenerator of Mod-R. 
Proof. $(a) \Rightarrow(b)$. By Proposition 2.1.2, it is enough to show that every f.g. nonzero right ideal $I$ of $R$ is a generator. We can find a right ideal $J$ of $R$ such that $I+J=I \oplus J \leq_{e} R_{R}$. Since $R$ is a right Goldie ring, there exist uniform submodules $J_{1}, \ldots, J_{t}$ of $J_{R}$ such that $J_{1} \oplus \cdots \oplus J_{t} \leq_{e} J_{R}$. Hence $I \oplus J_{1} \oplus \cdots \oplus J_{t} \leq_{e} R_{R}$. Since $R$ is prime, $J_{i} I \neq 0$ for each $i$. So we can choose some $a_{i} \in J_{i}$ with $a_{i} I \neq 0$. Then $I \oplus a_{1} I \oplus \cdots \oplus a_{t} I \leq_{e} R_{R}$. By Proposition 2.1.1, $I \oplus a_{1} I \oplus \cdots \oplus a_{t} I$ is a generator of Mod- $R$. Therefore $I$ is a generator of Mod- $R$.

$(b) \Rightarrow(a)$. That every f.g. nonzero ideal of $R$ is a generator implies that $R$ is a prime ring.

$(b) \Rightarrow(c)$. Similar to the proof of Proposition 2.1.3.

$(c) \Rightarrow(a)$. By Proposition 2.1.1.

Proposition 2.1.5 The ring $R$ is a right w-semi-Prüfer ring if and only if $Z\left(R_{R}\right)=0, R_{R}$ is finite-dimensional and $R$ has Property $(\mathbf{P})$.

Proof. One direction is clear. Suppose that $Z\left(R_{R}\right)=0, R_{R}$ is finitedimensional, and $R$ has Property $(\mathbf{P})$. We only need to show that $R$ is semiprime right Goldie. Suppose $I^{2}=0$ for an ideal $I$ of $R$. We have a right ideal $J$ of $R$ such that $I+J=I \oplus J \leq_{e} R_{R}$. Then $(I+J) I \subseteq J I \subseteq I \cap J=0$. Since $R_{R}$ is finite-dimensional, there exist f.g. right ideals $I_{1}, J_{1}$ of $R$ such that $I_{1} \leq_{e} I_{R}, J_{1} \leq_{e} J_{R}$. Therefore we have $I_{1} \oplus J_{1} \leq_{e} R_{R}$. Since $R$ has Property (P), $I_{1} \oplus J_{1}$ is a generator of Mod- $R$. Thus $R_{R}$ is an $R$-homomorphic image of $\left(I_{1} \oplus J_{1}\right)^{(n)}$ for some $n$. Noting that $\left(I_{1} \oplus J_{1}\right) I=0$, we have $I=R I=0$. Therefore $R$ is semiprime. By Theorem 1.2, $R$ is a right Goldie ring. 
Proposition 2.1.6 The ring $R$ is a right w-Prüfer ring if and only if $R_{R}$ is finite-dimensional and every f.g. nonzero right ideal of $R$ is a progenerator of Mod-R.

Proof. The necessity follows from Proposition 2.1.4. For the converse, it is easy to see that $R$ is a prime ring. Suppose $Z\left(R_{R}\right) \neq 0$. We can choose a f.g. right ideal $I$ of $R$ such that $I \subseteq Z\left(R_{R}\right)$. By our assumption, $I$ is a generator of Mod- $R$, and thus $R_{R}$ is an epimorphic image of $I^{(n)}$ for some $n$ by Proposition 1.3. Since $I^{(n)}$ is singular, we have that $R_{R}$ is singular. This is a contradiction since $1 \notin Z\left(R_{R}\right)$. Therefore $Z\left(R_{R}\right)=0$, and thus $R$ is a prime right Goldie ring by Theorem 1.2.

Some other characterizations of right $w$-semi-Prüfer (right semi-Prüfer, right $w$-Prüfer, or right Prüfer) rings will be presented in the next chapter.

\subsection{A structure theorem and further properties of right $w$-semi-Prüfer rings}

Lemma 2.2.1 Let $R$ be a right w-semi-Prüfer ring, and $Q=Q_{c l}^{r}(R)$. If $e$ is a central idempotent of $Q$, then $(e R)_{R}$ is a projective $R$-module.

Proof. Write $e=u_{1} v^{-1}, 1-e=u_{2} v^{-1}$, where $u_{i} \in R$ and $v \in \mathcal{C}_{R}(0)$. Define a map $\phi: e R \oplus(1-e) R \longrightarrow R$ by $\phi(e x+(1-e) y)=u_{1} x+u_{2} y$ $\forall x, y \in R$. Suppose $e x+(1-e) y=e x^{\prime}+(1-e) y^{\prime}$. Then $e x=e x^{\prime}$, i.e., $\left(u_{1} v^{-1}\right) x=\left(u_{1} v^{-1}\right) x^{\prime}$. So $u_{1} x=v\left[\left(u_{1} v^{-1}\right) x\right]=v\left[\left(u_{1} v^{-1}\right) x^{\prime}\right]=u_{1} x^{\prime}$. Similarly $u_{2} y=u_{2} y^{\prime}$. Hence $u_{1} x+u_{2} y=u_{1} x^{\prime}+u_{2} y^{\prime}$. Thus $\phi$ is well defined. Clearly $\phi$ is a right $R$-module homomorphism. If $u_{1} x+u_{2} y=0$, then $0=$ 
$\left(u_{1} v^{-1}\right) v x+\left(u_{2} v^{-1}\right) v y=v\left[\left(u_{1} v^{-1}\right) x+\left(u_{2} v^{-1}\right) y\right]$, and then $e x+(1-e) y=0$. So $\phi$ is one to one. Therefore we have $(e R \oplus(1-e) R)_{R} \cong \operatorname{Im} \phi$. But $\operatorname{Im} \phi$ is a f.g. right ideal of $R$, and so it is projective by Proposition 2.1.2. Hence $(e R)_{R}$ is projective.

Proposition 2.2.1 Let $R, Q$ be as above, e any central idempotent of $Q$. Then $e \in R$.

Proof. Since $(1-e) R$ is a right projective $R$-module, the exact sequence $0 \rightarrow e R \cap R \rightarrow R \rightarrow(1-e) R \rightarrow 0$ splits. Then $e R \cap R$ is a direct summand of $R_{R}$. So we have $e R \cap R=f R$ for an idempotent $f \in R$. Then $f Q \subseteq e Q$. If $0 \neq e \xi \in e Q$, write $\xi=a c^{-1}$ for some $a \in R$ and $c \in \mathcal{C}_{R}(0)$. Then $0 \neq$ $(e \xi) c=e a \in e R$. Write $e a=u v^{-1}$ for some $u \in R$ and $v \in \mathcal{C}_{R}(0)$. We have $0 \neq(e \xi) c v=e a v=u \in e R \cap R=f R$, and so $0 \neq(e \xi) c v \in f Q$. Therefore $(f Q)_{Q} \leq_{e}(e Q)_{Q}$. Since $Q$ is a semi-simple Artinian ring, $(f Q)_{Q}$ is a direct summand of $(e Q)_{Q}$. It must be that $f Q=e Q$. Then $e=f e=e f=f$ since $e$ is central.

Proposition 2.2.2 Let $R$ be a right w-semi-Prüfer ring, $Q=Q_{c l}^{r}(R)=$ $Q_{1} \oplus \cdots \oplus Q_{n}$, where each $Q_{i}$ is a simple Artinian ring. Then $R=(R \cap$ $\left.Q_{1}\right) \oplus \cdots \oplus\left(R \cap Q_{n}\right)$, each $R \cap Q_{i}$ is a right $w$-Prüfer ring and $Q_{c l}^{r}\left(R \cap Q_{i}\right)=Q_{i}$.

Proof. By Theorem 1.1, $Q_{c l}^{r}(R)$ is a semi-simple Artinian ring. Hence the Wedderburn-Artin theorem asserts that $Q_{c l}^{r}(R)$ is a finite direct sum of simple Artinian rings: $Q_{c l}^{r}(R)=Q_{1} \oplus \cdots \oplus Q_{n}$, with each $Q_{i}$ being a simple Artinian ring. We have $1_{R}=1_{Q_{c l}^{r}(R)}=1_{Q_{1}}+\cdots+1_{Q_{n}}$, where $1_{Q_{i}}$ is the identity of $Q_{i}$. Set $R_{i}=R \cap Q_{i}$. Then $1_{Q_{i}} \in R_{i}$ by Proposition 2.2.1. Hence $R_{i}$ is a 
subring of $Q_{i}$. It is straightforward to check that each $R_{i}$ is a right order of $Q_{i}$. So $R_{i}$ is a prime right Goldie ring. Each $R_{i}$ is obviously an ideal of $R$, and for every $x \in R, x=x 1_{Q_{1}}+\cdots+x 1_{Q_{n}} \in R_{1} \oplus \cdots \oplus R_{n}$. Therefore we have $R=R_{1} \oplus \cdots \oplus R_{n}$. To see each $R_{i}$ is a right $w$-Prüfer ring, we only need to show that $R_{i}$ has property $(\mathbf{P})$ by Proposition 2.1.1. Let $I_{i}$ be a f.g. essential right ideal of $R_{i}$ and let $I=R_{1}+\cdots+R_{i-1}+I_{i}+R_{i+1}+\cdots+R_{n}=$ $R_{1} \oplus \cdots \oplus I_{i} \oplus \cdots \oplus R_{n}$. Then $I$ is a f.g. essential right ideal of $R$, and so $I_{R}$ is a progenerator of Mod- $R$ by Proposition 2.1.1. Hence $\left(I_{i}\right)_{R}$ is projective, and this implies that $\left(I_{i}\right)_{R_{i}}$ is projective. On the other hand, if $f \in \operatorname{Hom}\left(I_{R}, R_{R}\right)$, we have $f\left(R_{j}\right) \subseteq R_{j}$, if $j \neq i$, and $f\left(I_{i}\right) \subseteq R_{i}$. Since $I_{R}$ is a generator of $\operatorname{Mod}-R$,

$$
\begin{gathered}
R=\sum_{f \in \operatorname{Hom}\left(I_{R}, R_{R}\right)} I m f=\left(\sum_{f \in \operatorname{Hom}\left(I_{R}, R_{R}\right)} f\left(R_{1}\right)\right) \oplus \cdots \\
\cdots \oplus\left(\sum_{f \in \operatorname{Hom}\left(I_{R}, R_{R}\right)} f\left(I_{i}\right)\right) \oplus \cdots \oplus\left(\sum_{f \in \operatorname{Hom}\left(I_{R}, R_{R}\right)} f\left(R_{n}\right)\right) .
\end{gathered}
$$

So we have

$$
R_{i}=\sum_{f \in H o m\left(I_{R}, R_{R}\right)} f\left(I_{i}\right)=\sum_{f \in H o m\left(I_{i}, R_{i}\right)} f\left(I_{i}\right)=\sum_{f \in \operatorname{Hom}\left(I_{i R_{i}}, R_{i R_{i}}\right)} f\left(I_{i}\right) .
$$

It follows that $I_{i}$ is a generator of $\operatorname{Mod}-R_{i}$. Hence $I_{i}$ is a progenerator of Mod- $R_{i}$. We can conclude that each $R_{i}$ is a right $w$-Prüfer ring.

Theorem 2.2.1 $A$ ring $R$ is a right w-semi-Prüfer ring if and only if it is a finite direct sum of right w-Prüfer rings.

Proof. The necessity follows from Proposition 2.2.2.

Suppose $R=\bigoplus_{i=1}^{n} R_{i}$ be a direct sum of right $w$-Prüfer rings $R_{i}$. Then $Q_{c l}^{r}(R) \cong \bigoplus_{i=1}^{n} Q_{c l}^{r}\left(R_{i}\right)$ which is a semi-simple Artinian ring. Hence $R$ is a 
semiprime right Goldie ring. Suppose $I$ is a f.g. essential right ideal of $R$. Let $\pi_{i}$ be the $i^{t h}$ projection of $R$ onto $R_{i}$. We have $0 \neq I \cap R_{i} \subseteq \pi_{i}\left(I_{R}\right)$, and this implies that $\pi_{i}(I)$ is a f.g. essential right ideal of $R_{i}$. By Proposition 2.1.1, $\pi_{i}(I)$ generates $R_{i}$ as a right $R_{i}$-module, and thus $\pi_{i}(I)$ generates $R_{i}$ as a right $R$-module. Therefore we have shown that $I_{R}$ is a generator of Mod- $R$. Next instead of proving $I_{R}$ is projective, we show $R$ is a right semihereditary ring. For each $m$, let $M_{m}(R)=M_{m}\left(R_{1}\right) \oplus \cdots \oplus M_{m}\left(R_{n}\right)$. Given $x \in M_{m}(R)$, write $x=x_{1}+\cdots+x_{n}$, with each $x_{i} \in M_{m}\left(R_{i}\right)$. We want to show that $x M_{m}(R)$ is a projective right $M_{m}(R)$-module. Since each $M_{m}\left(R_{i}\right)$ is still a right $w$-Prüfer ring, we can assume $m=1$. Since $x_{i} R_{i}$ is a projective right $R_{i}$-module, we have $\left(R_{i}\right)_{R_{i}} \cong\left(x_{i} R_{i}\right)_{R_{i}} \oplus U_{i}$ for some right $R_{i}$-module $U_{i}$. We know $U_{i}$ can be regarded as a right $R$-module canonically. Thus as right $R$-modules we still have $\left(R_{i}\right)_{R} \cong\left(x_{i} R_{i}\right)_{R} \oplus U_{i}$. Therefore $\left(x_{i} R_{i}\right)_{R}$ is projective since $\left(R_{i}\right)_{R}$ is. Then $x R=x_{1} R+\cdots+x_{n} R=x_{1} R_{1} \oplus \cdots \oplus x_{n} R_{n}$ is a projective right $R$-module. We have actually shown that $x M_{m}(R)$ is a projective right $M_{m}(R)$-module for every $x \in M_{m}(R)$. By Proposition 1.5, $R$ is a right semihereditary ring.

Proposition 2.2.3 If $R$ is a right $w$-Prüfer ring, e a nonzero idempotent, then eRe is a right $w$-Prüfer ring.

Proof. Since $e R$ is a progenerator of Mod- $R$ by Proposition 2.1.4, then $e R e \cong$ $\operatorname{End}(e R)$ is Morita equivalent to $R$, thus is a right $w$-Prüfer ring by Theorem 2.1.1.

Corollary 2.2.1 If $R$ is a right w-semi-Prüfer ring, e a nonzero idempotent, then eRe is a right w-semi-Prüfer ring. 
Proof. By Theorem 2.2.1 and Proposition 2.2.3.

By a complete set of idempotents of a ring we mean a set of pairwise orthogonal idempotents: $\left\{e_{1}, \cdots, e_{t}\right\}$ with $\Sigma_{i=1}^{t} e_{i}=1$.

Proposition 2.2.4 If $R$ is a right w-Prüfer ring, then there exists a complete set of idempotents $e_{1}, \cdots, e_{n}$, such that $R=e_{1} R \oplus \cdots \oplus e_{n} R$ and for each $i, e_{i} R e_{i}$ is a right w-Prüfer domain which is Morita equivalent to $R$.

Proof. Since $R$ is a prime right Goldie ring, $R_{R}$ has the ascending chain condition (and the desending chain condition) on the set of direct summands of $R_{R}$ (see [2, Ex.§10.11]). By [2, Prop.10.14; Prop.7.2], there exists a complete set $e_{1}, \cdots, e_{m}$ of idempotents in $R$ such that $R=e_{1} R \oplus \cdots \oplus e_{m} R$ and each $e_{i} R$ is indecomposable as a right $R$-module. We know $e_{i} R$ is a progenerator by Proposition 2.1.4. Therefore $R$ is Morita equivalent to $e_{i} R e_{i} \cong$ $\operatorname{End}_{R}\left(e_{i} R\right)$. By Proposition 2.2.3, $e_{i} R e_{i}$ is also a right $w$-Prüfer ring. Now let $0 \neq x \in e_{i} R e_{i}$, then $x e_{i} R e_{i}$ is a projective $e_{i} R e_{i}$-module and it follows that $x^{\perp}=f e_{i} R e_{i}$ for some $f^{2}=f \in e_{i} R e_{i}$. But the ring $e_{i} R e_{i}$ has exactly one nonzero idempotent, namely $e_{i}$. It follows that $f=0$ or $e_{i}$. Since $x \neq 0$, it follows $f=0$, i.e., $x^{\perp}=0$ for all $0 \neq x \in e_{i} R e_{i}$. Therefore $e_{i} R e_{i}$ is a domain.

Lemma 2.2.2 Let $M_{1} \oplus M_{2} \oplus \cdots \oplus M_{n}=A \oplus B$ be a decomposition in $\operatorname{Mod}-R$ such that $\operatorname{End}\left(A_{R}\right)$ is a local ring. Then there exists $i, 1 \leq i \leq n$, and an isomorphism $M_{i} \cong A \oplus X$ for some $X \in \operatorname{Mod}-R$.

Proof. See [9, P39-40]. 
A module $M_{R}$ is called a quasi-injective module if for each submodule $N$ of $M$, every $R$-homomorphism from $N$ into $M$ can be extended to an $R$-homomorphism from $M$ into $M$.

Proposition 2.2.5 Let $R$ be a right w-Prüfer ring. If there exists a nonzero f.g. quasi-injective projective right $R$-module, then $R$ is a simple Artinian ring.

Proof. As in Proposition 2.2.4, $R=e_{1} R \oplus \cdots \oplus e_{n} R$, where each $e_{i} R$ is an indecomposable $R$-module. Let $M_{R}$ be a f.g. quasi-injective projective module. Since $e_{1} R$ is a generator, there exist an integer $m>0$ and some $R$-module $\mathrm{X}$ such that $\left(e_{1} R\right)^{m} \cong M \oplus X$. Since $R$ is finite-dimensional, $e_{1} R$, hence $\left(e_{1} R\right)^{m}$ has finite Goldie dimension. So $M$ has finite Goldie dimension. Write $M=M_{1} \oplus \cdots \oplus M_{k}$, where each $M_{i}$ is an indecomposable submodule of $M$. Now, if $M$ is a quasi-injective module, then each $M_{i}$ is a quasi-injective module. Therefore $\operatorname{End}\left(M_{i}\right)$ is a local ring. Thus Lemma 2.2 .2 implies that $e_{1} R \cong M_{1} \oplus U$, for some $U$. As we know $e_{1} R$ is an indecomposable $R$ module, we have $e_{1} R \cong M_{1}$ is quasi-injective. We can also show that each $e_{i} R \cong M_{1}$. Therefore $R_{R} \cong M_{1}^{n}$ is a quasi-injective module. Now Baer's Criterion implies that $R$ is a right self-injective ring. Then $R=E\left(R_{R}\right)$ is a semi-simple ring by [18, Th.4.28]. Hence $R$ is a simple Artinian ring.

We know that $\mathbf{Z}$, the ring of integers, is a Prüfer ring, but not a simple Artinian ring. We also know that $\mathbf{Q}$, the field of rational numbers, cannot be embedded in $\mathbf{Z}^{(I)}$ for any index set $I$. The following is one way to see this:

Corollary 2.2.2 If $R$ is a right w-Prüfer ring, but not simple Artinian, then, for any f.g. right $R$-module $M$, the injective hull $E(M)$ of $M$ cannot 
be embedded in a free $R$-module.

Proof. Suppose $M$ is a f.g. $R$-module, and $0 \longrightarrow E(M) \stackrel{l}{\longrightarrow} R^{(I)}$ is exact for some $I$. Since $M_{R}$ is finitely generated, $l(M) \subseteq R^{(F)}$, where $F$ is a finite subset of $I$. Let $p: R^{(I)} \longrightarrow R^{(F)}$ be the canonical projection. We consider $\operatorname{Ker}(p \circ l)$. Since $\operatorname{Ker}(p \circ l) \cap M=0$, and $M \leq_{e} E(M)$, we conclude that $\operatorname{Ker}(p \circ l)=0$. Thus $E(M)$ is embedded in $R^{(F)}$. But $E(M)$ is injective, so it is a direct summand of $R^{(F)}$, and therefore finitely generated. Now the previous proposition implies that $R$ is a simple Artinian ring.

\subsection{Prüfer rings and semi-Prüfer rings}

Proposition 2.3.1 Let $R$ be a right semi-Prüfer ring, and $Q_{c l}^{r}(R)=Q_{1} \oplus$ $\cdots \oplus Q_{n}$, where each $Q_{i}$ is a simple Artinian ring. Then $R=\left(R \cap Q_{1}\right) \oplus \cdots \oplus$ $\left(R \cap Q_{n}\right)$, where each $R \cap Q_{i}$ is a right Prüfer ring and $Q_{c l}^{r}\left(R \cap Q_{i}\right)=Q_{i}$.

Proof. Similar to the proof of Proposition 2.2.2.

Theorem 2.3.1 $A$ ring $R$ is a right semi-Prüfer ring if and only if $R$ is a finite direct sum of right Prüfer rings.

Proof. Similar to the proof of Theorem 2.2.1.

Next we turn to the left-right symmetry of Prüfer rings and semi-Prüfer rings.

Theorem 2.3.2 [1, Prop. 1.12]. A ring $R$ is a right Prüfer ring if and only if $R$ is a left Prüfer ring. 
Proof. Suppose $R$ is a right Prüfer ring. We want to show $R$ is left Prüfer. We know $R$ is a Goldie and left semihereditary ring by Proposition 2.1.2. So, to show $R$ is a left Prüfer ring, it suffices to show that for any f.g. nonzero left ideal $J$ of $R, J$ is a generator of $R$-Mod by using the left version of Proposition 2.1.4. Since $R$ is left semihereditary, $J$ is a projective left $R$-module. So we may assume that $R^{n}=J \oplus N$ for some $n$ and some $N \in R$-Mod. Therefore we have ${ }_{R} J=R^{n} f$ for some idempotent $f \in \operatorname{End}_{R}\left(R^{n}\right)=M_{n}(R)$. Since $R^{n}$ is a progenerator of $R$-Mod and $M_{n}(R)=\operatorname{End}_{R}\left(R^{n}\right)$, we have a Morita equivalence $R^{n} \otimes_{M_{n}(R)}--: M_{n}(R)$-Mod $\rightarrow R$-Mod. As left $R$-modules, $R^{n} \otimes_{M_{n}(R)} M_{n}(R) f \cong_{R}\left(R^{n} f\right)=_{R} J($ via $a \otimes b \rightarrow a b)$. So ${ }_{R} J$ is a generator of $R$-Mod if and only if $M_{n}(R)\left(M_{n}(R) f\right)$ is a generator of $M_{n}(R)$-Mod by [2, Prop.21.6]. Also, we know that $M_{n}(R)$ is a right Prüfer ring from Theorem 2.1.1. Therefore, without lose of generality, we may assume that $J=R e$ for some idempotent $e$ of $R$. Since $R$ is a prime ring, $R e R \leq_{e} R_{R}$. Then $\operatorname{Re} R \cap \mathcal{C}_{R}(0)$ is not empty by Proposition 1.2. Thus there exist elements $r_{i}, t_{i} \in R(i=1, \cdots, m)$ such that $x=r_{1} e t_{1}+\cdots+r_{m} e t_{m} \in \mathcal{C}_{R}(0)$. Consider right ideals $I=r_{1} e R+\cdots+r_{m} e R$ and $P=f R+x R$. Then $I$ is a f.g. fractional right ideal of $R$, and so $I^{-1} I=R$, since $R$ is right Prüfer. On the other hand, $P \subseteq R e R$, and $I=r_{1} e R+\cdots+r_{m} e R \subseteq r_{1} e R e R+\cdots+r_{m} e R e R=I e R \subseteq$ $T P$. From $P \subseteq R e R$ and $I \subseteq I P$, it follows that $R=I^{-1} I \subseteq I^{-1} I P \subseteq$ $I^{-1} I R e R=R R e R \subseteq R e R$. Hence $R=R e R$. Because the trace ideal $T\left({ }_{R} R e\right)$ is a two-sided ideal of $R$ and $T\left({ }_{R} R e\right)=\Sigma\left\{\phi(R e): \phi \in \operatorname{Hom}\left({ }_{R} R e,{ }_{R} R\right)\right\} \supseteq R e$, we have $R=R e R \subseteq T\left({ }_{R} R e\right) \subseteq R$. Therefore $R=T\left({ }_{R} R e\right)$. By (the left version of) Proposition $1.3, J=R e$ is a generator of $R$-Mod. 
Corollary 2.3.1 The ring $R$ is a right semi-Prüfer ring if and only if $R$ is left semi-Prüfer.

Proof. By Theorems 2.3.1, 2.3.2.

From now on we will use the terms Prüfer ring and semi-Prüfer ring instead of right (or left) Prüfer ring and right (or left) semi-Prüfer ring respectively.

Proposition 2.3.2 If $R$ is a Prüfer ring and e is a nonzero idempotent, then eRe is a Prüfer ring.

Proof. By Proposition 2.1.4, $e R$ is a progenerator of Mod- $R$, and so $e R e \cong$ $\operatorname{End}\left(e R_{R}\right)$ is Morita equivalent to $R$. It follows that $e R e$ is a Prüfer ring from Theorem 2.1.1.

Corollary 2.3.2 If $R$ is a semi-Prüfer ring and $e$ is a nonzero idempotent, then eRe is a semi-Prüfer ring.

Proof. We may assume that $R=R_{1} \oplus R_{2} \oplus \cdots \oplus R_{n}$ with each $R_{i}$ a Prüfer ring and $e=e_{1}+\cdots+e_{t}$ with $t \leq n$ and each $e_{i}$ a nonzero idempotent of $R_{i}$. Then $e R e=e_{1} R_{1} e_{1} \oplus \cdots \oplus e_{t} R_{t} e_{t}$. The previous proposition implies that each $e_{i} R_{i} e_{i}$ is a Prüfer ring. Hence Theorem 2.3.1 implies that $e R e$ is a semi-Prüfer ring.

Proposition 2.3.3 [1]. Each overring of a Prüfer ring is a Prüfer ring.

Proof. Let $R$ be a Prüfer ring, $Q=Q_{c l}(R)$, and let $S$ be an overring of $R$, i.e., $S$ is a subring of $Q$ such that $R \subseteq S \subseteq Q$. Clearly $S$ is right and left order in $Q$. Hence $S$ is a prime Goldie ring with $Q_{c l}(S)=Q$. Suppose that $J$ 
is a f.g. fractional right ideal of $S$, e.g., $J=a_{1} S+\cdots+a_{n} S$. We may assume that $a_{1}$ is a unit of $Q$. Consider $I=a_{1} R+\cdots+a_{n} R$. Then $I$ is a fractional right ideal of $R$, and thus $I^{-1} I=R$ and $I I^{-1}=O_{l}(I)$. Now we have $I S=J$ and $S=R S=\left(I^{-1} I\right) S=I^{-1} J$. The last equality implies that $T\left(J_{S}\right)=S$, i.e., $J$ is a generator of Mod-S. Therefore, by Lemma 2.1.2, $J^{-1} J=S$. On the other hand, $J I^{-1} J=J I^{-1} I S=J R S=J$. This implies that $I^{-1} \subseteq J^{-1}$. Hence $1 \in O_{l}(I)=I I^{-1} \subseteq J J^{-1}$. Then $O_{l}(J) \subseteq\left(O_{l}(J) J\right) J^{-1} \subseteq J J^{-1}$. Therefore $J J^{-1}=O_{l}(J)$. It follows that $S$ is a Prüfer ring.

Corollary 2.3.3 Each overring of a semi-Prüfer ring is a semi-Prüfer ring.

Proof. Let $R$ be a semi-Prüfer ring, and $Q_{c l}(R)=Q_{1} \oplus \cdots \oplus Q_{n}$, where each $Q_{i}$ is a simple Artinian ring. Then by Proposition 2.3.1, $R=\left(R \cap Q_{1}\right) \oplus \cdots \oplus$ $\left(R \cap Q_{n}\right)$, and each $R \cap Q_{i}$ is a Prüfer ring with $Q_{c l}^{r}\left(R \cap Q_{i}\right)=Q_{i}$. Now if $S$ is an overring of $R$, then $S \cap Q_{i}$ is an overring of $R \cap Q_{i}$. By Proposition 2.3.3, $S \cap Q_{i}$ is a Prüfer ring. But it is easy to see $S=\left(S \cap Q_{1}\right) \oplus \cdots \oplus\left(S \cap Q_{n}\right)$. It follows from Theorem 2.3.1 that $S$ is a semi-Prüfer ring.

The concept of a prime Dedekind ring was first introduced by Robson in [32] by the term "maximal order". An important characterization of the prime Dedekind rings of Robson is stated as follows: A ring $R$ is a prime Dedekind ring if and only if every nonzero submodule of a (left or right) progenerator is also a progenerator [29, Th.2.10, P.140]. It was proved in [1] that a ring is a Prüfer ring and a bounded Krull ring if and only if it is a prime Dedekind ring, where a bounded Krull ring is defined in the sense of Marubayashi (cf.[27] Sec.1).

Theorem 2.3.3 The following are equivalent for a ring $R$ : 
(a) $R$ is a Prüfer and (both sides) Noetherian ring;

(b) $R$ is a prime Dedekind ring.

Proof. $(b) \Rightarrow(a)$. Every one-sided ideal of $R$ is a progenerator, and hence a f.g. $R$-module. It follows that $R$ is a Noetherian ring. Thus Proposition 2.1.4 implies that $R$ is a Prüfer ring.

$(a) \Rightarrow(b)$. Let $P_{R}$ be a progenerator and $N_{R}$ nonzero submodule of $P_{R}$. We want to show that $N_{R}$ is also a progenerator. Let $S=\operatorname{End} P_{R}$. Then $R$ is Morita equivalent to $S$ via the Morita equivalence $F=\operatorname{Hom}_{R}\left({ }_{S} P_{R},--\right)$ : Mod- $R \longrightarrow \operatorname{Mod}-S$, and $F(N)_{S}$ is a nonzero right ideal of $S . S$ is also a Prüfer ring. Since the property of being a one-sided Noetherian ring is a Morita invariant, $S$ is a right Noetherian ring. So $F(N)_{S}$ is a nonzero f.g. right ideal of $S$. By Proposition 2.1.4, $F(N)_{S}$ is a progenerator of Mod-S. Hence $N_{S}$ is a progenerator of Mod- $R$. So $R$ is a prime Dedekind ring.

Corollary 2.3.4 $A$ ring is a semi-Prüfer Noetherian ring if and only if it is a finite direct sum of prime Dedekind rings.

Proof. This follows from Theorems 2.3.1, 2.3.3.

A ring $R$ is right bounded if every essential right ideal of $R$ contains an ideal which is essential as a right ideal. Note that a prime ring $R$ is right bounded if and only if every essential right ideal of $R$ contains a nonzero ideal. A right and left bounded ring is called a bounded ring.

Proposition 2.3.4 The ring $R$ is a right bounded semi-Prüfer ring if and only if $R$ is a finite direct sum of right bounded Prüfer rings. 
Proof. $(\Rightarrow)$. By Theorem 2.3.1, $R=R_{1} \oplus \cdots \oplus R_{n}$, with each $R_{i}$ being a Prüfer ring. Given an essential right ideal $I_{i}$ of $R_{i}$. Then $I=R_{1} \oplus \cdots \oplus$ $R_{i-1} \oplus I_{i} \oplus R_{i+1} \oplus \cdots \oplus R_{n}$ is an essential right ideal of $R$. Hence there exists an ideal $J$ of $R$ such that $J \subseteq I$ and $J_{R} \leq_{e} R_{R} . J \cap R_{i}$ is an ideal of $R_{i}$, and we have $0 \neq J \cap R_{i} \subseteq I \cap R_{i} \subseteq I_{i}$. Therefore $R_{i}$ is right bounded.

$(\Leftarrow)$. Let $R=R_{1} \oplus \cdots \oplus R_{n}$, where each $R_{i}$ is a right bounded Prüfer ring. Then $R$ is a semi-Prüfer ring by Theorem 2.3.1. Suppose $I_{R}$ is an essential right ideal of $R$. We need to show that $I_{R}$ contains an ideal of $R$ which is essential as a right ideal. It is easy to see that $I \cap R_{i} \leq_{e}\left(R_{i}\right)_{R_{i}}$. Hence for each $i$ there exists a nonzero ideal $K_{i}$ of $R_{i}$ such that $K_{i} \subseteq I \cap R_{i}$ and $\left(K_{i}\right)_{R_{i}} \leq_{e}\left(R_{i}\right)_{R_{i}}$. Hence $\left(K_{i}\right)_{R} \leq_{e}\left(R_{i}\right)_{R}$. Set $K=K_{1} \oplus \cdots \oplus K_{n}$. Then $K$ is an ideal of $R, K \subseteq I$ and $K_{R} \leq_{e} R_{R}$.

A module $M_{R}$ is faithful if for every $0 \neq r \in R, M r \neq 0$. Every generator is faithful. But the converse is not true. We call a ring a right $F P F$ ring if every f.g. right faithful module is a generator. An FPF ring is defined to be a left and right $F P F$ ring. There are some known relations between bounded prime Dedekind rings and prime FPF rings. In fact a bounded prime Dedekind ring can be characterized as a Noetherian prime right (or left) FPF ring [10, Th.4.6]. In the following we point out how a bounded Prüfer (or semi-Prüfer) ring is related to an $F P F$ ring.

Theorem 2.3.4 For a ring $R$, the following are equivalent:

(a) $R$ is a prime right FPF right semihereditary ring;

(b) $R$ is a right bounded Prüfer ring;

(c) $R$ is a prime right FPF left semihereditary ring. 
Proof. (b) $\Rightarrow(a) \&(c)$. If $R$ is a right bounded Prüfer ring, then $R$ is prime Goldie semihereditary ring. Moreover, every f.g. nonzero right ideal is a generator by Proposition 2.1.4. Now, by [10, Th.4.7], $R$ is a prime right $F P F$ ring. So $(b)$ implies $(a)$ and $(c)$.

$(a) \Rightarrow(b)$. If $R$ is a prime $F P F$ right semihereditary ring, then, by [10, Th.4.7], $R$ is a right bounded Goldie (both sides) ring and every nonzero f.g. right ideal is a generator. Now, since $R$ is also a right semihereditary ring, it follows that every nonzero f.g. right ideal of $R$ is a progenerator. By Proposition 2.1.4, $R$ is a Prüfer ring.

$(a) \Leftarrow(c)$. From the proof above, we know any ring $R$ which possesses (c) must be a right bounded prime Goldie ring for which every f.g. nonzero right ideal is a generator. Since the property of being a prime Goldie ring is Morita invariant, $M_{n}(R)$ is prime Goldie ring for all $n$. In particular, $M_{n}(R)$ does not possess an infinite set of orthogonal idempotents. So Theorem 1.7 implies that $R$ is right semihereditary ring, and so $(a)$ holds.

Corollary 2.3.5 For a ring $R$, the following are equivalent:

(a) $R$ is a prime FPF left semihereditary ring;

(b) $R$ is a bounded Prüfer ring;

(c) $R$ is a prime FPF right semihereditary ring.

Corollary 2.3.6 Every right bounded semi-Prüfer ring $R$ is a semiprime semihereditary right FPF ring; The converse is true if $R$ also has ACC on annihilators.

Proof. For the first part, it is enough to show that $R$ is a right $F P F$ ring. By Proposition 2.3.4, $R=R_{1} \oplus \cdots \oplus R_{n}$, with each $R_{i}$ being a right bounded 
Prüfer ring. Then each $R_{i}$ is a right $F P F$ ring by Theorem 2.3.4. Therefore we have $R$ is a right $F P F$ ring by [10, Th.3.4]. For the second part, we first note that $R$ is a Goldie ring by [10, Cor.3.16C]. Then [10, Th.3.4(1)] implies that $R=R_{1} \oplus \cdots \oplus R_{n}$, where each $R_{i}$ is a prime right $F P F$ ring. Since $R$ is a semihereditary ring, it is easy to show that each $R_{i}$ is also a semihereditary ring. By Theorem 2.3.4, $R_{i}$ is a right bounded Prüfer ring. Now Proposition 2.3.4 implies that $R$ is a right bounded semi-Prüfer ring.

It was proved that every Prüfer ring is Morita equivalent to a Prüfer domain in $[1$, Th.2.3]. We give the following stronger result:

Theorem 2.3.5 Let $R$ be a Prüfer ring. Then there exists a complete set of idempotents $e_{1}, \cdots, e_{n}$ such that $R=e_{1} R \oplus e_{2} R \oplus \cdots \oplus e_{n} R$, where for each $i, e_{i} R$ is a uniform R-module, $e_{i} R e_{i}$ is a Prüfer domain and $R$ is Morita equivalent to $e_{i} R e_{i}$.

Proof. By Proposition 2.2.4, it is enough to show that $e R$ is a uniform right $R$-module for each indecomposable module $e R$. Let $N$ be a nonzero $R$-submodule of $e R$. We want to show that $N$ is an essential submodule of $e R$. Suppose $N \cap K=0$ for a submodule $K$ of $e R$. We know that there exists a submodule $L$ of $e R$ which is maximal with respect to $K \subseteq L$ and $N \cap L=0$. By Proposition 1.1, $N$ is embedded in $e R / L$ as an essential submodule. From Theorem 1.2, we have $Z\left(R_{R}\right)=0$, and thus $Z\left(N_{R}\right)=0$. It follows that $Z(e R / L)=0$. By noting Proposition 1.2, we have that $e R / L$ is a f.g. torsionfree right $R$-module. Therefore, it follows from Theorem 1.3 that $e R / L$ is embedded in a f.g. free right $R$-module. Since $R$ is Prüfer, $e R / L$ is a projective right $R$-module. Thus $e R \cong L \oplus(e R / L)$. Now the 
indecomposablity of $e R$ implies that $L=0$. Hence $K=0$, and $N$ is essential in $e R$.

Lemma 2.3.1 Let $R=R_{1} \oplus \cdots \oplus R_{n}$ and $S=S_{1} \oplus \cdots \oplus S_{n}$. If $R_{i}$ is Morita equivalent to $S_{i}(i=1, \cdots, n)$, then $R$ is Morita equivalent to $S$.

Proof. Well-known.

Corollary 2.3.7 Every semi-Prüfer ring is Morita equivalent to a finite direct sum of Prüfer domains.

Proof. By Theorem 2.3.1, Lemma 2.3.1 and Theorem 2.3.5.

In the final part of this section, we consider semiperfect Prüfer rings. A ring $R$ is semiperfect if $R / \operatorname{Rad}(R)$ is semi-simple, and idempotents of $R / \operatorname{Rad}(R)$ lift. By a theorem of Bass, the ring $R$ is semiperfect if and only if there exists a complete set of primitive idempotents $e_{1}, \cdots, e_{n}$ such that $R=e_{1} R \oplus \cdots \oplus e_{n} R$ and each $e_{i} R e_{i}$ is local, where a primitive idempotent is any idempotent which cannot be written as the sum of two nontrivial orthogonal idempotents (see [2,Th.27.6]). For any semiperfect ring $R$, there exists a basic set of orthogonal primitive idempotents $\left\{e_{1}, \cdots, e_{t}\right\}$ in the sense that for every primitive idempotent $f$ we have $R f \cong R e_{i}$ for exactly one $e_{i}$, $1 \leq i \leq t$. In this case $e=\sum_{i=1}^{t} e_{i}$ is called a basic idempotent and $e R e$ is called the basic ring of $R$. A module is uniserial if its submodules are linearly ordered with respect to inclusion. A ring $R$ is right serial if $R_{R}$ is a direct sum of uniserial modules. The ring is serial if it is both left and right serial. A local serial ring is called a valuation ring. 
Lemma 2.3.2 Let $R$ be a Prüfer ring, $e^{2}=e \in R$. If eRe is a local ring, then $R$ is a semiperfect ring, and every indecomposable projective right $R$ module is isomorphic to $e R, e R e$ is the basic ring of $R$, and $R \cong M_{n}(e R e)$, where $n$ is the Goldie dimension of $R_{R}\left(\right.$ or ${ }_{R} R$ ).

Proof. By Theorem 2.3.5, $R=e_{1} R \oplus \cdots \oplus e_{n} R$, where $n$ is the Goldie dimension of $R_{R}$, and $e_{i} R$ is indecomposable for all $i$. For each $i, e_{i} R$ is a generator and $e R$ is projective module, so we have $\left(e_{i} R\right)^{m} \cong e R \oplus X$ for some $m>0$ and some $R$-module $X$. Because $e R e$ is a local ring, Lemma 2.2.2 implies $e_{i} R \cong e R \oplus Y$ for some $Y$. Hence $e_{i} R \cong e R$ since $e_{i} R$ is indecomposable. And so $e_{i} R e_{i} \cong \operatorname{End}\left(e_{i} R\right) \cong \operatorname{End}(e R) \cong e R e$ is local ring. Hence $R$ is a semiperfect ring, and $R_{R} \cong(e R)^{n}$. So $R \cong \operatorname{End}\left((e R)^{n}\right) \cong$ $M_{n}(e R e)$. The other assertions follow from [2, Prop.27.10].

Lemma 2.3.3 Let $R$ be a Prüfer ring, $e^{2}=e \in R$. The following are equivalent:

(a) $(e R)_{R}$ is a uniserial module;

(b) eRe is a local ring;

(c) ${ }_{R}(R e)$ is a uniserial module.

Proof. $(a) \Rightarrow(b)$. Let $J=\operatorname{Rad}(R)$. We know $\operatorname{Rad}(e R)=e J$ is the intersection of all the maximal submodules of $e R$. Hence $e J$ is the unique maximal submodule of $e R$. By [2, Cor.17.20], $e R e$ is a local ring.

$(a) \Leftarrow(b)$. If $e R e$ is a local ring, then $R$ is a semiperfect ring by Lemma 2.3.2. By noting a result of Warfield which says a semiperfect semiprime Goldie ring is left semihereditary if and only if it is right serial [38, Cor.4.7], 
we have that $R$ is a right serial ring. Lemma 2.3.2 implies that every uniserial summand of $R_{R}$ is isomorphic to $e R$. So $e R$ is a uniserial module.

$(b) \Leftrightarrow(c)$. Similarly.

Corollary 2.3.8 Let $R$ be a Prüfer ring, then $R$ is a left (or right) serial ring if and only if $R$ is a semiperfect ring.

Proof. By Lemma 2.3.3.

Theorem 2.3.6 Let $R$ be a Prüfer ring.

(a) If $R$ is a local ring, then $R$ is a valuation Prüfer domain with both ${ }_{R} R$ and $R_{R}$ uniserial modules.

(b) If $R$ is a semiperfect ring, then $R$ is a serial ring and $R \cong M_{n}(B)$, where $n$ is the Goldie dimension of $R$ and $B$, its basic ring, is a valuation Prüfer domain.

Proof. (a). By Lemma 2.3.3.

(b). This follows from Lemmas 2.3.2, 2.3.3 and Corollary 2.3.8.

Corollary 2.3.9 The ring $R$ is a semiperfect semi-Prüfer ring if and only if it is a finite direct sum of matrix rings over valuation Prüfer domains.

Proof. $(\Rightarrow)$. By Theorem 2.3.1, $R=R_{1} \oplus \cdots \oplus R_{n}$, each $R_{i}$ is a Prüfer ring. If $R$ is a semiperfect ring, then every $R_{i}$ is semiperfect by [2, Coro.27.9]. Therefore we have $R_{i} \cong M_{n_{i}}\left(B_{i}\right)$ for some valuation Prüfer domain $B_{i}$ by Theorem 2.3.6.

$(\Leftarrow)$. A finite direct sum of matrix rings over valuation Prüfer domains is clearly a semiperfect ring, and is also a semi-Prüfer ring by Theorem 2.3.1. 
Finally, we give a characterization of f.g. torsionfree modules over a semi-Prüfer ring. A module $M_{R}$ is flat if whenever $f:{ }_{R} N_{1} \longrightarrow{ }_{R} N_{2}$ is a monomorphism, we have $1 \otimes f: M \otimes_{R} N_{1} \longrightarrow M \otimes_{R} N_{2}$ is a monomorphism.

Proposition 2.3.5 The following are equivalent for a module $M_{R}$ over a semi-Prüfer ring $R$ :

(a) $M$ is f.g. torsionfree;

(b) $M$ is f.g. flat;

(c) $M$ is f.g. projective;

(d) $M$ is projective with finite Goldie dimension;

(e) $M$ is a finite direct sum of f.g. uniform right ideals of $R$.

Proof. $(a) \Rightarrow(e)$. By Theorem 1.3, $M$ is a submodule of a f.g. free module $F_{R}$. Because of Theorem 2.3.1, we may assume $R=R_{1} \oplus \cdots \oplus R_{n}$, where each $R_{i}$ is a Prüfer ring. By Theorem 2.3.5, each $R_{i}$ is a finite direct sum of f.g. uniform right ideals of $R_{i}$. Since every f.g. uniform right ideal of $R_{i}$ is clearly a f.g. uniform right ideal of $R$, we have $F=\sum_{i=1}^{m} \oplus I_{i}$, where each $I_{i}$ is a f.g. uniform right ideal of $R$. Since $R$ is a semihereditary ring, every f.g. $R$-submodule of $I_{i}$ is projective. By [24, Prop.8, P85], $M \cong \sum_{i=1}^{m} \oplus N_{i}$, with each $N_{i} \subseteq I_{i}$.

$(d) \Leftarrow(e)$. This is because $R$ is a semihereditary Goldie ring.

$(c) \Leftarrow(d)$. Sandomierski showed in [32, Th.2.1] that if $R$ is a ring such that $Z\left(R_{R}\right)=0$ and $P_{R}$ is a projective module containing a f.g. essential submodule, then $P$ is finitely generated [33, Th.2.1]. Our claim follows.

$(b) \Leftarrow(c)$. Well-known. 
$(a) \Leftarrow(b)$. Suppose $M_{R}$ is flat and let $s \in \mathcal{C}_{R}(0)$. Define $\sigma_{s}: R \longrightarrow R$ by $\sigma_{s}(a)=a s$, which is a monomorphism as left $R$-modules. This gives rise to a commutative diagram

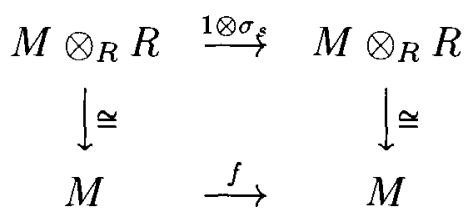

where $f(x)=x s$. Since $1 \otimes \sigma_{s}$ is a monomorphism, so is $f$, and thus $x \neq 0$ implies $x s \neq 0$. Therefore $M$ is torsionfree. 


\section{Strongly compressible modules}

Semiprime right Goldie rings constitute a much studied and well known family of rings, and satisfy one of basic conditions satisfied by right $w$-semi-Prüfer (right $w$-Prüfer, semi-Prüfer, or Prüfer) rings which were defined in chapter 2. Recently López-Permouth, Rizvi and Yousif [26] provided some interesting characterizations of semiprime Goldie rings in terms of their right ideals and of their nonsingular right modules. It was shown that a ring $R$ is semiprime Goldie if and only if every right ideal of $R$ is weakly-injective if and only if $R$ is right nonsingular and every nonsingular right $R$-module is weakly-injective [26, Th.3.9]. This motivates us to look for module-theoretic characterizations of semiprime right Goldie rings. Once such characterizations are established, it can be expected that one can present some new characterizations of right $w$-semi-Prüfer (right $w$-Prüfer, semi-Prüfer, or Prüfer) rings. In this chapter, we give the definition of strongly compressible modules. It turns out that the concept of strongly compressible modules is closely related to that of weaklyinjective modules and is precisely what we want for our purposes. In fact the connection between strongly compressible modules and weakly-injective modules is similar to that between compressible modules and tight modules (Proposition 3.2.1). We show that a ring $R$ is semiprime right Goldie if and only if $R_{R}$ is strongly compressible if and only if every right ideal of $R$ is strongly compressible if and only if every submodule of each progenerator of Mod- $R$ is strongly compressible (Theorem 3.1.1). As a corollary of this result, it is shown that a ring $R$ is semiprime Goldie if and only if every f.g. submodule of the injective hull of $R_{R}$ is strongly compressible if and only if $R$ is right 
nonsingular and every f.g. nonsingular right $R$-module is strongly compressible. This characterization theorem can easily imply the above-mentioned characterization theorem of López-Permouth, Rizvi and Yousif because of the strong connection between strongly compressible modules and weaklyinjective modules. In the latter part of the chapter, we apply our results to obtain some new module-theoretic characterizations of prime Goldie (prime right Goldie) rings, and right $w$-semi-Prüfer (right $w$-Prüfer, semi-Prüfer, or Prüfer) rings, respectively.

\subsection{New characterizations of semiprime right Goldie rings}

Following Jain and López-Permouth [20], a module $M$ is weakly-injective if and only if for every f.g. submodule $N$ of $E(M)$ there exists $X \subseteq E(M)$ such that $N \subseteq X \cong M$. In [23] a module $M$ is said to be compressible if it is embeddable in each of its essential submodules.

Definition 3.1.1 $A$ module $M_{R}$ is said to be strongly compressible if for every essential submodule $N$ of $M$ there exists $X \subseteq E(M)$ such that $M \subseteq$ $X \cong N$.

Every essential submodule of a strongly compressible module is strongly compressible. Every strongly compressible module is clearly compressible. After Theorem 3.1.5, we will give an example of a compressible module which is not strongly compressible. 
Lemma 3.1.1 Every f.g. strongly compressible right module has finite Goldie dimension.

Proof. Let $M_{R}$ be a f.g. strongly compressible module. Suppose $M_{R}$ is not finite-dimensional. Then there exists an essential submodule $N$ of $M$ such that $N=\oplus_{i=1}^{\infty} N_{i}$, where each $N_{i} \neq 0$. Since $M_{R}$ is strongly compressible, there exists a submodule $X$ of $E\left(M_{R}\right)$ such that $M \subseteq X \cong N$. Then $X=\bigoplus_{i=1}^{\infty} X_{i}$ and $\left(X_{i}\right)_{R} \cong\left(N_{i}\right)_{R}$ for all $i$. Clearly $M \subseteq \bigoplus_{i=1}^{k} X_{i}$ for some $k$. Thus $M \cap X_{i}=0$ for all $i>k$, contradicting the essentiality of $M$ in $E\left(M_{R}\right)$.

Lemma 3.1.2 Let $P_{R}$ be a progenerator of $M o d-R$. If $P_{R}$ is strongly compressible, then $R$ is semiprime.

Proof. Since $P_{R}$ is a progenerator of Mod- $R$, we can assume that $P^{n}=R \oplus X$ and $R^{m}=P \oplus Y$ for some positive integers $n, m$ and some $X, Y \in \operatorname{Mod}-$ $R$. If $I^{2}=0$ for some ideal $I$ of $R$, then $I \subseteq{ }^{\perp} I$. There exists a right ideal $J$ of $R$ maximal with respect to ${ }^{\perp} I \cap J=0$. Then ${ }^{\perp} I \oplus J \leq_{e} R_{R}$. $J I \subseteq J \cap I \subseteq J \cap^{\perp} I=0$. Then $J \subseteq J \cap^{\perp} I=0$. Hence ${ }^{\perp} I \leq_{e} R_{R}$. Therefore we have $\left({ }^{\perp} I\right)^{m} \leq_{e} R^{m}$, and thus $\left({ }^{\perp} I\right)^{m} \cap P \leq_{e} P$. Since $P_{R}$ is strongly compressible, there exists $Z_{R} \subseteq E\left(P_{R}\right)$ such that $P \subseteq Z \cong\left({ }^{\perp} I\right)^{m} \cap P$. Then $P I \subseteq Z I \cong\left(\left({ }^{\perp} I\right)^{m} \cap P\right) I=0$. So $P I=0$. Then $P^{n} I=0$. It implies that $R I=0$. Therefore $I=0$.

Lemma 3.1.3 Let $P_{R}$ be a progenerator of Mod-R. If $P_{R}$ is strongly compressible, then $Z\left(P_{R}\right)=0$. In particular, $Z\left(R_{R}\right)=0$.

Proof. We can assume that $R^{m}=P \oplus X$ for some positive integer $m$ and some $X \in \operatorname{Mod}-R$. There exists a submodule $N$ of $P$ such that $Z\left(P_{R}\right) \oplus N \leq_{e} P$. 
Since $P$ is strongly compressible, there exists $Y_{R} \subseteq E\left(P_{R}\right)$ such that $P \subseteq$ $Y \cong Z\left(P_{R}\right) \oplus N$. Write $Y=Y_{1} \oplus Y_{2}$ with $Y_{1} \cong Z\left(P_{R}\right)$ and $Y_{2} \cong N$ as right $R$-modules. For each $i(1 \leq i \leq m)$, let $e_{i}$ be the element of $R^{m}$ with $i^{\text {th }}$ component 1 and all others 0 . Write $e_{i}=a_{i}+b_{i}$ for some $a_{i} \in Y_{1}$ and $b_{i} \in Y_{2} \oplus X$. Since $Y_{1}$ is right singular, $a_{i}^{\frac{1}{1}} \leq_{e} R_{R}$. And $e_{i} a_{i}^{\perp}=b_{i} a_{i}^{\perp} \subseteq Y_{2} \oplus X$ for $i=1,2, \cdots, m$. It is easy to see that $\left(e_{i} a_{i}^{\perp}\right)_{R} \cong\left(a_{i}^{\perp}\right)_{R}$ and $\sum_{1}^{m} e_{i} a_{i}^{\perp}$ is a direct sum. Noting that $P_{R}$, and hence $R_{R}$ has finite Goldie dimension by Lemma 3.1.1, we have that $\operatorname{dim}\left(R^{m}\right)=\operatorname{dim}(P \oplus X)=\operatorname{dim}(P)+\operatorname{dim}(X)=$ $\operatorname{dim}(Y)+\operatorname{dim}(X)=\operatorname{dim}\left(Y_{1}\right)+\operatorname{dim}\left(Y_{2}\right)+\operatorname{dim}(X)=\operatorname{dim}\left(Y_{1}\right)+\operatorname{dim}\left(Y_{2} \oplus\right.$ $X) \geq \operatorname{dim}\left(Y_{1}\right)+\operatorname{dim}\left(\sum_{1}^{m} \oplus e_{i} a_{i}^{\perp}\right)=\operatorname{dim}\left(Y_{1}\right)+\sum \operatorname{dim}\left(e_{i} a_{i}^{\perp}\right)=\operatorname{dim}\left(Y_{1}\right)+$ $\sum \operatorname{dim}\left(a_{i}^{\perp}\right)=\operatorname{dim}\left(Y_{1}\right)+m \cdot \operatorname{dim}(R)=\operatorname{dim}\left(Y_{1}\right)+\operatorname{dim}\left(R^{m}\right)$. Thus $\operatorname{dim}\left(Y_{1}\right)=0$, i.e., $Y_{1}=0$. Therefore $Z\left(P_{R}\right)=0$.

Lemma 3.1.4 [Jategaonkar]. Let $R$ be a semiprime right Goldie ring. Then any submodule of a f.g. free right $R$-module is compressible.

Proof. Since $R_{R}$ is finite-dimensional, there exist f.g.uniform right ideals of $R$ whose sum, say $K$, is direct and essential in $R$. By Proposition $1.2, K$ contains a regular element $r$ of $R$. Clearly, the map $a \mapsto r a, a \in R$, embeds $R_{R}$ in $K$. It follows that any f.g. free right $R$-module can be embedded in a finite direct sum of f.g. uniform right ideals of $R$. Then, if $M$ is a submodule of a f.g. free right $R$-module, there exist f.g. uniform right ideals of $R: I_{1}, \cdots, I_{n}$ such that $M \subseteq \oplus_{i=1}^{n} I_{i}$. If $M \cap I_{j}=0$ for some $j$, then the restriction of the obvious map $\oplus_{i=1}^{n} I_{i} \longrightarrow \oplus_{i \neq j} I_{i}$ embeds $M$ in $\oplus_{i \neq j} I_{i}$. Thus, after omitting some of the modules $I_{i}$ and then reindexing, we may assume that $M \cap I_{i} \neq 0$ for all $i$. It follows that $M$ is essential in $\oplus_{i=1}^{n} I_{i}$. Let $N$ be an 
essential submodule of $M$. Then $N \cap I_{i} \neq 0$ for all $i$. Since $R$ is semiprime, we have $\left(N \cap I_{i}\right) I_{i} \neq 0$. Thus, $t I_{i} \neq 0$ for some $t \in N \cap I_{i}$. Now, consider the $R$-homomorphism $f: I_{i} \longrightarrow N \cap I_{i}$ defined by $f(b)=t b$. If $K \operatorname{er}(f) \neq 0$, then $\operatorname{Ker}(f) \leq_{e} I_{i}$, and so $N \cap I_{i} \cong I_{i} / \operatorname{Ker}(f)$ is torsion by Proposition 1.2. This is impossible because $N \cap I_{i}$ is torsionfree. So $f$ is a monomorphism. Clearly, the map $\oplus f_{i}: \oplus I_{i} \longrightarrow \oplus\left(N \cap I_{i}\right)$ provides an embedding of $M$ into $N$.

Now we can characterize semiprime right Goldie rings as follows.

Theorem 3.1.1 The following are equivalent for a ring $R$ :

(a) $R$ is semiprime right Goldie;

(b) $R_{R}$ is strongly compressible;

(c) Every cyclic right ideal of $R$ is strongly compressible;

(c') Every cyclic essential right ideal of $R$ is strongly compressible;

(d) Every f.g. right ideal of $R$ is strongly compressible;

(d') Every f.g. essential right ideal of $R$ is strongly compressible;

(e) Every right ideal of $R$ is strongly compressible;

(e') Every essential right ideal of $R$ is strongly compressible;

(f) Every cyclic submodule of each progenerator of Mod-R is strongly compressible;

(f') Every cyclic essential submodule of each progenerator of Mod-R is strongly compressible;

(g) Every f.g. submodule of each progenerator of Mod- $R$ is strongly compressible; 
(g') Every f.g. essential submodule of each progenerator of Mod-R is strongly compressible;

(h) Every submodule of each progenerator of Mod- $R$ is strongly compressible;

(h') Every essential submodule of each progenerator of Mod- $R$ is strongly compressible.

Proof. $(e) \Rightarrow(d) \Rightarrow(c) \Rightarrow(b)$ and $(e) \Rightarrow\left(e^{\prime}\right) \Rightarrow\left(d^{\prime}\right) \Rightarrow\left(c^{\prime}\right) \Rightarrow(b)$. Obviously.

$(b) \Rightarrow(a)$. By Lemmas 3.1.1, 3.1.2 and 3.1.3.

$(a) \Rightarrow(e)$. Let $I$ be a right ideal of $R$ and $K_{R} \leq_{e} I_{R}$. There exists $J \subseteq R_{R}$ such that $I \cap J=0$ and $I \oplus J \leq_{e} R_{R}$. Then $K \oplus J \leq_{e} R_{R}$. By Proposition $1.2, K \oplus J$ contains a regular element $r$ of $R$. Then the map $f:(r I)_{R} \rightarrow I_{R}$, which is defined by $f(r x)=x$ for all $x \in I$, is an isomorphism. Since $K_{R} \leq_{e} I_{R}$, we have $(r K)_{R} \leq_{e}(r I)_{R}$ by Proposition 1.1. Since $R$ is semiprime right Goldie, as a submodule of $R_{R},(r I)_{R}$ is compressible by Lemma 3.1.4. Hence there exists a monomorphism $g:(r I)_{R} \rightarrow(r K)_{R}$. Since $E(I)$ is an injective module, there exists $h:(r K)_{R} \rightarrow E(I)$, such that $h \circ g=f$. Since $(r K)_{R} \leq_{e}(r I)_{R}$, we have $\operatorname{dim}(r K)_{R}=\operatorname{dim}(r I)_{R}=\operatorname{dim}(g(r I))$, and thus $g(r I) \leq_{e}(r K)_{R}$. Then $h$ is one to one since $f$ is an isomorphism and $g$ is one to one. Let $X=h(r K)$. Then $I=f(r I)=h \circ g(r I) \subseteq h(r K)=X \subseteq E(I)$, and $X_{R} \cong(r K)_{R} \cong K_{R}$. Therefore $I_{R}$ is strongly compressible.

$(h) \Rightarrow(g) \Rightarrow(f) \Rightarrow(b)$ and $(h) \Rightarrow\left(h^{\prime}\right) \Rightarrow\left(g^{\prime}\right) \Rightarrow\left(f^{\prime}\right) \Rightarrow(b)$. Obviously.

$(a) \Rightarrow(h)$. Suppose that $P_{R}$ is a progenerator of $\operatorname{Mod}-R, N$ a submodule of $P$ and $K_{R} \leq_{e} N_{R}$. Set $S=\operatorname{End}\left(P_{R}\right)$. Then we have the Morita 
equivalence $F=\operatorname{Hom}_{R}\left({ }_{S} P_{R},--\right): \operatorname{Mod}-R \longrightarrow \operatorname{Mod}-S$ with inverse equivalence $G=\left(--\otimes_{S} P\right)_{R}: \operatorname{Mod}-S \leftarrow \operatorname{Mod}-R$. By [2, Prop.21.6], we have $F(K)_{S} \leq_{e} F(N)_{S} \subseteq F(P)_{S}=S_{S}$. We know that the property of being a semiprime right Goldie ring is Morita invariant, and thus $S$ is a semiprime right Goldie ring. By the equivalence of $(a)$ and $(e)$, we have $F(N)$ is a right strongly compressible $S$-module. Hence there exists $Y_{S} \subseteq E\left(F(N)_{S}\right)$ such that $F(N)_{S} \subseteq Y_{S} \cong F(K)_{S}$. Then $G F(N) \subseteq G(Y) \cong G F(K) \cong K_{R}$ and $G(Y) \subseteq G(E(F(N)))$. Noting that $F(N) \subseteq F(E(N))$ and $F(E(N))$ is injective [2, Prop.21.6], we have $E(F(N)) \subseteq F(E(N))$. Hence $E(F(N))=$ $F(E(N))$, since $\operatorname{dim} E(F(N))_{S}=\operatorname{dim} F(N)_{S}=\operatorname{dim} N_{R}=\operatorname{dim} E(N)_{R}=$ $\operatorname{dim} F(E(N))_{S}<\infty$ by [2, Prop.21.7]. So $G(E(F(N)))=G F(E(N)$ and $G F(N)_{S} \subseteq G(Y) \subseteq G F(E(N))$. If $\eta: G F \longrightarrow 1_{M o d-R}$ is the natural isomorphism, then $N \subseteq \eta(G(Y)) \subseteq E(N)$ and $\eta\left(G(Y) \cong G(Y) \cong K_{R}\right.$. Therefore $N$ is strongly compressible.

Example 3.1.1 An example of a compressible module which is not strongly compressible can be given as follows: Let $R$ be a domain such that $R^{2} \cong R^{3}$ as right $R$-modules. Such a ring $R$ exists by J.D.O'Neill [31]. Clearly $R_{R}$ is compressible, and $\operatorname{dim}\left(R_{R}\right)=\infty$. By Theorem 3.1.1, $R_{R}$ is not strongly compressible.

Corollary 3.1.1 The following are equivalent for a ring $R$ :

(a) $R$ is semiprime right Goldie;

(b) $P_{R}$ is strongly compressible for some progenerator $P_{R}$ of Mod-R;

(c) Every cyclic submodule of some progenerator of Mod-R is strongly compressible; 
(c') Every cyclic essential submodule of some progenerator of Mod- $R$ is strongly compressible;

(d) Every f.g. submodule of some progenerator of Mod-R is strongly compressible;

(d') Every f.g. essential submodule of some progenerator of Mod-R is strongly compressible;

(e) Every submodule of some progenerator of Mod-R is strongly compressible;

(e') Every essential submodule of some progenerator of Mod-R is strongly compressible.

Proof. By Theorem 3.1.1 and Lemmas 3.1.1, 3.1.2 and 3.1.3.

Corollary 3.1.2 $A$ ring $R$ is semiprime right Goldie if and only if $R=$ End $\left(P_{S}\right)$, where $P_{S}$ is a strongly compressible progenerator of Mod-S for some $\operatorname{ring} S$.

\subsection{Some applications}

In this section, using the notion of strongly compressible modules we will present many module-theoretic characterizations of semiprime Goldie (prime right Goldie, or prime Goldie) rings, right $w$-semi-Prüfer (right $w$-Prüfer, semi-Prüfer, or Prüfer) rings as corollaries of Theorem 3.1.1.

Theorem 3.2.1 The following are equivalent for a ring $R$ :

(a) $R$ is semiprime Goldie;

(b) Every f.g. essential submodule of $E\left(R_{R}\right)$ is strongly compressible; 
(c) Every f.g. submodule of $E\left(R_{R}\right)$ is strongly compressible;

(d) $Z\left(R_{R}\right)=0$, and every f.g. nonsingular right $R$-module is strongly compressible.

(e) Every f.g. essential submodule of $E\left(P_{R}\right)$ is strongly compressible for each progenerator $P$ of $M o d-R$;

(f) Every f.g. submodule of $E\left(P_{R}\right)$ is strongly compressible for each progenerator $P$ of Mod-R.

Proof. $(a) \Rightarrow(d)$. Clearly $Z\left(R_{R}\right)=0$. If $M_{R}$ is f.g. nonsingular, then $M$ is embeddable in a f.g. right free $R$-module by Theorem 1.3. Then $M$ is strongly compressible by Theorem 3.1.1.

$(d) \Rightarrow(f)$. This is because for each progenerator $P$ of Mod- $R$, every f.g. submodule of $E\left(P_{R}\right)$ is nonsingular when $R$ is right nonsingular.

$(f) \Rightarrow(e) \Rightarrow(b)$ and $(f) \Rightarrow(c) \Rightarrow(b)$. Obviously.

$(b) \Rightarrow(a)$. By noting that every f.g. essential right ideal of $R$ is essential in $E\left(R_{R}\right)$, we have that $R$ is a semiprime right Goldie ring by Theorem 3.1.1. It is enough to show that $R$ is left Goldie. Let $Q=E\left(R_{R}\right)$. It is well known that $Q$ is a semi-simple Artinian ring and $R$ is a right order of $Q$. Let $x \in Q$. Then $R_{R} \leq_{e} R+x R \subseteq E\left(R_{R}\right)$. Since $R+x R$ is essential in $E\left(R_{R}\right)$, $R+x R$ is strongly compressible, and thus there exists $Y \subseteq E\left(R_{R}\right)$ such that $(R+x R)_{R} \subseteq Y_{R} \stackrel{f}{\cong} R_{R}$. Let $y=f^{-1}(1)$. Then $Y=y R$ and $y^{\perp}=0$, and thus $y$ is a regular element of $Q$. Write $x=y r_{1}, 1=y r_{2}$ for some $r_{i} \in R$. Then $x=r_{2}^{-1} r_{1}$. Hence $R$ is also a left order of $Q$, showing that $R$ is left Goldie. 
Next we show that the characterization theorem of semiprime Goldie rings of López-Permouth, Rizvi and Yousif, which we mentioned in the beginning of this chapter, is a corollary of the previous theorem. To see this we set up a connection between strongly compressible modules and weakly-injective modules which is given by the following proposition. (Comparing it with [26, Prop.3.7].)

Proposition 3.2.1 The following are equivalent for an injective right $R$ module E:

(a) Every submodule of $E$ is weakly-injective;

(b) Every f.g. submodule of $E$ is strongly compressible.

Proof. $(a) \Rightarrow(b)$. Let $N$ be a f.g. submodule of $E$ and $A$ an essential submodule of $N$. Then $E(A)=E(N)$. Since $A$ is weakly-injective, there exists $X \subseteq E(A)=E(N)$ such that $N \subseteq X \cong A$. Thus $N$ is strongly compressible.

$(b) \Rightarrow(a)$. Suppose that $M$ is a submodule of $E$. Let $A$ be a f.g. submodule of $E(M)$. Then $M \cap A$ is essential in $A$. Since $A$ is strongly compressible, there exists a submodule $Y$ of $E(A)$ such that $A \subseteq Y \stackrel{f}{\cong} M \cap A$. Then $f$ induces an isomorphism $E(Y) \stackrel{f}{\cong} E(M \cap A)$. Because $M \cap A$ is essential in $A$ and $A \subseteq Y \subseteq E(A)$, we have $E(Y)=E(A)=E(M \cap A)$. There exists $B \subseteq E(M)$ such that $E(M)=E(A) \oplus B$. If we define $g: E(M) \rightarrow E(M)$ by $g(x+b)=f(x)+b$ for all $x \in E(A)$ and $b \in B$, then $g$ is an $R$-isomorphism and $\left.g\right|_{E(A)}=f$. Let $X=g^{-1}(M)$. Since $f(A) \subseteq M$, we have $A \subseteq X \cong M$ and $X \subseteq E(M)$. Therefore $M$ is weakly-injective. 
Remark 3.2.1 $A$ ring $R$ is called right weakly-semisimple if every right $R$ module is weakly-injective [21]. From the previous proposition, it follows immediately that a ring $R$ is right weakly-semisimple if and only if every f.g. right $R$-module is strongly compressible.

Corollary 3.2.1 [26, Theorem 3.9]. The following are equivalent for a ring $R:$

(a) $R$ is semiprime Goldie;

(b) Every right ideal of $R$ is weakly-injective;

(c) $Z\left(R_{R}\right)=0$ and every nonsingular right $R$-module is weakly-injective.

Proof. Because the class of weakly-injective modules is closed under taking essential extensions, Proposition 3.2.1 implies that $(b)$ is equivalent to $(c)$ of Theorem 3.2.1, and $(c)$ is equivalent to $(d)$ of Theorem 3.2.1.

Proposition 3.2.2 The following are equivalent for a ring $R$ :

(a) $R$ is prime right Goldie;

(b) Every nonzero cyclic right ideal of $R$ is strongly compressible and faithful;

(c) Every nonzero right ideal of $R$ is strongly compressible and faithful;

(d) Every nonzero cyclic submodule of each progenerator of Mod- $R$ is strongly compressible and faithful;

(e) Every nonzero submodule of each progenerator of Mod-R is strongly compressible and faithful.

Proof. By Theorem 3.1.1. 
Strongly compressible modules

Proposition 3.2.3 The following are equivalent for a ring $R$ :

(a) $R$ is prime Goldie;

(b) Every f.g. nonzero submodule of $E\left(R_{R}\right)$ is strongly compressible and faithful;

(c) $Z\left(R_{R}\right)=0$ and every f.g. nonsingular right $R$-module is strongly compressible and faithful.

Proof. By Theorem 3.2.1.

The following are some new characterizations of right $w$-semi-Prüfer (right $w$-Prüfer, semi-Prüfer, or Prüfer) rings.

Proposition 3.2.4 The following are equivalent for a ring $R$ :

(a) $R$ is a right w-semi-Prüfer ring;

(b) Every f.g. essential right ideal of $R$ is a strongly compressible progenerator;

(c) Every f.g. essential submodule of each progenerator of Mod- $R$ is a strongly compressible progenerator.

Proof. By Theorem 3.1.1, Proposition 2.1.1, and Proposition 2.1.3.

Proposition 3.2.5 The following are equivalent for a ring $R$ :

(a) $R$ is a right $w$-Prüfer ring;

(b) Every f.g. nonzero right ideal of $R$ is a strongly compressible progenerator;

(c) Every f.g. nonzero submodule of each progenerator of Mod-R is a strongly compressible progenerator.

Proof. By Theorem 3.1.1, and Proposition 2.1.4. 
Proposition 3.2.6 The following are equivalent for a ring $R$ :

(a) $R$ is a semi-Prüfer ring;

(b) Every f.g. essential submodule of $E\left(R_{R}\right)$ is a strongly compressible progenerator;

(c) Every f.g. essential submodule of $E\left(P_{R}\right)$ is a strongly compressible progenerator for each progenerator $P$ of Mod-R.

Proof. $(c) \Rightarrow(b)$. Obviously.

$(b) \Rightarrow(a)$. By Theorem 3.2.1, Proposition 2.1.1, and noting that every f.g. essential right ideal of $R_{R}$ is essential in $E\left(R_{R}\right)$.

$(a) \Rightarrow(c)$. Let $P$ be a progenerator of $\operatorname{Mod}-R$, and $N$ a f.g. essential submodule of $E\left(P_{R}\right)$. By Theorem 3.2.1, $N$ is strongly compressible. Note that $N \cap P \leq_{e} N$. Thus, there exists $X \subseteq E(N)$ such that $N \subseteq X \cong N \cap P$. Since both $N$ and $P$ have the same finite Goldie dimension, it follows that $N$ can embed in $P$ as an essential submodule. Then $N$ is a progenerator of Mod- $R$ by Proposition 2.1.4.

Proposition 3.2.7 The following are equivalent for a ring $R$ :

(a) $R$ is a Prüfer ring;

(b) Every f.g. nonzero submodule of $E\left(R_{R}\right)$ is a strongly compressible progenerator;

(c) Every f.g. nonzero submodule of $E\left(P_{R}\right)$ is a strongly compressible progenerator for each progenerator $P$ of Mod-R;

(d) $Z\left(R_{R}\right)=0$ and every f.g. nonsingular right $R$-module is a strongly compressible progenerator.

Proof. $(d) \Rightarrow(c) \Rightarrow(b)$. Clearly. 
$(b) \Rightarrow(a)$. By Theorem 3.2.1 and Proposition 2.1.4.

$(a) \Rightarrow(d)$. By Theorem 3.2.1, Proposition 2.1.9, and the fact that every f.g. nonsingular right $R$-module can be embedded in a f.g. free $R$-module.

Proposition 3.2.8 The following are equivalent for a ring $R$ :

(a) $R$ is semi-simple;

(b) Every (right) R-module is strongly compressible;

(c) Every (right) injective R-module is strongly compressible;

(d) $E\left(R_{R}\right)$ is strongly compressible.

Proof. $(a) \Rightarrow(b) \Rightarrow(c) \Rightarrow(d)$. Clearly.

$(d) \Rightarrow(a)$. Since every essential submodule of a strongly compressible module is strongly compressible, it follows from $(d)$ that every f.g. essential submodule of $E\left(R_{R}\right)$ is strongly compressible. Then $R$ is semiprime Goldie by Theorem 3.2.1. On the other hand, condition $(d)$ implies easily that $R \cong E\left(R_{R}\right)$. Thus $R \cong E(R)$ is semi-simple by [18, Th.4.28]. 


\section{Modules over Prüfer rings}

Given a ring $R$, we know that a module $M_{R}$ is projective if and only if $M_{R}$ is a direct summand of some direct sum of copies of $R$. Simply from this, we see that there is a special projective module $R$ which determines the structure of all projective modules. For a commutative Prüfer domain $R$, Fuchs [12] constructed a divisible module $\partial$ with projective dimension at most one which functions as $R$ in the sense that a module $M_{R}$ is divisible with projective dimension at most one if and only if $M$ is a direct summand of some direct sum of copies of $\partial$. In this chapter, we will extend this result to a noncommutative Prüfer ring. This work is carried out in Section 2. In Section 1, we establish a structure theorem for modules of projective dimension one over a noncommutative Prüfer ring. Besides its own interest, the structure theorem is also needed for the proof of the above-mentioned result.

\subsection{Modules of projective dimension at most one}

First let us recall some concepts in Module Theory. For a fixed module $M_{R}$, $\operatorname{Ext}^{n}(M,-)$ is the $n$th right derived functor of $\operatorname{Hom}(M,-)$. If $0 \rightarrow A \rightarrow$ $B \rightarrow C \rightarrow 0$ is a short exact sequence of right $R$-modules, then we have the long exact sequence in the second variable

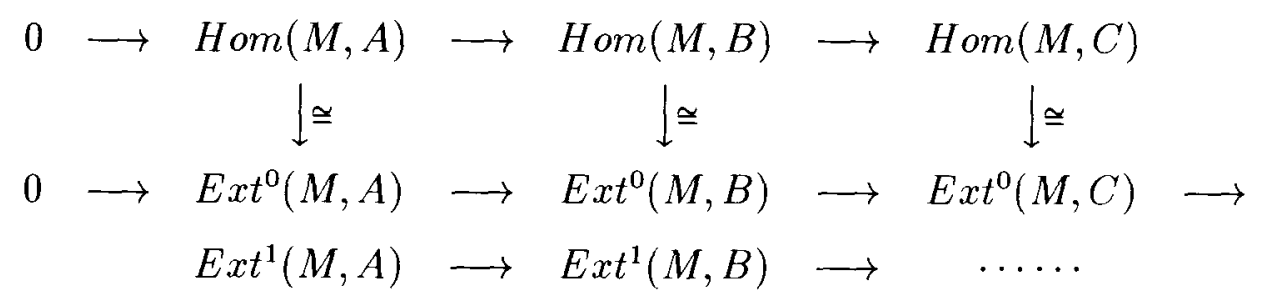


Similarly, $\operatorname{Ext}^{n}(-, M)$ is the $n$th right derived functor of $\operatorname{Hom}(-, M)$. And it induces the long exact sequence in the first variable. A basic fact of the Ext functor is that $\operatorname{Ext}_{R}^{1}(M, N)=0$ if and only if any exact sequence $0 \rightarrow N \rightarrow D \rightarrow M \rightarrow 0$ splits.

The projective dimension of a module $M_{R}$, denoted by $\operatorname{Pd}\left(M_{R}\right)$ or simply by $\operatorname{Pd}(M)$, is the smallest nonnegative integer $n$ such that $\operatorname{Ext}^{n+1}(M, N)=0$ for all $N \in \operatorname{Mod}-R$, if such an integer $n$ exists. If no such $n$ exists, then $\operatorname{Pd}\left(M_{R}\right)=\infty$. Also, $\operatorname{Pd}\left(M_{R}\right)=n$ if and only if for any projective resolution of $M_{R}$ :

$$
\cdots \rightarrow P_{n} \stackrel{d_{n}}{\rightarrow} P_{n-1} \rightarrow \cdots \rightarrow P_{1} \stackrel{d_{1}}{\rightarrow} P_{0} \stackrel{d_{9}}{\rightarrow} M \rightarrow 0,
$$

$\operatorname{Im}\left(d_{n}\right)$ is projective [22, P90]. Clearly, $P d\left(M_{R}\right)=0$ if and only if $M$ is projective. If $0 \rightarrow A \rightarrow B \rightarrow C \rightarrow 0$ is a short exact sequence of right $R$-modules with $B$ projective, then, by examining the induced long exact sequence in the second variable, we have $P d(A)=P d(C)-1$.

Lemma 4.1.1 If $M_{R}$ is finitely generated and $R$ is a Prüfer ring, then $M \cong$ $\tau(M) \oplus M / \tau(M)$.

Proof. Since $M / \tau(M)$ is f.g. torsionfree, then it is projective by Proposition 2.3.5. Therefore the short exact sequence $0 \rightarrow \tau(M) \rightarrow M \rightarrow M / \tau(M) \rightarrow 0$ splits, and so $M \cong \tau(M) \oplus M / \tau(M)$.

For some ordinal $\rho$, let

$$
0=M_{0} \subseteq M_{1} \subseteq \cdots \subseteq M_{\alpha} \subseteq \cdots \subseteq M_{\rho}=M(\alpha<\rho)
$$

be a well-ordered ascending chain of submodules of a module $M_{R}$. The chain (1) is said to be continuous if $M_{\beta}=\bigcup_{\alpha<\beta} M_{\alpha}$ for every limit ordinal $\beta \leq \rho$. 
Lemma 4.1.2 [Auslander]. For an ordinal $\rho$, let

$$
0=M_{0} \subseteq M_{1} \subseteq \cdots \subseteq M_{\alpha} \subseteq \cdots(\alpha<\rho)
$$

be a well-ordered ascending chain of submodules of a module $M_{R}$ such that

(a) $\bigcup_{\alpha<\rho} M_{\alpha}=M$;

(b) (1) is a continuous chain;

(c) $\operatorname{Pd}\left(M_{\alpha+1} / M_{\alpha}\right) \leq n$ for some fixed integer $n$ and all $1 \leq \alpha+1<\rho$.

Then $\operatorname{Pd}(M) \leq n$.

Proof. If $n=0$, then, since $(b), M_{\alpha} /\left(\cup_{\sigma<\alpha} M_{\sigma}\right)$ is projective for all $\alpha<\rho$. It follows that $M_{\alpha}=\left(\cup_{\sigma<\alpha} M_{\sigma}\right) \oplus M_{\alpha}^{\prime}$ for some projective submodule $M_{\alpha}^{\prime}$ of $M$. Therefore $M=\cup_{\alpha<\rho} M_{\alpha}=\oplus_{\alpha<\rho} M_{\alpha}^{\prime}$ is projective, thus $\operatorname{Pd}(M)=0$. Now assume $n>0$. Let $M_{\alpha}^{\prime}=M_{\alpha} /\left(\cup_{\sigma<\alpha} M_{\sigma}\right)$, and $F_{\alpha}^{\prime}$ be a free right $R$-module mapping onto $M_{\alpha}^{\prime}$ with kernel $K_{\alpha}^{\prime}$. If $\alpha$ is a limit ordinal, then $M_{\alpha}^{\prime}=0$. In this case we choose 0 as $F_{\alpha}^{\prime}$. Therefore we have $\operatorname{Pd}\left(K_{\alpha}^{\prime}\right)=\operatorname{Pd}\left(M_{\alpha}^{\prime}\right)-1 \leq n-1$. Let $F_{\alpha}=\oplus_{\sigma \leq \alpha} F_{\sigma}^{\prime}$. Since $F_{\alpha}^{\prime}$ is free, there is a map $F_{\alpha}^{\prime} \rightarrow M_{\alpha}$ which lifts the map $F_{\alpha}^{\prime} \rightarrow M_{\alpha}^{\prime}$. By transfinite induction, the map $F_{\alpha}^{\prime} \rightarrow M_{\alpha}$ can be extended to a map $F_{\alpha} \rightarrow M_{\alpha}$ such that if $K_{\alpha}$ is the kernel, then $K_{\sigma} \subseteq K_{\alpha}$ for $\sigma<\alpha$ and $K_{\alpha}^{\prime} \cong K_{\alpha} /\left(\cup_{\sigma<\alpha} K_{\sigma}\right)$. Thus, $\operatorname{Pd}\left(K_{\alpha} /\left(\cup_{\sigma<\alpha} K_{\sigma}\right)\right)=\operatorname{Pd}\left(K_{\alpha}^{\prime}\right) \leq n-1$. Note that $0=K_{0} \subseteq K_{1} \subseteq \cdots \subseteq K_{\alpha} \subseteq \cdots$ is a continuous chain. By the induction hypothesis, $\operatorname{Pd}\left(\cup_{\alpha<\rho} K_{\alpha}\right) \leq n-1$. Since $\cup_{\alpha<\rho} K_{\alpha}$ is the kernel of $\cup_{\alpha<\rho} F_{\alpha} \rightarrow M$, and $\cup_{\alpha<\rho} F_{\alpha}=\oplus_{\alpha<\rho} F_{\alpha}^{\prime}$ is projective, we obtain $P d(M) \leq n$.

A module $M_{R}$ is finitely presented if there is an exact sequence $0 \rightarrow K \rightarrow$ $R^{n} \rightarrow M \rightarrow 0$, where $n$ is a positive integer and $K$ is finitely generated. This is equivalent to the requirement that there exist f.g. modules $K_{R}$ and $P_{R}$ 
such that $0 \rightarrow K \rightarrow P \rightarrow M \rightarrow 0$ is exact (see [2, Ex.17, P233]).

Lemma 4.1.3 Let $R$ be a Prüfer ring. A f.g. module $M_{R}$ is finitely presented if and only if $P d(M) \leq 1$.

Proof. Let $0 \rightarrow H \rightarrow F \rightarrow M \rightarrow 0$ be an exact sequence with $F$ f.g. free. If $M$ is finitely presented, then $H$ is finitely generated. Hence $H$ is f.g. torsionfree. By Proposition 2.3.5, $H$ is projective. Therefore $P d(M) \leq 1$.

Conversely, if $\operatorname{Pd}(M) \leq 1$, then $H$ is projective. Since $H \subseteq F$ and $F$ has finite Goldie dimension, $H$ is of finite Goldie dimension. By Proposition 2.3.5, $H$ is finitely generated.

Lemma 4.1.4 Let $R$ be a Prüfer ring, and $H$ a projective submodule of a torsionfree module $F_{R}$. If $F / H$ is finitely generated, then $F$ is projective and $F / H$ is finitely presented.

Proof. Step 1. First we assume $R$ is a Prüfer domain (noncommutative), $H$ is free and $F / H$ is f.g. torsion. Write $H=\bigoplus\{y R: y \in Y\}$. Let $Q=Q_{c l}(R)$. Then $Q$ is a division ring. Since $F$ is torsionfree, the map $\psi: F \rightarrow F \otimes \otimes_{R} Q$ which is defined by $\psi(x)=x \otimes 1$ is one to one. Since $F / H$ is torsion, and $Y$ is a basis for $H,\{y \otimes 1: y \in Y\}$ becomes a basis for the $Q$-vector space $\left(F \otimes_{R} Q\right)_{Q}$. Suppose $F / H=\overline{x_{1}} R+\cdots+\overline{x_{m}} R$, where $\overline{x_{i}}=x_{i}+H, i=1, \ldots, m$. Clearly $F=x_{1} R+\cdots+x_{m} R+H$. For each $i$, there exists a nonzero $r_{i}$ of $R$ such that $x_{i} r_{i} \in y_{1} R+\cdots+y_{k} R$, where $k$ is a fixed positive integer. Let $H_{0}=y_{1} R+\cdots+y_{k} R, F_{0}=x_{1} R+\cdots+x_{m} R+H_{0}$. Then the map $\varphi: F_{0} / H_{0} \rightarrow F / H$ defined by $\varphi\left(\xi+H_{0}\right)=\xi+H$ is onto.

Claim: $F_{0} \cap H=H_{0}$. Let $\xi=x_{1} a_{1}+\cdots+x_{m} a_{m} \in H$, where each $a_{i} \in R$. Write $\xi=y_{i_{1}} b_{1}+\cdots+y_{i_{n}} b_{n}$ for some $y_{i_{j}} \in Y$ and $0 \neq b_{i} \in R$. 
Then $\xi \otimes 1=\left(y_{i_{1}} \otimes 1\right) b_{1}+\cdots+\left(y_{i_{n}} \otimes 1\right) b_{n}$. On the other hand, $\xi \otimes 1=$ $\left(x_{1} \otimes 1\right) a_{1}+\cdots+\left(x_{m} \otimes 1\right) a_{m}=\left(x_{1} r_{1} \otimes 1\right) r_{1}^{-1} a_{1}+\cdots+\left(x_{m} r_{m} \otimes 1\right) r_{m}^{-1} a_{m}=\left(y_{1} \otimes\right.$ 1) $u_{1}+\cdots+\left(y_{k} \otimes 1\right) u_{k}$ for some $u_{k} \in Q$, since each $x_{i} r_{i} \in y_{1} R+\cdots+y_{k} R$. By noting that each $b_{i} \neq 0$, and $\{y \otimes 1: y \in Y\}$ is a basis of $\left(F \otimes \otimes_{R} Q\right)_{Q}$, we have $\left\{y_{i_{1}}, \ldots, y_{i_{n}}\right\} \subseteq\left\{y_{1}, \ldots, y_{k}\right\}$. Therefore $\xi \in H_{0}$. Consequently $F_{0} \cap H=H_{0}$, implying that $\varphi$ is an isomorphism. Since both $H_{0}$ and $F_{0}$ are f.g. torsionfree, they are f.g. projective by Proposition 2.3.5. Therefore $F_{0} / H_{0}$, and $F / H$ is finitely presented. We note that $H_{0}$ is a direct summand of $H_{R}$, hence $H / H_{0}$ is projective. Since $F=x_{1} R+\cdots+x_{m} R+H$ and $F_{0} \cap H=H_{0}$, we have an $R$-module isomorphism $\theta: H / H_{0} \rightarrow F / F_{0}$ (via $\left.\theta\left(x+H_{0}\right)=x+F_{0}\right)$. Therefore $F / F_{0}$ is projective. So we have $F \cong F_{0} \oplus\left(F / F_{0}\right)$ and $F$ is projective.

Step 2. Assume $R$ is a Prüfer domain, $H$ is projective and $F / H$ is f.g. torsion. Then $H \oplus X$ is free for some $X \in \operatorname{Mod}-R$. $X$ is, of course, torsionfree. Therefore $F \oplus X$ is torsionfree and

$$
0 \rightarrow H \oplus X \rightarrow F \oplus X \rightarrow(F \oplus X) /(H \oplus X) \cong F / H \rightarrow 0
$$

is exact. Step 1 implies that $(F \oplus X) /(H \oplus X)$ is finitely presented and $F \oplus$ $X$ is projective. Consequently $F / H$ is finitely presented and $F$ is projective.

Step 3. We assume $R$ is a Prüfer domain, $H$ is projective and $F / H$ is finitely generated. By Lemma 4.1.1, we may assume $F / H=(U / H) \oplus(V / H)$ where $U / H=\tau(F / H)$ is f.g. torsion, and $V / H \cong(F / H) / \tau(F / H)$ is f.g. torsionfree. By Proposition 2.3.5, $V / H$ is projective. Therefore we have $V \cong H \oplus(V / H)$, and so $V$ is projective. In the following short exact sequence:

$$
0 \rightarrow V \rightarrow F \rightarrow F / V \cong U / H \rightarrow 0
$$


$U / H$ is f.g. torsion, and $V$ is projective. Therefore Step 2 implies that $F$ is projective and $U / H$ is finitely presented. As a direct sum of two finitely presented modules, $F / H$ is of course finitely presented.

Step 4. The general case: $R$ is a Prüfer ring. We know that $R$ is Morita equivalent to a Prüfer domain $S$ by Theorem 2.3.5. There exists a Morita equivalence $G: \operatorname{Mod}-R \rightarrow \operatorname{Mod}-S$, and $G$ induces an exact sequence in Mod-S:

$$
0 \rightarrow G(H) \rightarrow G(F) \rightarrow G(F / H) \rightarrow 0
$$

By [2, Prop.21.6], $G(H)_{S}$ is projective, and $G(F / H)_{S}$ is finitely generated. It is well-known that the singularity of modules is preserved under Morita equivalences (e.g., see [17, P43]). Then the torsionfreeness of $F_{R}$ implies that $G(F)_{S}$ is torsionfree. Therefore Step 3 implies that $G(F)_{S}$ is projective and $G(F / H)_{S}$ is finitely presented. Hence $F_{R}$ is projective and $(F / H)_{R}$ is finitely presented by [2, Ex.11, P262].

A right $R$-module is called coherent, if every f.g. submodule is finitely presented.

Proposition 4.1.1 Every module $M_{R}$ of projective dimension 1 over a Prüfer ring $R$ is coherent, and for any submodule $N$ of $M, \operatorname{Pd}(N) \leq 1$ and $\operatorname{Pd}(M / N) \leq$ 1.

Proof. Let $N$ be a f.g. submodule of $M_{R}$ where $\operatorname{Pd}(M)=1$. Then we can write $M \cong F / H$ with $F$ free and $H$ projective. There exists a submodule $G$ of $F_{R}$ such that $H \subseteq G$ and $N \cong G / H$. Clearly $G$ is torsionfree. Therefore $N$ is finitely presented, and $G$ is projective by Lemma 4.1.4. Since $0 \rightarrow G \rightarrow$ $F \rightarrow F / G \cong M / N \rightarrow 0$ is exact, we have $\operatorname{Pd}(M / N) \leq 1$. 
Theorem 4.1.1 Over a Prüfer ring, a countably generated right module has projective dimension $\leq 1$ if and only if it is the union of a countable ascending chain of finitely presented right modules.

Proof. Given a countably generated module $M_{R}$, then $M_{R}$ is a union of a countable ascending chain of f.g. submodules. If $\operatorname{Pd}(M)=0$, i.e., $M$ is projective, then every f.g. submodule of $M$ is torsionfree, and hence is projective by Proposition 2.3.5, and hence finitely presented by Lemma 4.1.3. If $\operatorname{Pd}(M)=1$, then, by Proposition 4.1.1, every f.g. submodule of $M$ is finitely presented. For the converse, we suppose $M_{R}$ is the union of a chain of right finitely presented $R$-modules:

$$
0 \subseteq M_{1} \subseteq M_{2} \subseteq \cdots \subseteq M_{n} \subseteq \cdots
$$

By [2, Ex.17, P233], all $M_{n+1} / M_{n}$ are finitely presented. Then Lemma 4.1.3 implies that $\operatorname{Pd}\left(M_{n+1} / M_{n}\right) \leq 1$ for all $n$. By Lemma 4.1.2, $\operatorname{Pd}(M) \leq 1$.

Let $R$ be a Prüfer domain, and $0 \rightarrow H \rightarrow F \stackrel{\phi}{\rightarrow} M \rightarrow 0$ be an exact sequence of right $R$-modules such that $F_{R}=\bigoplus\{x R: x \in X\}$ is free on $X$ and $H$ is projective. By [2, Cor.26.2], $H_{R}=\bigoplus\left\{H_{y}: y \in Y\right\}$, where the $H_{y}$ 's are countably generated projective right $R$-modules. Consider all pairs $\left(X_{i}, Y_{i}\right)$ of subsets $X_{i} \subseteq X, Y_{i} \subseteq Y$ such that $F_{i}=\bigoplus\left\{x R: x \in X_{i}\right\}$ and $H_{i}=\bigoplus\left\{H_{y}: y \in Y_{i}\right\}$ satisfy $H_{i}=H \cap F_{i}$. Let $i$ run over an index set $I$. Note that $H=H_{i} \oplus H_{i}^{*}$ and $F_{i}+H=F_{i} \oplus H_{i}^{*}$, where $H_{i}^{*}=\bigoplus\left\{H_{y}: y \in\right.$ $\left.Y \backslash Y_{i}\right\}$. Therefore each $F_{i}+H$ is projective. Set $\mathcal{T}=\left\{M_{i}: i \in I\right\}$, where $M_{i}=\left(F_{i}+H\right) / H$. Then, clearly, $(0), M \in \mathcal{T}$, and for $M_{i}, M_{j} \in \mathcal{T}$ with $M_{i} \subset M_{j}, M_{j} / M_{i} \cong\left(F_{j}+H\right) /\left(F_{i}+H\right)$ has projective dimension at most one. 
Lemma 4.1.5 [Fuchs]. Let $R, M_{R}$, and $\mathcal{T}$ be as above. Then for any countable subset $\triangle$ of $M$, there exists some $M_{i} \in \mathcal{T}$ with $M_{i}$ countably generated such that $\langle\triangle\rangle \subseteq M_{i}$, where $\langle\triangle\rangle$ indicates the submodule of $M$ generated by $\triangle$.

Proof. Given a countable subset $\triangle$ of $M$, there is a countable subset $X^{(1)}$ of $X$ such that $\phi\left\langle X^{(1)}\right\rangle$ contains $\triangle$. Let $Q=Q_{c l}(R)$. Then $Q$ is a division ring. Since $\left\langle X^{(1)}\right\rangle$ is torsionfree, we have that $f:\left\langle X^{(1)}\right\rangle \rightarrow\left\langle X^{(1)}\right\rangle \otimes_{R} Q$ which is defined by $f(a)=a \otimes 1$ is one to one. Similarly, $g:\left\langle X^{(1)}\right\rangle \cap H \longrightarrow$ $\left(\left\langle X^{(1)}\right\rangle \cap H\right) \otimes_{R} Q(g(b)=b \otimes 1)$ is one to one. Since $Q$ is a flat left $R$-module, the map $l \otimes 1:\left(\left\langle X^{(1)}\right\rangle \cap H\right) \otimes_{R} Q \longrightarrow\left\langle X^{(1)}\right\rangle \otimes_{R} Q$ is a monomorphism, where $l$ is the inclusion of $\left\langle X^{(1)}\right\rangle \cap H$ into $\left\langle X^{(1)}\right\rangle$. Therefore we have the following commutative diagram:

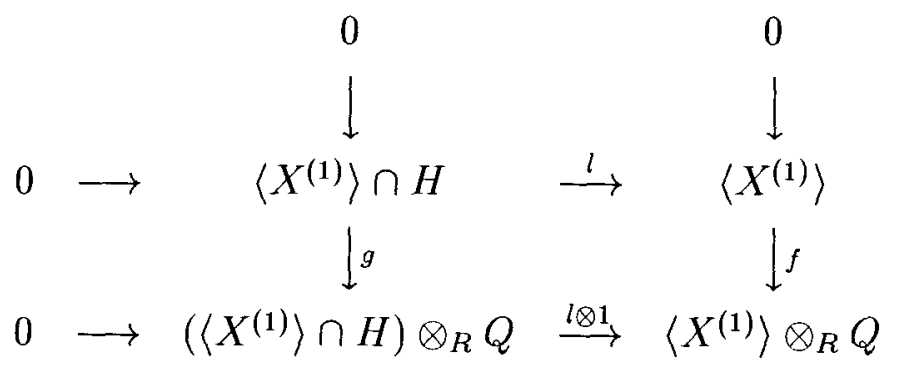

Clearly, $l \otimes 1$ is a $Q$-homomorphism. Since $\left\langle X^{(1)}\right\rangle$ is free with a basis $X^{(1)}$, $\left\langle X^{(1)}\right\rangle \otimes_{R} Q$ is a $Q$-vector space with a basis $\left\{x \otimes 1: x \in X^{(1)}\right\}$. Thus, as a $Q$-subspace, $\left(\left\langle X^{(1)}\right\rangle \cap H\right) \otimes_{R} Q$ has a countable basis which, we may assume, is $\left\{z_{i} \otimes 1: i \in \mathbf{N}\right\}$ with all $z_{i} \in\left\langle X^{(1)}\right\rangle \cap H$. There is a countable subset $Y^{(1)}$ of $Y$ such that all $z_{i} \in \oplus_{y \in Y^{(1)}} H_{y}$. We claim that $\left\langle X^{(1)}\right\rangle \cap H \subseteq \oplus_{y \in Y^{(1)}} H_{y}$. In fact, if not, then we can find an $h=h_{a}+h_{b} \in\left\langle X^{(1)}\right\rangle \cap H$ with $0 \neq h_{a} \in$ 
$\oplus_{y \in Y \backslash Y^{(1)}} H_{y}$, and $h_{b} \in \oplus_{y \in Y^{(1)}} H_{y}$. But since $h \otimes 1 \in\left(\left\langle X^{(1)}\right\rangle \cap H\right) \otimes_{R} Q, h \otimes 1=$ $\sum_{i=1}^{n}\left(z_{i} \otimes 1\right) q_{i}$ for some $q_{i} \in Q$. There exist $a_{i} \in R$ and $c \in \mathcal{C}_{R}(0)$ such that $q_{i}=a_{i} c^{-1}$ for $i=1, \cdots, n$. Then $h c \otimes 1=(h \otimes 1) c=\left(\sum_{i=1}^{n} z_{i} a_{i}\right) \otimes 1$. It follows that $h c=\sum_{i=1}^{n} z_{i} a_{i}$. This implies that $h_{a} c=0$, contradicting the fact that $H$ is torsionfree. Hence the claim is true. We can select a countable subset $X^{(2)}$ of $X$ that contains $X^{(1)}$ and satisfies $\oplus_{y \in Y^{(1)}} H_{y} \subseteq\left\langle X^{(2)}\right\rangle$. Repeating this process, we obtain ascending chains of countable subsets

$$
X^{(1)} \subseteq X^{(2)} \subseteq \cdots \subseteq X^{(n)} \subseteq \cdots
$$

and

$$
Y^{(1)} \subseteq Y^{(2)} \subseteq \cdots \subseteq Y^{(n)} \subseteq \cdots
$$

of $X$ and $Y$, respectively, such that

$$
\left\langle X^{(n)}\right\rangle \cap H \subseteq \oplus_{y \in Y^{(n)}} H_{y} \subseteq\left\langle X^{(n+1)}\right\rangle
$$

for each $n \leq 1$. Let $X^{*}=\cup_{n} X^{(n)}, Y^{*}=\cup_{n} Y^{(n)}, F^{*}=\oplus\left\{R x: x \in X^{*}\right\}$, and $H^{*}=\oplus\left\{H_{y}: y \in Y^{*}\right\}$. Then $F^{*} \cap H=H^{*}$. Thus $M^{*}=\left(F^{*}+H\right) / H \in \mathcal{T}$. It is clear that $\langle\triangle\rangle \subseteq M^{*}$, and $M^{*}$ is countably generated.

Lemma 4.1.6 Let $R, M_{R}$, and $\mathcal{T}$ be as above. Given $M_{i}=\left(F_{i}+H\right) / H \in \mathcal{T}$ and a countable subset $\triangle$ of $M$, there exists some $M_{j}=\left(F_{j}+H\right) / H \in \mathcal{T}$ such that $\left\langle\triangle, M_{i}\right\rangle \subseteq M_{j}, M_{j} / M_{i}$ is countably generated, and $F_{i} \subseteq F_{j}$.

Note. The required condition $F_{i} \subseteq F_{j}$ is really indispensable for the proof of the next lemma.

Proof. We consider the following short exact sequence:

$$
0 \rightarrow\left(F_{i}+H\right) / F_{i} \rightarrow F / F_{i} \rightarrow\left(F / F_{i}\right) /\left[\left(F_{i}+H\right) / F_{i}\right] \rightarrow 0 .
$$

Clearly

$$
\left(F / F_{i}\right) /\left[\left(F_{i}+H\right) / F_{i}\right] \cong F /\left(F_{i}+H\right)
$$


has projective dimension at most one,

$$
F / F_{i}=\oplus\left\{\bar{x} R: x \in X \backslash X_{i}\right\}
$$

is free, where

$$
\bar{x}=x+F_{i} \in F / F_{i},
$$

and

$$
\left(F_{i}+H\right) / F_{i}=\oplus\left\{\left(H_{y}+F_{i}\right) / F_{i}: y \in Y \backslash Y_{i}\right\}\left(\cong H_{i}^{*}\right)
$$

is projective with each

$$
\left(H_{y}+F_{i}\right) / F_{i} \cong H_{y}
$$

countably generated. By Lemma 4.1.5, there exist

$$
X^{\prime} \subseteq X \backslash X_{i}, Y^{\prime} \subseteq Y \backslash Y_{i}
$$

such that:

(a) $\oplus\left\{\bar{x} R: x \in X^{\prime}\right\} \cap\left(\left(F_{i}+H\right) / F_{i}\right)=\oplus\left\{\left(H_{y}+F_{i}\right) / F_{i}: y \in Y^{\prime}\right\}$,

i.e.,

$$
\left(\oplus\left\{x R: x \in X^{\prime}\right\} \cap\left(F_{i}+H\right)\right)+F_{i}=\oplus\left\{H_{y}: y \in Y^{\prime}\right\}+F_{i} ;
$$

and

(b) $\left[\oplus\left\{\bar{x} R: x \in X^{\prime}\right\}+\left(\left(F_{i}+H\right) / F_{i}\right)\right] /\left[\left(F_{i}+H\right) / F_{i}\right]$

is countably generated; and

(c) $\left[\oplus\left\{\bar{x} R: x \in X^{\prime}\right\}+\left(\left(F_{i}+H\right) / F_{i}\right)\right] /\left[\left(F_{i}+H\right) / F_{i}\right] \supseteq\left\langle\overline{\overline{\triangle_{F}}}\right\rangle$, where $\triangle_{F}$ is a countable subset of $F$ such that

$$
\triangle=\left\{u+H: u \in \triangle_{F}\right\}, \overline{\triangle_{F}}=\left\{v+F_{i}: v \in \triangle_{F}\right\},
$$

and

$$
\overline{\overline{\triangle_{F}}}=\left\{f+\left[\left(F_{i}+H\right) / F_{i}\right]: f \in \overline{\triangle_{F}}\right\} .
$$

It is easy to see that condition $(c)$ is equivalent to

$$
\oplus\left\{x R: x \in X^{\prime}\right\}+F_{i}+H \supseteq \sum_{u \in \Delta_{F}} u R+F_{i}+H .
$$

Let 


$$
\begin{gathered}
F^{\prime \prime}=\bigoplus\left\{x R: x \in X^{\prime} \cup X_{i}\right\}, \\
H^{\prime \prime}=\bigoplus\left\{H_{y}: y \in Y^{\prime} \cup Y_{i}\right\}, \\
M^{\prime \prime}=\left(F^{\prime \prime}+H\right) / H .
\end{gathered}
$$

Then, by $(*)$,

$$
F^{\prime \prime} \cap H=\left(\oplus\left\{x R: x \in X^{\prime}\right\} \oplus F_{i}\right) \cap H \supseteq\left(\oplus\left\{H_{y}: y \in Y^{\prime}\right\}\right) \oplus H_{i}=H^{\prime \prime} .
$$

On the other hand, if

$$
b \in F^{\prime \prime} \cap H \text {, i.e., } b=b_{1}+b_{2} \in H
$$

for some

$$
b_{1} \in \bigoplus\left\{x R: x \in X^{\prime}\right\}, b_{2} \in F_{i}
$$

then

$$
b_{1}=b-b_{2} \in \bigoplus\left\{x R: x \in X^{\prime}\right\} \cap\left(F_{i}+H\right) .
$$

By $(*), b-b_{2}=a_{1}+a_{2}$ for some

$$
a_{1} \in \bigoplus\left\{H_{y}: y \in Y^{\prime}\right\}, a_{2} \in F_{i} .
$$

Then

$$
b-a_{1}=a_{2}+b_{2} \in F_{i} \cap H=H_{i} .
$$

Therefore

$$
b=a_{1}+\left(a_{2}+b_{2}\right) \in\left\{H_{y}: y \in Y^{\prime}\right\}+H_{i}=H^{\prime \prime} .
$$

Consequently we have

$$
F^{\prime \prime} \cap H=H^{\prime \prime} \text {, and hence } M^{\prime \prime} \in \mathcal{T} \text {. }
$$

Also, by $(* *)$,

$$
\begin{aligned}
& \left\langle\triangle, M_{i}\right\rangle=\left(\sum_{u \in \triangle_{F}} u R+H\right) / H+\left(F_{i}+H\right) / H= \\
& \left(\sum_{u \in \triangle_{F}} u R+F_{i}+H\right) / H \subseteq\left(F^{\prime \prime}+H\right) / H=M^{\prime \prime} .
\end{aligned}
$$

Clearly $F_{i} \subseteq F^{\prime \prime}$. Finally

$$
M^{\prime \prime} / M_{i} \cong\left[\bigoplus\left\{\bar{x} R: x \in X^{\prime}\right\}+\left(\left(F_{i}+H\right) / F_{i}\right)\right] /\left[\left(F_{i}+H\right) / F_{i}\right]
$$

is countably generated by $(b)$. The proof is complete. 
Now we can prove the following lemma:

Lemma 4.1.7 Let $R$ be a Prüfer ring and $\operatorname{Pd}(M) \leq 1$. Then there exists a well-ordered continuous chain of submodules

$$
0=M_{0} \subset M_{1} \subset \cdots \subset M_{\alpha} \subset \cdots \subset M_{p}=M(\alpha<\rho)
$$

such that for each $\alpha<\rho, M_{\alpha+1} / M_{\alpha}$ is finitely presented.

Proof. Step 1. We assume $R$ is a Prüfer domain. Then we can set up $\mathcal{T}$ as in the above discussion. Choose $M_{0}=(0) \in \mathcal{T}$. Suppose we have already chosen all $M_{\alpha}=\left(F_{\alpha}+H\right) / H$ for all $\alpha<\sigma$ with $M_{\alpha} \in \mathcal{T}$ such that $0 \neq M_{\alpha+1} / M_{\alpha}$ is countably generated and $F_{\alpha} \subseteq F_{\alpha+1}$ for all $\alpha+1<\sigma$.

(i) $\sigma$ is not a limit ordinal. We are done if $M=M_{\sigma-1}$. If $M \neq M_{\sigma-1}$, then, by Lemma 4.1.6, there exists some $M_{\sigma} \in \mathcal{T}$ such that $M_{\sigma-1} \subset M_{\sigma}$, $M_{\sigma} / M_{\sigma-1}$ is countably generated, and $F_{\sigma-1} \subseteq F_{\sigma}$.

(ii) $\sigma$ is a limit ordinal. We can define $M_{\sigma}=\bigcup_{\alpha<\sigma} M_{\alpha}$. Let $F_{\sigma}=$ $\sum_{\alpha<\sigma} F_{\alpha}, H_{\sigma}=\bigoplus\left\{H_{y}: y \in \bigcup_{\alpha<\sigma} Y_{\alpha}\right\}$. Then $F_{\sigma}=\bigoplus\left\{x R: x \in \bigcup_{\alpha<\sigma} X_{\alpha}\right\}$, and $F_{\sigma} \cap H=H_{\sigma}$ since $\left\{F_{\alpha}\right\}$ is a chain. Therefore $M_{\sigma}=\sum_{\alpha<\sigma} M_{\alpha}=$ $\left(F_{\sigma}+H\right) / H \in \mathcal{T}$. Note that $0 \neq M_{\alpha+1} / M_{\alpha}$ is countably generated for all $\alpha<\sigma$. By transfinite induction, we can get a continuous chain of submodules of $M_{R}$ from $\mathcal{T}$ :

$$
0=M_{0} \subset M_{1} \subset \cdots \subset M_{\alpha} \subset \cdots \subset M_{\rho}=M
$$

such that $M_{\alpha+1} / M_{\alpha}$ is countably generated for all $\alpha<\rho$. From the notes before Lemma 4.1.5, each $\operatorname{Pd}\left(M_{\alpha+1} / M_{\alpha}\right) \leq 1$. Then, for each $\alpha$, Theorem 4.1.1 ensures that there exists a chain of submodules 


$$
M_{\alpha}=M_{\alpha}^{0} \subset M_{\alpha}^{1} \subset \cdots \subset M_{\alpha}^{\aleph^{0}}=M_{\alpha+1}
$$

such that $M_{\alpha}^{i+1} / M_{\alpha}^{i}$ is finitely presented for all $i$. Therefore, without loss of generality, we may assume each $M_{\alpha+1} / M_{\alpha}$ is finitely presented.

Step 2. Let $R$ be a Prüfer ring. Then, by Theorem 2.3.5, $R$ is Morita equivalent to a Prüfer domain $S$ via an equivalence $F: \operatorname{Mod}-R \rightarrow \operatorname{Mod}-S$ with inverse $G: \operatorname{Mod}-S \rightarrow \operatorname{Mod}-R$. Since $P d(M) \leq 1$, then $P d(F(M)) \leq 1$. By Step 1, there exists a continuous chain of submodules of $F(M)_{S}$ :

$$
0=N_{0} \subset N_{1} \subset \cdots \subset N_{\alpha} \subset \cdots \subset N_{\rho}=F(M)
$$

such that $N_{\alpha+1} / N_{\alpha}$ is finitely presented for all $\alpha<\rho$. Since Morita equivalence preserves exactness [2, Prop.21.4], we have $G\left(N_{\alpha+1}\right) / G\left(N_{\alpha}\right) \cong G\left(N_{\alpha+1} / N_{\alpha}\right)$. It follows from $\left[2\right.$, Ex.11, P262] that $G\left(N_{\alpha+1}\right) / G\left(N_{\alpha}\right)$ is finitely presented for each $\alpha<\rho$. If $N_{\sigma}=\bigcup_{\alpha<\sigma} N_{\sigma}$, then $G\left(N_{\alpha}\right)=\bigcup_{\alpha<\sigma} G\left(N_{\sigma}\right)$ by [2, Prop.21.7]. Therefore we have shown

$$
0=G\left(N_{0}\right) \subset G\left(N_{1}\right) \subset \cdots \subset G\left(N_{\alpha}\right) \subset \cdots \subset G\left(N_{\rho}\right)=G F(M)_{R}
$$

is a continuous chain of submodules of $G F(M)_{R}$ such that $G\left(N_{\alpha+1}\right) / G\left(N_{\alpha}\right)$ is finitely presented for all $\alpha<\rho$. Since $M_{R} \cong G F(M)_{R}$, we can get such a similar chain for $M_{R}$.

Now we can prove the main theorem of this section.

Theorem 4.1.2 Let $M_{R}$ be a module over a Prüfer ring $R$. Then $\operatorname{Pd}(M) \leq$ 1 if and only if $M$ is the union of a well-ordered continuous chain of submodules

$$
0=M_{0} \subset M_{1} \subset \cdots \subset M_{\alpha} \subset \cdots \subset M_{\rho}=M
$$


such that $M_{\alpha+1} / M_{\alpha}$ is finitely presented cyclic for all $\alpha<\rho$.

Proof. The sufficiency follows from Lemmas 4.1.2, 4.1.3. For the necessity, we know that there is a well-ordered continuous chain of submodules

$$
0=M_{0} \subset M_{1} \subset \cdots \subset M_{\alpha} \subset \cdots \subset M_{\rho}=M
$$

such that $M_{\alpha+1} / M_{\alpha}$ is finitely presented for all $\alpha<\rho$, by Lemma 4.1.7. Therefore, to complete the proof, it suffices to show the fact that for every finitely presented module $N_{R}$, there exists a finite chain of submodules of $N_{R}$ such that each factor of this chain is finitely presented cyclic. To see this, let $N=x_{1} R+\cdots+x_{n} R$ be a finitely presented module, and $P=$ $x_{1} R+\cdots+x_{n-1} R$. Then $N / P$ is finitely presented cyclic by [2, Ex.17, P233]. If $\operatorname{Pd}(N)=0$, then $P$ is f.g. torsionfree, and hence projective by Proposition 2.3.5. If $P d(N)=1$, then $P$ is a finitely presented module by Proposition 4.1.1. Therefore $P$ is a finitely presented module with $n-1$ generators. Thus, the induction hypothesis implies that there is a chain of submodules of $P$ :

$$
0=P_{0} \subset P_{1} \subset \cdots \subset P_{k}=P
$$

such that $P_{i+1} / P_{i}$ are finitely presented cyclic for all $i=0,1, \cdots, k-1$. Hence

$$
0=P_{0} \subset P_{1} \subset \cdots \subset P_{k}=P \subseteq N
$$

is the required chain for $N$

\subsection{Divisible modules of projective dimension at most one}

Given a Prüfer ring $R$, we construct a special divisible module $\partial$ with projective dimension at most one by following Fuchs, and then we characterize all divisible right $R$-modules with projective dimension at most one by using 
the module $\partial$.

Lemma 4.2.1 [Fuchs]. Let $0=M_{0} \subseteq M_{1} \subseteq \cdots \subseteq M_{\alpha} \subseteq \cdots(\alpha<\rho)$ be a well-ordered continuous ascending chain of submodules of $M_{R}$. Suppose that $\operatorname{Ext}_{R}^{1}\left(M_{\alpha+1} / M_{\alpha}, X\right)=0$ for all $\alpha+1<\rho$, and some $X \in M o d-R$. Then $\operatorname{Ext}_{R}^{1}\left(\bigcup_{\alpha<\beta} M_{\alpha}, X\right)=0$ for every $\beta \leq \rho$.

Proof. We can assume $\cup M_{\alpha}=M$. Let $0 \rightarrow X \rightarrow E \rightarrow M \rightarrow 0$ be an extension of $X$ by $M$. We want to show that it splits by constructing a module $A$ such that $E=X \oplus A$.

Let $0 \rightarrow X \rightarrow E_{\alpha} \rightarrow M_{\alpha} \rightarrow 0$ be the exact sequence induced by the inclusion $M_{\alpha} \rightarrow M$. Obviously, this splits for $\alpha=0$. Regard $E$ as the union of the ascending chain

$$
0=E_{0} \subseteq E_{1} \subseteq \cdots \subseteq E_{\alpha} \subseteq \cdots(\alpha<\rho),
$$

and suppose that we have found $R$-submodules $A_{\beta}$ of $E_{\beta}$ for each $\beta<\alpha$ such that

$$
0=A_{0} \subseteq A_{1} \subseteq \cdots \subseteq A_{\beta} \subseteq \cdots(\beta<\alpha),
$$

is a well-ordered continuous ascending chain satisfying $E_{\beta}=X \oplus A_{\beta}(\beta<\alpha)$. If $\alpha$ is a limit ordinal, then set $A_{\alpha}=\cup_{\beta<\alpha} A_{\beta}$. This will satisfy $E_{\alpha}=$ $X \oplus A_{\alpha}$. If $\alpha-1$ exists, then $E_{\alpha} / A_{\alpha-1}$ is an extension of $E_{\alpha-1} / A_{\alpha-1} \cong X$ by $E_{\alpha} / E_{\alpha-1} \cong M_{\alpha} / M_{\alpha-1}$. By our hypothesis, this splits, i.e., $E_{\alpha} / A_{\alpha-1}=$ $\left(E_{\alpha-1} / A_{\alpha-1}\right) \oplus\left(A_{\alpha} / A_{\alpha-1}\right)$ for some $A_{\alpha} \supseteq A_{\alpha-1}$. Evidently, $E_{\alpha}=X+A_{\alpha}$. On the other hand, $X \cap A_{\alpha}=X \cap E_{\alpha-1} \cap A_{\alpha}=X \cap A_{\alpha-1}=0$, thus $E_{\alpha}=X \oplus A_{\alpha}$. Therefore, there is a well-ordered continuous ascending chain

$$
0=A_{0} \subseteq A_{1} \subseteq \cdots \subseteq A_{\alpha} \subseteq \cdots(\alpha<\rho)
$$


such that $E_{\alpha}=X \oplus A_{\alpha}$ for all $\alpha<\rho$. Set $A=\cup_{\alpha<\rho} A_{\alpha}$. Then $E=X \oplus A$.

The module $\partial$ was first constructed by Fuchs. Facchini used a slight modification of $\partial$ to study divisible modules over a commutative domain [7]. Here, we follow Facchini for the construction of $\partial$.

Given a ring $R$, for every positive integer $k$ let

$$
X_{k}=\left\{\left(r_{1}, \cdots, r_{k}\right): r_{i} \in \mathcal{C}_{R}(0), i=1, \ldots, k\right\} \text { and } X_{0}=\{w\} .
$$

Set $X=\bigcup_{j \geq 0} X_{j}$. For $\left(r_{1}, \cdots, r_{k}\right),\left(r_{1}^{\prime}, \cdots, r_{l}^{\prime}\right)$, both in $X$, we define

$$
\left(r_{1}, \cdots, r_{k}\right)=\left(r_{1}^{\prime}, \cdots, r_{l}^{\prime}\right) \Leftrightarrow k=l \text { and } r_{i}=r_{i}^{\prime} \text { for } i=1, \cdots, k \text {. }
$$

Let $U$ be the free right $R$-module with basis $X$, i.e.,

$$
U=w R \oplus\left[\oplus\left(r_{1}\right) \in X_{1}\left(r_{1}\right) R\right] \oplus\left[\oplus\left(r_{1}, r_{2}\right) \in X_{2}\left(r_{1}, r_{2}\right) R\right] \oplus \cdots .
$$

Set

$$
Y=\left\{\left(r_{1}, \cdots, r_{k}\right) r_{k}-\left(r_{1}, \cdots, r_{k-1}\right):\left(r_{1}, \cdots, r_{k}\right) \in X_{k}, k>0\right\}
$$

(note $\left(r_{1}, \cdots, r_{k-1}\right)=w$ if $k=1$ ), and let $V$ be the submodule of $U$ generated by $Y$. We define $\partial=U / V$.

An element $a$ of $R$ is called left invertible if $a b=1$ for some $b \in R$. And such a $b$ is called a right inverse of $a$. Some basic facts about $\partial$ are included in the following proposition.

Proposition 4.2.1 Let $\partial_{k}$ be the submodule of $\partial$ generated by $\{\xi+V: \xi \in$ $\left.\bigcup_{i \leq k} X_{i}\right\}$. Then

(a) $0 \subset \partial_{0} \subseteq \partial_{1} \subseteq \cdots \subseteq \partial_{k} \subseteq \cdots$, and $\partial=\bigcup_{k \geq 0} \partial_{k}$. If every element in $\mathcal{C}_{R}(0)$ is left invertible, then $\partial=\partial_{0}$; if some element in $\mathcal{C}_{R}(0)$ is not left invertible, then $\partial_{k} \subset \partial_{k+1}$ for all $k$;

(b) $\partial_{0}=\bar{w} R(\bar{w}=w+V) \cong R_{R}$; And $\partial / \partial_{0}$ is torsion if $R$ is a right order; 
(c) For each $k \geq 0$, either $\partial_{k+1} / \partial_{k} \neq 0$, or there exists a non-empty subset $Z_{k}$ of $X_{k+1}$ such that $\partial_{k+1} / \partial_{k}=\bigoplus_{\xi \in Z_{k}}\left(\bar{\xi}+\partial_{k}\right) R$ with $\left(\bar{\xi}+\partial_{k}\right)^{\perp}=r_{k+1} R$, where $\bar{\xi}=\xi+V$ and $\xi=\left(r_{1}, \cdots, r_{k+1}\right)$;

(d) $P d\left(\partial_{k+1} / \partial_{k}\right) \leq 1$ for every $k \geq 0$, and $P d(\partial) \leq 1$;

(e) $A$ module $D_{R}$ is called divisible if $D r=D$ for every $r \in \mathcal{C}_{R}(0)$. Let $D_{R}$ be a divisible module, and $a \in D$. Then there exists a homomorphism $f: \partial \longrightarrow D$ with $f(\bar{w})=a$;

(f) If the ring $R$ is an order, then $\partial$ is divisible;

(g) Let the ring $R$ be an order. For every divisible module $M_{R}$, there exists an exact sequence $0 \rightarrow N \rightarrow D \rightarrow M \rightarrow 0$ of divisible right $R$-modules such that $D$ is a direct sum of modules each of which is isomorphic to $\partial / \bar{w} r_{i} R$ for some $r_{i} \in \mathcal{C}_{R}(0) \cup\{0\}$; if $M$ is divisible torsion then we can choose every such $r_{i}$ in $\mathcal{C}_{R}(0)$.

Proof. (a). Directly from the constructions of $\partial$ and $\partial_{k}$, we have $0 \subset \partial_{0} \subseteq$ $\partial_{1} \subseteq \cdots \subseteq \partial_{k} \subseteq \cdots$, and $\partial=\bigcup_{k \geq 0} \partial_{k}$. Moreover, $w \notin V$ implies that $\partial_{0} \neq 0$. If $r \in \mathcal{C}_{R}(0)$ is not left invertible, then, for each $k,\left(r_{1}, \cdots, r_{k}\right)+V \in \partial_{k} \backslash \partial_{k-1}$, where $r_{1}=\cdots=r_{k}=r$. If every element in $\mathcal{C}_{R}(0)$ is left invertible, then for each $\left(r_{1}, \cdots, r_{k}\right) \in X_{k},\left(r_{1}, \cdots, r_{k}\right)+V=\left(\left(r_{1}, \cdots, r_{k-1}\right)+V\right) s_{k} \in \partial_{k-1}$, where $s_{k}$ is a right inverse of $r_{k}$. It follows that $\partial_{k}=\partial_{k-1}=\cdots$.

(b). For any $0 \neq a \in R, w a \notin V$. This implies that $\partial_{0}=\bar{w} R$ is a free $R$-module with a single element basis set $\{\bar{w}\}$. So $\partial_{0} \cong R_{R}$. Since $R$ is a right order, $\tau\left(\partial / \partial_{0}\right)$ is a submodule. From the construction of $\partial$, we see $\tau\left(\partial / \partial_{0}\right)$ contains a set of generators of $\partial / \partial_{0}$. It follows that $\partial / \partial_{0}=\tau\left(\partial / \partial_{0}\right)$. 
(c). If $\partial_{k+1} / \partial_{k} \neq 0$, then, by $(a), \mathcal{C}_{R}(0)$ contains an element which is not left invertible. Set $Z_{k}=\left\{\left(r_{1}, \cdots, r_{k+1}\right) \in X_{k+1}: r_{k+1}\right.$ is not left invertible \}. Note that if $\xi=\left(r_{1}, \cdots, r_{k+1}\right) \in X_{k+1}$ and $r_{k+1}$ is left invertible, then $\bar{\xi} \in \partial_{k}$. Thus $\bar{\xi}+\partial_{k}=0$. From the constructions of $\partial, \partial_{k}$, and $\partial_{k+1}$, we have $\partial_{k+1} / \partial_{k}=\bigoplus_{\xi \in Z_{k}}\left(\bar{\xi}+\partial_{k}\right) R$, and for each $\xi=\left(r_{1}, \cdots, r_{r_{k+1}}\right) \in Z_{k}$, $\left(\bar{\xi}+\partial_{k}\right)^{\perp}=r_{k+1} R$.

$(d)$. By $(c), \partial_{k+1} / \partial_{k}=\bigoplus_{\xi \in Z_{k} \subseteq X_{k+1}}\left(\bar{\xi}+\partial_{k}\right) R$. By defining a well-ordering on $Z_{k}$, we can write $\partial_{k+1} / \partial_{k}$ as the union of a well-ordered continuous chain of submodules with each factor of the chain isomorphic to some $\left(\vec{\xi}+\partial_{k}\right) R$. Since $0 \rightarrow r_{k+1} R \rightarrow R \rightarrow\left(\bar{\xi}+\partial_{k}\right) R \rightarrow 0$ is exact for $\xi=\left(r_{1}, \cdots, r_{k+1}\right) \in Z_{k}$, we have $\operatorname{Pd}\left(\partial_{k+1} / \partial_{k}\right) \leq 1$ by Lemma 4.1.2. Therefore, by Lemma 4.1.2, $\operatorname{Pd}(\partial) \leq 1$.

(e). We construct a map $\eta: \cup_{0 \leq k} X_{k} \rightarrow D$ as follows:

Let $\eta(w)=a$. For $(r) \in X_{1}$, choose one $x \in D$ with $x r=a$ and let $\eta((r))=x$. Suppose for each element $\xi$ of $X_{k-1}, \eta(\xi)$ has been defined. For $\left(r_{1}, \cdots, r_{k}\right) \in X_{k}$, we choose one $x \in D$ with $x r_{k}=\eta\left(\left(r_{1}, \cdots, r_{k-1}\right)\right)$ and let $\eta\left(\left(r_{1}, \cdots, r_{k}\right)=x\right.$. In this manner, we define a map $\eta: \cup_{0 \leq k} X_{k} \longrightarrow D$. Since $U$ is a free $R$-module with a basis $\cup_{0 \leq k} X_{k}$, the map $\eta$ determines uniquely a homomorphism $\eta: U \rightarrow D$. From the construction of $\eta$, we see $Y \subseteq \operatorname{Ker}(\eta)$, and so $V \subseteq \operatorname{Ker}(\eta)$. Therefore there is a natural epimorphism $\partial=U / V \stackrel{\phi}{\rightarrow} U / \operatorname{Ker}(\eta)$. $\eta$ induces a monomorphism $U / \operatorname{Ker}(\eta) \stackrel{\bar{\eta}}{\rightarrow} D$. Then $\partial \stackrel{\bar{\eta} \circ \phi}{\rightarrow} D$ is a homomorphism such that $(\bar{\eta} \circ \phi)(\bar{w})=a$.

$(f) . \quad$ Let $Q=Q_{c l}(R), \xi=\sum_{i, k}\left(\left(r_{1 k}^{i}, r_{2 k}^{i}, \cdots, r_{k k}^{i}\right)+V\right) a_{i k} \in \partial$, and $t \in \mathcal{C}_{R}(0)$. Then $t a_{i k} t^{-1} \in Q$. Write $t a_{i k} t^{-1}=p_{i k}^{-1} q_{i k}$ for some $q_{i k} \in R$ and some $p_{i k} \in \mathcal{C}_{R}(0)$. By [18, Lemma 5.1, P87], $p_{i k}^{-1}=r^{-1} a_{i}$ for some $a_{i} \in R$ 
and $r \in \mathcal{C}_{R}(0)$. Then $t a_{i k} t^{-1}=r^{-1} a_{i} q_{i k}$, and thus $a_{i} q_{i k} t=r t a_{i k}$. Therefore $\xi=\sum_{i, k}\left(\left(r_{1 k}^{i}, \cdots, r_{k k}^{i}\right)+V\right) a_{i k}=\sum_{i, k}\left(\left(r_{1 k}^{i}, \cdots, r_{k k}^{i}, t, r\right)+V\right) r t a_{i k}=$ $\left[\sum_{i, k}\left(\left(r_{1 k}^{i}, \cdots, r_{k k}^{i}, t, r\right)+V\right) a_{i} q_{i k}\right] t$.

$(g)$. Given a divisible module $M_{R}$. For any nonzero element $a$ in $M$, if $a r \neq 0$ for any $r \in \mathcal{C}_{R}(0)$, then we let $I_{a}=\{(a, 0)\}$; otherwise, we set $I_{a}=\left\{(a, r): r \in \mathcal{C}_{R}(0)\right.$ with $\left.a r=0\right\}$. For each $(a, r) \in I_{a}$, we choose an $f_{a, r} \in \operatorname{Hom}(\partial / \bar{w} r R, M)$ satisfying $f_{a, r}(\bar{w}+\bar{w} r R)=a$. Such an $f_{a, r}$ exists by $(e)$. Let $D=\oplus_{a} \oplus_{r \in I_{a}}(\partial / \bar{w} r R)$. Then $\left\{f_{a, r}\right\}$ induces a homomorphism $f=\oplus f_{a, r}: D \longrightarrow M$, and $f$ is clearly onto. Also, $f$ induces an exact sequence:

$$
0 \longrightarrow N \longrightarrow D \longrightarrow M \longrightarrow 0, \quad \text { where } N=\operatorname{ker}(f)
$$

By $(f), D$ is divisible. To see $N$ is divisible, let $x \in N$ and $t \in \mathcal{C}_{R}(0)$. Since $D$ is divisible, $x=y t$ for some $y \in D$. Let $z=f(y) \in M$. Then $z t=f(y) t=f(y t)=f(x)=0$. Therefore the map $g: R / t R \rightarrow M$ defined by $g(\bar{b})=z b$ is a well-defined homomorphism. Define $h: R \rightarrow D$ by $h(b)=(\bar{w}+\bar{w} t R) b$. Then $h(t)=0$, and thus $h$ induces a homomorphism $\bar{h}: R / t R \rightarrow D$. Directly from the definition of $D$ and the map $f$, we have $f \circ h=g$. Let $u=y-\bar{h}(\overline{1}) \in D$. Then $f(u)=f(y)-f \circ \bar{h}(\overline{1})=z-g(\overline{1})=0$, and $u t=y t-\bar{h}(\overline{1}) t=x$. Therefore $N$ is divisible. The last part of $(g)$ is now clear from the proof above.

A short exact sequence of right $R$-modules: $0 \rightarrow M^{\prime} \rightarrow M \rightarrow M^{\prime \prime} \rightarrow 0$ is called pure if $M^{\prime} \otimes_{R} L \longrightarrow M \otimes_{R} L$ is a monomorphism for every left $R$ module $L$. A module $N_{R}$ is called absolutely pure (or FP-injective) if every exact sequence $0 \rightarrow N_{R} \rightarrow M_{R} \rightarrow P_{R} \rightarrow 0$ is pure. 
Proposition 4.2.2 Let $M_{R}$ be a module over a Prüfer ring $R$. Then the following are equivalent:

(a) $M_{R}$ is divisible;

(b) $\operatorname{Ext}_{R}^{1}(R / r R, M)=0$, for every $r \in \mathcal{C}_{R}(0)$;

(c) $\operatorname{Ext}_{R}^{1}(R / I, M)=0$, for every f.g. right ideal I of $R$;

(d) $M_{R}$ is absolutely pure.

Proof. $(a) \Leftrightarrow(b)$. From the exact sequence $0 \rightarrow r R \rightarrow R \rightarrow R / r R \rightarrow 0$, we have an exact sequence $\operatorname{Hom}(R, M) \rightarrow \operatorname{Hom}(r R, M) \rightarrow \operatorname{Ext}^{1}(R / r R, M) \rightarrow$ 0 . Therefore, $\operatorname{Ext}^{1}(R / r R, M)=0$ if and only if for every homomorphism $r R \stackrel{f}{\rightarrow} M$, there exists a homomorphism $R \stackrel{g}{\rightarrow} M$ such that $g$ extends $f$. If $M_{R}$ is divisible, $r \in \mathcal{C}_{R}(0)$ and $r R \stackrel{f}{\rightarrow} M$ is a homomorphism, then $f(r)=y r$ for some $y \in M$. Define $g: R \rightarrow M$ by $g(1)=y$. Then $g$ extends $f$, and so $\operatorname{Ext}^{1}(R / r R, M)=0$. Conversely, let $x \in M, r \in \mathcal{C}_{R}(0)$. Clearly $f: r R \rightarrow M$ via $f(r a)=r x$ is a homomorphism. Since $f$ can be extended to a homomorphism $R \stackrel{g}{\rightarrow} M$, then $x=r g(1)$. Therefore $D$ is divisible.

$(b) \Leftarrow(c)$. Trivial.

$(b) \Rightarrow(c)$. Let $I$ be a f.g. right ideal of $R$. From the exact sequence $0 \rightarrow$ $I_{R} \stackrel{i}{\rightarrow} R_{R} \rightarrow(R / I)_{R} \rightarrow 0$, we have the exact sequence $\operatorname{Hom}(R, M) \stackrel{\operatorname{Hom}(i, M)}{\longrightarrow}$ $\operatorname{Hom}(I, M) \longrightarrow \operatorname{Ext}_{R}^{1}(R / I, M) \longrightarrow 0$. Therefore $\operatorname{Ext}_{R}^{1}(R / I, M)=0$ if and only if $\operatorname{Hom}(i, M)$ is onto if and only if each homomorphism $f: I_{R} \rightarrow M$ can be extended to $R$. We can find a right ideal $J$ of $R$ which is maximal with respect to $I \cap J=0$. Then $I+J=I \oplus J$ is an essential right ideal of $R$. By Proposition $1.2, I+J$ contains an element $r \in \mathcal{C}_{R}(0)$. Write $r=r_{1}+r_{2}$, for some $r_{1} \in I$ and some $r_{2} \in J$, and let $K=I+r_{2} R=I \oplus r_{2} R$. Obviously 
$f: I \rightarrow M$ can be extended to $\bar{f}: K \rightarrow M . K$ is a f.g. right ideal of $R$, hence $K$ is projective, since $R$ is semihereditary. By Proposition 1.4, there exist $\left\{a_{i}\right\} \subseteq K$ and $\left\{f_{i}\right\} \subseteq \operatorname{Hom}(K, R)$, such that for any $a \in K, f_{i}(x)=0$ for all but a finite number of the $f_{i}$, and $a=\Sigma a_{i} f_{i}(a)$. Since $K \cap \mathcal{C}_{R}(0) \neq 0$, there exists, for each $i$, a $q_{i} \in Q_{c l}(R)$ satisfying $q_{i} K \subseteq R$ such that $f_{i}(a)=q_{i} a$ for all $a \in K$. For $s \in K \cap \mathcal{C}_{R}(0)$, we have $s=\Sigma a_{i} f_{i}(s)=\left(\Sigma a_{i} q_{i}\right) s$. This implies that $\Sigma a_{i} q_{i}=1$. Since $R$ is also a left order in $Q_{c l}(R)$, there exists $t \in \mathcal{C}_{R}(0)$ such that all $t q_{i} \in R$. Now the divisibility of $M$ implies that we can write $f\left(a_{i}\right)=x_{i}$ t with all $x_{i} \in M$. Then for any $a \in K$ we obtain $f(a)=f\left(\Sigma a_{i} q_{i} a\right)=\Sigma f\left(a_{i}\right)\left(q_{i} a\right)=\Sigma x_{i}\left(t q_{i}\right) a=x a$ with $x=\Sigma x_{i} t q_{i} \in M$. Hence the map $a \mapsto x a$ from $R$ to $M$ is a $R$-homomorphism that extends $\bar{f}$.

$(c) \Leftrightarrow(d)$. Megibben and Stenström proved, independently, that $(c) \Leftrightarrow$ (d) for an arbitrary ring $R$ (see [30, Prop.1] or [35, Prop.2.6]).

The concept of a semicompact module was defined by Matlis in [28], where it was shown that a module over a commutative Prüfer domain is injective if and only if it is divisible and semicompact. The same result holds in a noncommutative Prüfer ring.

For a module $M_{R}$, let $R(M)$ denote the set of subsets of $M$ of the form $\{x \in M: x I=0\}$ for a right ideal $I$ of $R$. $M$ will be called semicompact if every finitely solvable set of congruences

$$
x \equiv x_{\alpha} \quad\left(\bmod M_{\alpha}\right)
$$

where $x_{\alpha} \in M$ and $M_{\alpha} \in R(M)$, has a solution in $M$ [28]. If we note a result of Stenström [35, Prop.2.5] that an absolutely pure module is injective if and only if it is semicompact, then the following is immediate: 
Corollary 4.2.1 Let $M_{R}$ be a module over a Prüfer ring $R$. Then $M$ is injective if and only if it is divisible and semicompact.

Proposition 4.2.3 Let $M_{R}$ be a module over a Prüfer ring $R$. If $\operatorname{Pd}(M)=$ $m \geq 1$, then $\operatorname{Ext}_{R}^{m}(M, D)=0$ for all divisible module $D_{R}$.

Proof. We induct on $m$. If $m=1$, then, by Theorem 4.1.2, $M_{R}$ is the union of a well-ordered continuous chain of submodules:

$$
0=M_{0} \subset M_{1} \subset \cdots \subset M_{\alpha} \subset \cdots \subset M_{\rho}=M \quad(\alpha<\rho)
$$

such that $M_{\alpha+1} / M_{\alpha}$ is finitely presented cyclic for all $\alpha<\rho$. Thus, for each $\alpha<\rho, M_{\alpha+1} / M_{\alpha} \cong R / I_{\alpha}$ for some f.g. right ideal $I_{R}$. Since $D_{R}$ is divisible, Proposition 4.2.2 implies that $\operatorname{Ext}_{R}^{1}\left(M_{\alpha+1} / M_{\alpha}, D\right)=0$, for every $\alpha<\rho$. By Lemma 4.2.1, $\operatorname{Ext}_{R}^{1}(M, D)=0$.

For $m>1$, let $0 \rightarrow N_{R} \rightarrow F_{R} \rightarrow M_{R} \rightarrow 0$ be an exact sequence with $F$ projective. Then $\operatorname{Pd}(N)=\operatorname{Pd}(M)-1=m-1$. Now the induction hypothesis implies that $\operatorname{Ext}_{R}^{m-1}(N, D)=0$ for all divisible module $D_{R}$. From the exact sequence $0 \rightarrow N \rightarrow F \rightarrow M \rightarrow 0$, we have $\operatorname{Ext}_{R}^{k}(N, D) \cong \operatorname{Ext}_{R}^{k+1}(M, D)$ for all $k \geq 1$. Therefore $\operatorname{Ext}_{R}^{m}(M, D)=0$ for every divisible module $D_{R}$.

Remark 4.2.1 Proposition 4.2.3 generalizes a result of L.Fuchs [13, Prop.3.9, P126].

We now can give the following characterization of divisible modules of projective dimension at most one:

Proposition 4.2.4 Let $M_{R}$ be a module over a Prüfer ring $R$. Then $M$ is divisible with $\operatorname{Pd}(M) \leq 1$ if and only if it is a summand of a direct sum of modules of the form $\partial / \bar{w} r_{i} R$, where every $r_{i} \in \mathcal{C}_{R}(0) \cup\{0\}$. 
Proof. $(\Rightarrow)$. By Proposition 4.2.1 $(g)$, there exists an exact sequence $0 \rightarrow$ $N \rightarrow D \rightarrow M \rightarrow 0$, where $N$ is divisible, and $D \cong \bigoplus_{r \in S} \partial / \bar{w} r R$ for a subset $S$ of $\mathcal{C}_{R}(0) \cup\{0\}$. If $\operatorname{Pd}(M)=0$, then $\operatorname{Ext}_{R}^{1}(M, N)=0$ from the definition of projective dimension. If $\operatorname{Pd}(M)=1$, then Proposition 4.2.3 implies $\operatorname{Ext}_{R}^{1}(M, N)=0$. Hence, $0 \rightarrow N \rightarrow D \rightarrow M \rightarrow 0$ splits. It follows that $M$ is a summand of $D$.

$(\Leftarrow)$. Let $D$ be as above, and $M$ be a summand of $D$. Then Proposition 4.2.1 $(f)$ implies $M$ is divisible. We know $P d(\partial) \leq 1$ from $(d)$ of Proposition 4.2.1. Suppose $P d(\partial)=1$, we have $P d(\partial / \bar{w} r R) \leq 1$ for all $r \in R$ by Proposition 4.1.1. Then, a similar proof of Proposition 4.2.1 (d) shows that $\operatorname{Pd}(D) \leq 1$. If $\operatorname{Pd}(D)=1$, then we have $\operatorname{Pd}(M)=1$ by Proposition 4.1.1. On the other hand, $P d(D)=0$ implies $M$ is projective and hence $P d(M)=0$. Therefore $P d(M) \leq 1$ holds if $P d(\partial)=1$. Suppose $P d(\partial)=0$, i.e., $\partial$ is projective, then $\partial$ is torsionfree. Therefore $\bar{w} r R$ is f.g. torsionfree. It follows from Proposition 2.3.5 that $\bar{w} r R$ is projective. Therefore we still have $\operatorname{Pd}(\partial / \bar{w} r R) \leq 1$. Repeating the argument above, we have $\operatorname{Pd}(M) \leq 1$.

Corollary 4.2.2 Let $M_{R}$ be a module over a Prüfer ring $R$. Then $M_{R}$ is divisible torsion with $P d(M) \leq 1$ if and only if it is a summand of a direct sum of modules of the form $\partial / \bar{w} r_{i} R$, where each $r_{i} \in \mathcal{C}_{R}(0)$.

Proof. It follows from the last part of $(g)$ of Proposition 4.2.1 and the proof of Proposition 4.2.4.

Let $C(R)$ denote the center of a ring $R$, and $r \in \mathcal{C}_{R}(0)$. Suppose $1 \neq s \in$ $C(R) \cap \mathcal{C}_{R}(0)$. We define two maps as follows: 


$$
\phi: \cup_{0 \leq k} X_{k} \longrightarrow \partial
$$

by $\phi(w)=\overline{(r)}-\overline{(r s)} s$, and

And

$$
\phi\left(\left(r_{1}, \cdots, r_{k}\right)\right)=\overline{\left(r, r_{1}, \cdots, r_{k}\right)}-\overline{\left(r s, r_{1}, \cdots, r_{k}\right)} s \text { for } k \geq 1 .
$$

$$
\psi: \cup_{0 \leq k} X_{k} \longrightarrow \partial / \bar{w} r R
$$

by $\psi(w)=\overline{0}, \psi((r))=\bar{w}+\bar{w} r R$, and

$$
\psi\left(\left(r_{1}, r_{2}, \cdots, r_{k}\right)\right)=\overline{\left(r_{2}, \cdots, r_{k}\right)}+\bar{w} r R \text { if } r_{1}=r \text {; or } \overline{0} \text { if } r_{1} \neq r .
$$

Then $\phi$ determines uniquely a homomorphism $U \stackrel{\phi}{\rightarrow} \partial$, and $\psi$ defines a homomorphism $U \stackrel{\psi}{\rightarrow} \partial / \bar{w} r R$. It is straightforward to check that $Y+w r R \subseteq$ $\operatorname{Ker}(\phi)$ and $V \subseteq \operatorname{Ker}(\psi)$. Therefore $\phi$ and $\psi$ induce canonically two homomorphisms

Note that

$$
U /(w r R+V) \stackrel{\Phi}{\rightarrow} U / K \operatorname{Ker}(\phi) \text { and } U / V \stackrel{\Psi}{\rightarrow} U / \operatorname{Ker}(\psi) .
$$

$$
\partial / \bar{w} r R \cong U /(w r R+V) \text { and } \partial=U / V .
$$

Then the homomorphism

$$
\Phi: \partial / \bar{w} r R \rightarrow \partial
$$

satisfies

$$
\Phi(\bar{w}+\bar{w} r R)=\overline{(r)}-\overline{(r s)} s
$$

and

$$
\Phi\left(\overline{\left(r_{1}, \cdots, r_{k}\right)}+\bar{w} r R\right)=\overline{\left(r, r_{1}, \cdots, r_{k}\right)}-\overline{\left(r s, r_{1}, \cdots, r_{k}\right)} s \text { for } k \geq 1 ;
$$

and the homomorphism

$$
\Psi: \partial \rightarrow \partial / \bar{w} r R
$$

satisfies

and

$$
\Psi(\bar{w})=\overline{0}, \Psi((\bar{r}))=\bar{w}+\bar{w} r R
$$




$$
\Psi\left(\overline{\left(r, r_{2}, \cdots, r_{k}\right)}\right)=\overline{\left(r_{2}, \cdots, r_{k}\right)}+\bar{w} r R
$$

and

$$
\Psi\left(\overline{\left(r_{1}, r_{2}, \cdots, r_{k}\right)}\right)=\overline{0} \text { if } r_{1} \neq r
$$

Let $\partial \stackrel{n}{\rightarrow} \partial / \partial_{0}$ be the natural homomorphism, and $\Phi_{1}=n \circ \Phi$. Since $\Psi(\bar{w})=$ $\overline{0}, \Psi$ induces a homomorphism $\Psi_{1}: \partial / \partial_{0} \rightarrow \partial / \bar{w} r R$.

Lemma 4.2.2 Let $\Phi, \Psi, \Phi_{1}$, and $\Psi_{1}$ be the same as above.

(a) $\Psi \circ \Phi=1_{\partial / \bar{w} r R}$. In particular, $\partial / \bar{w} r R$ is a summand of $\partial$;

(b) $\Psi_{1} \circ \Phi_{1}=1_{\partial / \bar{w} r R}$. In particular, $\partial / \bar{w} r R$ is a summand of $\partial / \partial_{0}$.

Proof. (a). Since $Z=\left\{\bar{\xi}+\bar{w} r R: \xi \in \cup X_{k}\right\}$ is a set of generators of $\partial / \bar{w} r R$, it suffices to check that $\Psi \circ \Phi$ fixs every element of $Z$. However, the verification is straightforward.

(b). Similarly.

Theorem 4.2.1 If $R$ is a Prüfer ring, and $C(R) \neq\{0,1\}$ (e.g., if the characteristic of $R \neq 2$ ), then $M_{R}$ is divisible with $P d(M) \leq 1$ if and only if it is a summand of a direct sum of copies of $\partial$.

Proof. Note that if $R$ is a Prüfer ring, then $C(R) \subseteq \mathcal{C}_{R}(0)$. Now apply Proposition 4.2.4 and Lemma 4.2.2.

Theorem 4.2.2 If $R$ is a Prüfer ring, and $C(R) \neq\{0,1\}$, then $M_{R}$ is divisible torsion with $\operatorname{Pd}(M) \leq 1$ if and only if it is a summand of a direct sum of copies of $\partial / \partial_{0}$.

Proof. By Corollary 4.2.2, and Lemma 4.2.2. 


\section{References}

[1] J.H.Alajbegovic and N.I.Dubrovin, Noncommutative Prüfer rings, J. Algebra 135, 165-176, 1990.

[2] F.W.Anderson and K.R.Fuller, Rings and Categories of Modules, Springer-Verlag, New York/Heidelberg/Berlin, 1974.

[3] K.Asano, Arithmetische Idealtheorie in nichtkommutativen Ringen, Japanese J. Math. 16, 1-36, 1939.

[4] H.Bass, Finitistic dimension and homological generalization of semiprimary rings, Trans. Amer. Math. Soc. 19, 466-488, 1960.

[5] H.H.Brungs, Generalized discrete valuation rings, Can. J. Math. 21, 1404-1408, 1969.

[6] S.U.Chase, A generalization of the ring of triangular matrices, Nagoya Math. J. 18, 13-25, 1961.

[7] A.Facchini, Divisible modules over integral domains, Ark. Mat 26 (1), $67-85,1988$.

[8] C.Faith, Algebra: Rings, Modules and Categories, Springer-Verlag, Berlin/Heidelberg, 1973.

[9] C.Faith, Algebra II: Ring Theory, Springer-Verlag, Berlin/ Heidelberg, 1976. 
[10] C.Faith and S.Page, FPF Ring Theory: Faithful Modules and Generators of Mod-R, London Math.Soc. Lectures Note Series, Cambridge Univ. Press, Cambridge/New York, 1984.

[11] L.Fuchs, On projective dimensions of modules over valuation domains, Abelian Group Theory, Lecture Notes in Math. 1006, Springer, 589-598, 1983.

[12] L.Fuchs, On divisible modules over domains, Proceedings of Conference on Abelian Groups and Modules in Udine, 1984.

[13] L.Fuchs and L.Salce, Modules over Valuation Domains, Lecture Notes in Pure and Applied Mathematics, 97, Marcel Dekker,1985.

[14] E.R.Gentile, On rings with one-sided field of quotients, Proc. Amer. Math. Soc. 11, 380-384, 1960.

[15] A.W.Goldie, The structure of prime rings under ascending chain conditions, Proc. London Math. Soc. 8 (3), 589-608, 1958.

[16] A.W.Goldie, Semi-prime rings with maximum conditions, Proc. London Math. Soc. 10 (3), 201-220, 1960.

[17] K.R.Goodearl, Ring Theory: Nonsingular Rings and Modules, Marcel Dekker, Inc., 1976.

[18] K.R.Goodearl and R.B.Warfield Jr, An Introduction to Noncommutative Noetherian Rings, London Math. Soc., Cambridge Univ. Press, 1989. 
[19] N.Jacobson, Basic Algebra II, San Francisco Freeman, 1980.

[20] S.K.Jain and S.R.López-Permouth, Rings whose cyclics are essentially embeddable in projective modules, J. Alg. 128 (1), 257-269, 1990.

[21] S.K.Jain, S.R.López-Permouth and S.Singh, On a class of QI rings, Glasgow Math. J. 34, 75-81, 1992.

[22] J.P.Jans, Rings and Homology, Holt, Rinehart and Winston 1964.

[23] A.V.Jategaunkar, Localization in Noetherian Rings, Cambridge University Press, Cambridge, London, New York, 1986.

[24] J.Lambek, Rings and Modules, Blaisdell, New York, corrected reprint Chelsea, $1966 ; 1976$.

[25] L.Levy, Torsionfree and divisible modules over non-integral domains, Canad. J. Math. 15, 132-151, 1963.

[26] S.R.López-Permouth, S.T.Rizvi and M.F.Yousif, Some characterizations of semiprime Goldie rings, preprint (1991).

[27] H.Marubayashi, Noncommutative Krull rings, Osaka J. Math. 12, 703$714,1975$.

[28] E.Matlis, Injective module over Prüfer rings, Nagoya Math.J. 15, 57-69, 1957.

[29] J.C.McConnell and J.C.Robson, Noncommutative Noetherian Rings, Wiley-Interscience, New York, 1987. 
[30] C.Megibben, Absolutely pure modules, Proc.Amer.Math.Soc, 26, 561$566,1970$.

[31] J.D.O'Neill, An unusual ring, J. London Math. Soc. 44(2), 95-101, 1991.

[32] J.C.Robson, Noncommutative Dedekind rings, J. Algebra 9, 249-265, 1968.

[33] F.L.Sandomierski., Nonsingular rings, Proc. Amer. Math. Soc. 19, 22530, 1968.

[34] L.Small, Semihereditary rings, Bull. Amer. Math. Soc. 73, 656-658, 1967.

[35] B.Stenström., Coherent rings and FP-injective modules, J.London Math.Soc. 2(2), 323-329, 1970.

[36] B.Strenström, Rings of Quotients, Grundl.der Math. Wiss., 217 Springer-Verlag, Berlin/Heidelberg/New York 1975.

[37] H.Tachikawa, Quasi-Frobenius Rings and Generalizations of QF-3 and QF-1 Rings, Lectures Notes in Mathematics, 351 Springer/Heidelberg/New York, 1973.

[38] R.B.Warfield,Jr, Serial rings and finitely presented modules, J. Algebra 37, 187-222, 1975.

[39] Y.Zhou, Noncommutative Prüfer rings and some generalizations, Comm. in Algebra 20(9), 2609-2633, 1992. 
[40] Y.Zhou, Strongly compressible modules and semiprime right Goldie rings, Comm. in Algebra 21(2), 687-698, 1993.

[41] Y.Zhou, Modules of projective dimension one over noncommutative Prüfer rings, to appear in Comm. in Algebra, 1993/94. 